\title{
A COALGEBRAIC TAKE ON REGULAR AND $\omega$-REGULAR BEHAVIOURS
}

\author{
TOMASZ BRENGOS
}

e-mail address: t.brengos@mini.pw.edu.pl

Faculty of Mathematics and Information Science, Warsaw University of Technology, ul. Koszykowa 75, 00-662 Warszawa, Poland

\begin{abstract}
We present a general coalgebraic setting in which we define finite and infinite behaviour with Büchi acceptance condition for systems whose type is a monad. The first part of the paper is devoted to presenting a construction of a monad suitable for modelling (in)finite behaviour. The second part of the paper focuses on presenting the concepts of a (coalgebraic) automaton and its $(\omega-)$ behaviour. We end the paper with coalgebraic Kleene-type theorems for $(\omega-)$ regular input. The framework is instantiated on non-deterministic (Büchi) automata, tree automata and probabilistic automata.
\end{abstract}

\section{Contents}

1. Introduction

1.1. Motivations

1.2. The aim of the paper

2. Basic notions

2.1. Non-deterministic automata

2.2. Tree automata

2.3. Algebras and coalgebras

2.4. Monads

2.5. Coalgebras with internal moves 11

2.6. Categorical order enrichment 12

2.7. Lawvere theories 13

3. Non-deterministic automata, coalgebraically 13

3.1. Non-deterministic automata 13

3.2. Tree automata 16

3.3. Kleene theorems, categorically 16

$\begin{array}{ll}\text { 3.4. Beyond tree automata } & 17\end{array}$

Key words and phrases: bisimulation, coalgebra, epsilon transition, labelled transition system, tau transition, internal transition, logic, monad, Büchi automata, Buechi automata, saturation, weak bisimulation, infinite trace.

This work has been supported by National Centre for Research and Development Grant CYBERSECI$\mathrm{DENT} / 456962 / \mathrm{III} / \mathrm{NCBR} / 2020$. 
4. Monads for (in)finite behaviour 18

4.1. Preliminaries 18

4.2. Lifting monads to algebras 19

5. Abstract automata and their behaviour 21

5.1. Finite and infinite behaviour 22

5.2. Additional remarks 23

5.3. Kleene theorems 24

6. Probabilistic automata 33

6.1. Preliminaries 34

6.2. Choosing the right monad $\quad 35$

6.3. (In)finite behaviour $\quad 37$

6.4. Summary 42

7. Summary $\quad 42$

References $\quad 43$

\section{INTRODUCTION}

Automata theory is one of the core branches of theoretical computer science and formal language theory. One of the most fundamental state-based structures considered in the literature is a non-deterministic automaton and its relation with languages. Non-deterministic automata with a finite state-space are known to accept regular languages. These languages are characterized as subsets of words over a fixed finite alphabet that can be obtained from simple languages via a finite number of applications of three types of operations: union, concatenation and the Kleene star operation [HMRU00, Kle56]. This result is known under the name of Kleene theorem for regular languages and readily generalizes to other types of finite input (see $\quad R::=\varnothing\left|a, a \in \Sigma_{\varepsilon}\right| R+R|R \cdot R| R^{*}$ e.g. [PP04]).

On the other hand, non-deterministic au-

Figure 1: Regular expression grammar tomata have a natural infinite semantics which is given in terms of infinite input satisfying the so-called Büchi acceptance condition (or $B A C$ in short). The condition takes into account the terminal states of the automaton and requires them to be visited infinitely often. It is a common practise to use the term Büchi automata in order to refer to automata whenever their infinite semantics is taken into consideration.

Although the standard type of infinite input of a Büchi automaton is the set of infinite words over a given alphabet, other types (e.g. trees) are also commonly studied [PP04]. The class of languages of infinite words accepted by Büchi automata can also be characterized akin to the characterization of regular languages. This result is known un-

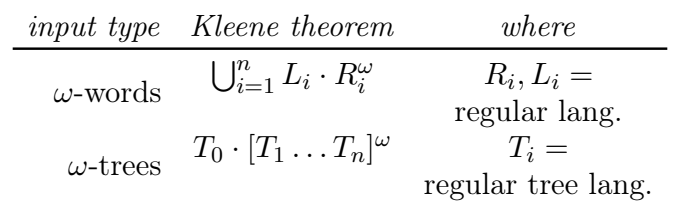

Figure 2: Kleene theorems for $\omega$-regular input der the name of Kleene theorem for $\omega$-regular languages and its variants hold for many input types (see e.g.[KN01, Büc90, GTW02, PP04]). Roughly speaking, any language recognized by a Büchi automaton can be represented in 
terms of regular languages and the infinite iteration operator $(-)^{\omega}$. This begs the question whether these systems can be placed in a unifying framework and reasoned about on a more abstract level so that the analogues of Kleene theorems for $(\omega$-)regular input are derived. The recent developments in the theory of coalgebra [CV12, SW13, USH16, Rut00] show that the coalgebraic framework may turn out to be suitable to achieve this goal.

A coalgebra $X \rightarrow F X$ is an abstract (categorical) representation of a computation of a process [Rut00, Gum99]. The coalgebraic setting has already proved itself useful in modelling finite behaviour via least fixpoints (e.g. [HJS07, SW13, Bre15]) and infinite behaviour via greatest fixpoints of suitable mappings [Jac04, Cîr10, UH15]. The infinite behaviour with $\mathrm{BAC}$ can be modelled by a combination of the two [USH16, Par81].

We plan to revisit the coalgebraic framework of (in)finite behaviour from the perspective of systems whose type functor is a monad. In the coalgebraic literature [Bre14, Bre15, BP16, BMP15, Bre18, BP19] these systems are often referred to by the name of systems with internal moves. This name is motivated by the research on a unifying theory of finite behaviour for systems with internal steps [SW13, Bre14, Bre15, BMSZ15, BP16, BMP15]. They arise in a natural manner in many branches of theoretical computer science, among which are process calculi [Mil89] (labelled transition systems and their weak bisimulation) or automata theory (automata with $\varepsilon$-moves), to name only two. Intuitively, these systems have a special computation branch that is silent. This special branch, usually labelled by the letter $\tau$ or $\varepsilon$, is allowed to take several steps and is, in some, a neutral part of the process. As thoroughly discussed in [Bre15], the nature of this type of transition suggests it is in fact (part of) the unit of a monad. Hence, from our point of view the following terms become synonymous:

$$
\text { coalgebras with internal moves }=\text { coalgebras whose type is a monad. }
$$

This observation allows for an elegant modelling of several coalgebraic behavioural equivalences which take silent steps into account [BMP15, BP16, Bre15]. If the type $T$ of a coalgebra $\alpha: X \rightarrow T X$ is a monad then the map $\alpha$ becomes an endomorphism $\alpha: X \multimap \rightarrow$ in the Kleisli category for $T$ : a natural and simple setting to study composition and fixpoints. For instance, if $T$ is taken to be the
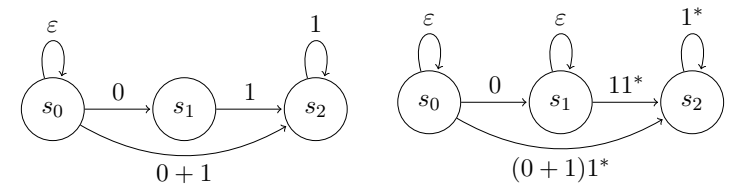

Figure 3: LTS with $\varepsilon$-moves and its saturation monad modelling labelled transition systems [Bre15] then Milner's weak bisimulation [Mil89] of an LTS given by $\alpha$ is a strong bisimulation on its saturation $\alpha^{*}$, i.e. the smallest LTS over the same state space s.t. $\alpha \leq \alpha^{*}$, id $\leq \alpha^{*}$ and $\alpha^{*} \cdot \alpha^{*} \leq \alpha^{*}$ (where the composition and the order are given in the Kleisli category for the LTS monad) [Bre15]. Hence, intuitively, $\alpha^{*}$ is the reflexive and transitive closure of $\alpha$ and is formally defined as the least fixpoint $\mu x$.(id $\vee x \cdot \alpha$ ). The fact that labelled transition systems' weak bisimulation can be modelled via saturation of endomorphisms of a given Kleisli category allows for a generalization of the setting to other systems (e.g. probabilistic [Bre15, BMP15]). The only requirement is that type functor is a monad whose Kleisli category satisfies suitable conditions for the definition of $(-)^{*}$ to be meaningful.

The reflexive and transitive closure $\alpha \mapsto \alpha^{*}$ is understood as an accumulation of a finite number of compositions of the structure with itself. Hence, the concept of coalgebraic saturation is intrinsically related to finite behaviour of systems with a monadic type. A similar treatment of infinite behaviour (and their combination used to model Büchi acceptance 
condition) in the context of coalgebras whose type is a monad has not been considered so far. The closest to this goal would be [UH15, USH16], where (in)finite trace semantics is given in the setting of $T F$-coalgebras for a monad $T$ and an endofunctor $F$. We take this treatment one step further and embed $T F$ into a monad $T F^{\infty}$ which is tailored to modelling (in)finite behaviours and their combinations. The new setting allows us to present clear definitions of coalgebraic (in)finite semantics and reason about them. In particular, it allows us to state Kleene theorems for regular and $\omega$-regular behaviours which would be challenging without the monadic types.

1.1. Motivations. Our purpose is to build a single coalgebraic setting that allows us to easily present definitions of (in)finite behaviours and reason about them aiming at their algebraic characterization. By finding a suitable monad $T$ describing the type of systems taken into consideration we are able to state generic Kleene theorems connecting syntax and semantics of languages: the former imposed by the canonical algebraic nature of $T$ and the latter given by $T$-automata and their behaviours.

By presenting a recipe to extend a functor to a suitable monad, we automatically encompass systems with invisible steps. However, this should not be viewed as our primary goal. Instead, from our point of view, it should be perceived as a by-product.

\subsection{The aim of the paper. We plan to:}

(A) revisit non-deterministic (tree) automata and their behaviour in the coalgebraic context of systems whose type is a monad,

(B) provide a type monad suitable for modelling (in)finite behaviour of general systems,

(C) present a setting for defining (in)finite behaviour for abstract automata whose type is a monad,

(D) state and prove coalgebraic Kleene theorems for $(\omega$-)regular behaviour,

(E) put probabilistic automata into the framework.

The first point is achieved in Section 3 by describing non-deterministic (tree) automata and their finite and infinite behaviour in terms of different coalgebraic (categorical) fixpoint constructions calculated in the Kleisli category for a suitable monad. Section 3 serves as a motivation for the framework presented later in Section 4 and Section 5.

Originally [Has06, SW13], coalgebras with internal moves were considered as systems $X \rightarrow T F_{\varepsilon} X$ for a monad $T$ and an endofunctor $F$, where $F_{\varepsilon} \triangleq F+\mathcal{I} d$. Under some conditions the functor $T F_{\varepsilon}$ can be embedded into the monad $T F^{*}$, where $F^{*}$ is the free monad over $F$ [Bre15]. The monad $T F^{*}$ is sufficient to model systems with internal moves and their finite behaviour [BMSZ15, Bre15, BMP15]. However, it will prove itself useless in the context of infinite behaviour. Hence, by revisiting and tweaking the construction of $T F^{*}$ from [Bre15], Section 4 gives a general description of the monad $T F^{\infty}$, the type functor $T F$ (or $T F_{\varepsilon}$ ) embeds into, which is used in the remaining part of the paper to model the combination of finite and infinite behaviour. The reason why we find the expressive power of $T F^{\infty}$ suitable is the following: the object $F^{\infty} X$ is defined for any $X$ as the carrier of the coproduct of the free algebra $F^{*} X$ over $X$ and the algebra $F^{\omega}$ obtained by inversing the final coalgebra map. Hence, by slighty abusing the notation, we can write $F^{\infty}=F^{*} \oplus F^{\omega}$.

Item (C) in the above list is achieved by using two fixpoint operators: the saturation operator $(-)^{*}$ and a new operator $(-)^{\omega}$ defined in the Kleisli category for a given monad. The combination of $(-)^{*}$ and $(-)^{\omega}$ allows us to define infinite behaviour with BAC. 
Kleene-type theorems of (D) are a direct consequence of the definitions of finite and infinite behaviour with BAC using $(-)^{*}$ and $(-)^{\omega}$.

Finally, in Section 6 we put probabilistic automata into the framework of (in)finite behaviour for systems whose type is a monad.

This paper is an extended version of [Bre18] with all missing proofs and additional Section 6 where probabilistic automata are considered.

\section{BASIC NOTIONS}

We assume the reader is familiar with basic category theory concepts like a category, a functor, an adjunction. For a thorough introduction to category theory the reader is referred to [ML78]. See also e.g. [Bre14, Bre15, BMP15] for an extensive list of notions needed here.

2.1. Non-deterministic automata. The purpose of this subsection and the next one is to recall basic definitions and properties of non-deterministic automata and their tree counterparts: an automaton, its $(\omega$-)language and Kleene theorems for regular and $\omega$-regular languages. Note that the aim of this paper is to take these notions and statements and generalize them to the categorical setting.

Classically, a non-deterministic automaton, or simply automaton, is a tuple $\mathcal{Q}=$ $\left(Q, \Sigma, \delta, q_{0}, \mathfrak{F}\right)$, where $Q$ is a finite set of states, $\Sigma$ is a finite set called alphabet, $\delta: Q \times \Sigma \rightarrow$ $\mathcal{P}(Q)$ a transition function and $\mathfrak{F} \subseteq Q$ set of accepting states. We write $q_{1} \stackrel{a}{\rightarrow} q_{2}$ if $q_{2} \in \delta\left(q_{1}, a\right)$. There are two standard types of semantics of automata: finite and infinite. The finite semantics of $\mathcal{Q}$ is defined as the set of all finite words $a_{1} \ldots a_{n} \in \Sigma^{*}$ for which there is a sequence of transitions $q_{0} \stackrel{a_{1}}{\rightarrow} q_{1} \stackrel{a_{2}}{\rightarrow} q_{2} \ldots q_{n-1} \stackrel{a_{n}}{\rightarrow} q_{n}$ which ends in an accepting state $q_{n} \in \mathfrak{F}$ [HMRU00]. The infinite semantics, also known as the $\omega$-language of $\mathcal{Q}$, is the set of infinite words $a_{1} a_{2} \ldots \in \Sigma^{\omega}$ for which there is a run $r=q_{0} \stackrel{a_{7}}{\rightarrow} q_{1} \stackrel{a_{2}}{\rightarrow} q_{2} \stackrel{a_{3}}{\rightarrow} q_{3} \ldots$ for which the set of indices $\left\{i \mid q_{i} \in \mathfrak{F}\right\}$ is infinite, or in other words, the run $r$ visits the set of final states $\mathfrak{F}$ infinitely often. Often in the literature, in order to emphasize that the infinite semantics is taken into consideration the automata are referred to as Büchi automata [PP04]. In our work we consider (Büchi) automata without the initial state specified and define the $(\omega$-)language in an automaton for any given state (see Section 3 for details).

2.1.1. Kleene theorems. Finite and infinite semantics of non-deterministic automata can be characterized in terms of two Kleene theorems (see e.g. [HMRU00, PP04]). The first statement is the following. A language $L \subseteq \Sigma^{*}$ is a language of finite words of an automaton $\mathcal{Q}$ (a.k.a. regular language) if and only if it is a rational language, i.e. it can be obtained from languages of the form $\varnothing$ and $\{a\}$ for any $a \in \Sigma$ by a sequence of applications of finite union, concatenation and Kleene star operation with the latter two given respectively by:

$$
\begin{aligned}
& R_{1} \cdot R_{2} \triangleq\left\{w_{1} w_{2} \mid w_{1} \in R_{1}, w_{2} \in R_{2}\right\}, \\
& R^{*} \triangleq\left\{w_{1} \ldots w_{n} \mid w_{i} \in R \text { and } n=0,1, \ldots\right\},
\end{aligned}
$$

for $R_{1}, R_{2}, R \subseteq \Sigma^{*}$.

The second Kleene theorem focuses on $\omega$-languages. A language $L_{\omega} \subseteq \Sigma^{\omega}$ is an $\omega$ language (a.k.a. $\omega$-regular language) of an automaton $\mathcal{Q}$ if and only if it is $\omega$-rational, i.e. it 
can be written as a finite union

$$
L_{\omega}=L_{1} \cdot R_{1}^{\omega} \cup \ldots \cup L_{n} \cdot R_{n}^{\omega},
$$

where $L_{i}, R_{i}$ are regular languages, the language $R_{i}^{\omega} \subseteq \Sigma^{\omega}$ is given by ${ }^{1}$ :

$$
R_{i}^{\omega} \triangleq\left\{\begin{array}{cc}
\left\{w_{1} w_{2} w_{3} \ldots \mid w_{i} \in R_{i}\right\} & \varepsilon \notin R_{i} \\
\Sigma^{\omega} & \text { otherwise }
\end{array}\right.
$$

and $L \cdot R_{\omega} \triangleq\left\{w v \mid w \in L, v \in R_{\omega}\right\}$ for $L \subseteq \Sigma^{*}$ and $R_{\omega} \subseteq \Sigma^{\omega}$.

2.2. Tree automata. There are several other variants of input for non-deterministic Büchi automata known in the literature [PP04, GTW02]. Here, we focus on non-deterministic (Büchi) tree automaton, i.e. a tuple $(Q, \Sigma, \delta, \mathfrak{F})$, where $\delta: Q \times \Sigma \rightarrow \mathcal{P}(Q \times Q)$ and the rest is as in the case of standard non-deterministic automata. The infinite semantics of this machine is given by a set of infinite binary trees with labels in $\Sigma$ for which there is a run whose every branch visits $\mathfrak{F}$ infinitely often [PP04, GTW02]. We recall these notions here below (with minor modifications to suit our language) and refer the reader to e.g. [PP04] for more details.

2.2.1. Trees. Formally, a binary tree or simply tree with nodes in $A$ is a function $t: P \rightarrow A$, where $P$ is a non-empty prefix closed subset of $\{l, r\}^{*}$. The set $P \subseteq\{l, r\}^{*}$ is called the domain of $t$ and is denoted by $\operatorname{dom}(t) \triangleq P$. Elements of $P$ are called nodes. For a node $w \in P$ any node of the form $w x$ for $x \in\{l, r\}$ is called a child of $w$. A tree is called complete if all nodes have either two children or no children. The height of a tree $t$ is $\max \{|w| \mid w \in \operatorname{dom}(t)\}$. A tree $t$ is finite if it is of a finite height, it is infinite if $\operatorname{dom}(t)=\{l, r\}^{*}$. The frontier of a tree $t$ is $\operatorname{fr}(t) \triangleq\{w \in \operatorname{dom}(t) \mid\{w l, w r\} \cap \operatorname{dom}(t)=\varnothing\}$. Elements of $\operatorname{fr}(t)$ are called leaves. Nodes from $\operatorname{dom}(t) \backslash \operatorname{fr}(t)$ are called inner nodes. The outer frontier of $t$ is defined by $\mathrm{fr}^{+}(t) \triangleq\{w l, w r \mid w \in \operatorname{dom}(t)\} \backslash \operatorname{dom}(t)$. I.e. it consists of all the words $w i \notin \operatorname{dom}(t)$ such that $w \in \operatorname{dom}(t)$ and $i \in\{l, r\}$. Finally, set $\operatorname{dom}^{+}(t) \triangleq \operatorname{dom}(t) \cup \mathrm{fr}^{+}(t)$.

Let $T_{\Sigma} X$ denote the set of all complete trees $t: P \rightarrow \Sigma+X$ with inner nodes taking values in $\Sigma$ and which have a finite number of leaves, all from the set $X$. Note that trees from $T_{\Sigma} X$ of height 0 can be thought of as elements of $X$. Hence, we may write $X \subseteq T_{\Sigma} X$. Moreover, trees of height 1 can be viewed as elements from $\Sigma \times X \times X$. Thus, $\Sigma \times X \times X \subseteq T_{\Sigma} X$. Additionally, any $f: X \rightarrow Y$ induces a map $T_{\Sigma} f: T_{\Sigma} X \rightarrow T_{\Sigma} Y$ which assigns to $t \in T_{\Sigma} X$ the tree obtained from $t$ by replacing any occurrence of a leaf $x \in X$ with $f(x) \in Y$. This turns $T_{\Sigma}(-)$ into a Set-endofunctor. For two functions $f: X \rightarrow T_{\Sigma} Y$ and $g: Y \rightarrow T_{\Sigma} Z$ we may naturally

$$
\begin{aligned}
& \Sigma=\{+,-\} \\
& f:\{x\} \rightarrow T_{\Sigma}\left\{y_{1}, y_{2}\right\} ; x \mapsto t \\
& g^{\prime}:\left\{y_{1}, y_{2}\right\} \rightarrow T_{\Sigma} Z ; y_{1} \mapsto t_{1}, y_{2} \mapsto t_{2} \\
& g^{\prime \prime}:\left\{y_{1}, y_{2}\right\} \rightarrow T_{\Sigma} Z ; y_{1} \mapsto t_{2}, y_{2} \mapsto t_{1} \\
& { }_{y}^{t}=\overbrace{y_{2}}^{\oplus} y_{1} \\
& g^{\prime} \cdot f(x)=\oplus_{t_{1}} g^{g^{\prime \prime} \cdot f(x)=\oplus_{t_{2}}}
\end{aligned}
$$

define $g \cdot f: X \rightarrow T_{\Sigma} Z$ for which $(g \cdot f)(x)$ is a tree obtained from $f(x)$ with every occurence of a variable $y \in Y$ replaced with the tree $g(y) \in T_{\Sigma} Z$. It is a simple exercise to prove that . is associative. Moreover, if we denote the function $X \rightarrow T_{\Sigma} X ; x \mapsto x$ by id then id $f=f$. id.

\footnotetext{
${ }^{1}$ Our definition of $R_{i}^{\omega}$ and the one presented in e.g. [PP04] differ slightly on $R_{i}$ with $\varepsilon \in R_{i}$. Indeed, in loc. cit., $R_{i}^{\omega}=\left\{w_{1} w_{2} \ldots \mid w_{i} \in R_{i}\right.$ and $\left.w_{i} \neq \varepsilon\right\}$. This small difference does not change the formulation of the Kleene theorem. We choose our definition of $(-)^{\omega}$ because it can be viewed as the greatest fixpoint of a certain assignment. See the following sections for details.
} 
This follows from the fact that $T_{\Sigma}$ is a monad and $g \cdot f$ is, in fact, the Kleisli composition for $T_{\Sigma}$ (see Example 4.9 for details).

Finally, $T_{\Sigma}^{*} X \subseteq T_{\Sigma} X$ and $T_{\Sigma}^{\omega} X \subseteq T_{\Sigma} X$ are sets of finite and infinite trees from $T_{\Sigma} X$ respectively. Note that trees in $T_{\Sigma}^{\omega} X$ have no leaves, hence $T_{\Sigma}^{\omega} X=T_{\Sigma}^{\omega} \varnothing$ for any set $X$.

2.2.2. Büchi tree automata and their languages. Let $\mathcal{Q}=(Q, \Sigma, \delta, \mathfrak{F})$ be a tree automaton. A run of the automaton $\mathcal{Q}$ on a finite tree $t \in T_{\Sigma} 1$ starting at the state $s \in Q$ is a map $\mathfrak{r}: \operatorname{dom}^{+}(t) \rightarrow Q$ such that $\mathfrak{r}(\varepsilon)=s$ and for any $x \in \operatorname{dom}(t) \backslash \operatorname{fr}(t)$ we have

$$
(\mathfrak{r}(x l), \mathfrak{r}(x r)) \in \delta(\mathfrak{r}(x), t(x)) .
$$

We say that the run $\mathfrak{r}$ is successful if $\mathfrak{r}(w) \in \mathfrak{F}$ for any $w \in \mathrm{fr}^{+}(t)$ for the tree $t$. The set of finite trees recognized by a state $s$ in $\mathcal{Q}$ is defined as the set of finite trees $t \in T_{\Sigma}^{*} 1$ for which there is a run in $\mathcal{Q}$ starting at $s$ which accepts the tree $t$.

Finally, let $t \in T_{\Sigma}^{\omega} \varnothing$ be an infinite tree with nodes in $\Sigma$. An infinite run for $t$ starting at $s \in Q$ is a map $\mathfrak{r}:\{l, r\}^{*} \rightarrow Q$ such that $\mathfrak{r}(\varepsilon)=s$ and:

$$
(\mathfrak{r}(x l), \mathfrak{r}(x r)) \in \delta(\mathfrak{r}(x), t(x)) \text { for all } x \in\{l, r\}^{*} .
$$

The tree $t$ is said to be recognized by the state $s$ in $\mathcal{Q}$ if there is a run $\mathfrak{r}$ for $t$ which start at $s$ and for each path in $t$ some final state occurs infinitely often [PP04].

2.2.3. Rational tree languages. Rational tree languages are analogues of rational languages for non-deterministic automata. Akin to the standard case, they are defined as sets of trees obtained from trees of height $\leq 1$ by a sequence of applications of: finite union, composition and Kleene star closure. However, the non-sequential nature of trees requires us to consider composition of rational trees with more than one variable.

Formally, for any subset $T \subseteq X \rightarrow T_{\Sigma} X$ we define $T^{*}$ by $T^{*} \triangleq \bigcup_{n} T^{n}$, where $T^{0}=\{$ id $\}$ and $T^{n}=T^{n-1} \cup\left\{t^{\prime} \cdot t \mid t^{\prime} \in T^{n-1}\right.$ and $\left.t \in T\right\}$. For any natural number $n \in\{0,1, \ldots\}$ we slightly abuse the notation and put $n \triangleq\{1, \ldots, n\}$ and define $\mathfrak{R a t}(1, n)$ to be the smallest family of subsets of $T_{\Sigma} n$ which satisfies:

- $\varnothing \in \mathfrak{R a t}(1, n)$,

- $\{t\} \in \mathfrak{R a t}(1, n)$, where $t$ is of height less than or equal to 1 ,

- if $T \in \mathfrak{R a t}(1, n)$ and $T_{1}, \ldots, T_{m} \in \mathfrak{R a t}(1, m)$ then:

$$
\left\{\left[t_{1}, \ldots, t_{n}\right] \cdot t \mid t \in T, t_{i} \in T_{i}\right\} \in \mathfrak{R a t}(1, m),
$$

- if $T \in \mathfrak{R a t}(1, n)$ then for any $i \in n$ :

$$
\left\{f(i) \mid f \in\left\{\left[t_{1}, \ldots, t_{n}\right]: n \rightarrow T_{\Sigma} n \mid t_{i} \in T\right\}^{*}\right\} \in \mathfrak{R a t}(1, n) .
$$

It is easy to check that if we extend the definition of $\mathfrak{R a t}$ and put

$$
\mathfrak{R a t}(m, n) \triangleq m \rightarrow \mathfrak{R a t}(1, n)
$$

then the last item in the above list implies that for any $T \in \mathfrak{R a t}(n, n)$ we have $T^{*} \in \mathfrak{R a t}(n, n)$.

Now for $T \subseteq n \rightarrow T_{\Sigma}^{*} n$ we define $T^{\omega}$ as the subset of $n \rightarrow T_{\Sigma}^{\omega} \varnothing$ consisting of common extensions of functions in $T^{k}$ for any $k$. Finally, the $\omega$-rational subset of trees is defined by [PP04]:

$$
\omega \mathfrak{R a t} \triangleq\left\{T^{\omega} \cdot T^{\prime} \mid T \in \mathfrak{R a t}(n, n) \text { and } T^{\prime} \in \mathfrak{R a t}(1, n)\right\},
$$

where $T \cdot T^{\prime} \triangleq\left\{t \cdot t^{\prime} \mid t \in T, t^{\prime} \in T^{\prime}\right\}$. 
2.2.4. Kleene theorems. Let $\mathfrak{R e g}$ be the set of subsets of trees from $T_{\Sigma}^{*} 1$ for which there is an automaton accepting the given set of trees. Similarly, we define the set $\omega \mathfrak{R e g}$ of infinite trees accepted by the tree automata. In this case the Kleene theorems for regular and $\omega$-regular input are respectively given by [PP04]:

$$
\mathfrak{R e g}=\mathfrak{R a t}(1,1) \text { and } \omega \mathfrak{R e g}=\omega \mathfrak{R a t} .
$$

In Section 3 we will show that Kleene theorems for non-deterministic automata and tree automata are instances of a generic pair of theorems formulated on a categorical level.

2.3. Algebras and coalgebras. Let $F: \mathrm{C} \rightarrow \mathrm{C}$ be a functor. An $F$-coalgebra ( $F$-algebra) is a morphism $\alpha: A \rightarrow F A$ (resp. $a: F A \rightarrow A$ ). The object $A$ is called a carrier of the underlying $F$-(co)algebra. Given two coalgebras $\alpha: A \rightarrow F A$ and $\beta: B \rightarrow F B$ a morphism $h: A \rightarrow B$ is homomorphism from $\alpha$ to $\beta$ provided that $\beta \circ h=F(h) \circ \alpha$. For two algebras $a: F A \rightarrow A$ and $b: F B \rightarrow B$ a morphism $h: A \rightarrow B$ is called homomorphism from $a$ to $b$ if $b \circ F(h)=h \circ a$. The category of all $F$-coalgebras ( $F$-algebras) and homomorphisms between them is denoted by $\operatorname{CoAlg}(F)$ (resp. $\operatorname{Alg}(F)$ ). We say that a coalgebra $\zeta: Z \rightarrow F Z$ is final or terminal if for any $F$-coalgebra $\alpha: A \rightarrow F A$ there is a unique homomorphism $[[\alpha]]: A \rightarrow Z$ from $\alpha$ to $\zeta$.

Example 2.1. Let $\Sigma$ be a set of labels. Labelled transition systems (see e.g. [San11]) can be viewed as coalgebras of the type $\mathcal{P}(\Sigma \times \mathcal{I} d):$ Set $\rightarrow$ Set [Rut00]. Here, $\mathcal{P}:$ Set $\rightarrow$ Set is the powerset functor which maps any $X$ to the set $\mathcal{P} X=\{A \mid A \subseteq X\}$ and any $f: X \rightarrow Y$ to $\mathcal{P} f: \mathcal{P} X \rightarrow \mathcal{P} Y ; A \mapsto f(A)$.

Non-deterministic automata as defined in Subsection 2.1 are modelled as coalgebras of the type $\mathcal{P}(\Sigma \times \mathcal{I} d+1)$, where $1=\{\checkmark\}$ (e.g. [HJS07]). Indeed, any non-deterministic automaton $(Q, \Sigma, \delta, \mathfrak{F})$ is modelled by $\alpha: Q \rightarrow \mathcal{P}(\Sigma \times Q+1)$ where:

$$
\alpha(q)=\left\{\left(a, q^{\prime}\right) \mid q^{\prime} \in \delta(a, q)\right\} \cup \chi(q),
$$

where $\chi(q)=\left\{\begin{array}{cc}\{\checkmark\} & q \in \mathfrak{F}, \\ \varnothing & \text { otherwise. }\end{array}\right.$ In a similar manner, we can model tree automata coalgebraically, i.e. as coalgebras of the type $Q \rightarrow \mathcal{P}(\Sigma \times Q \times Q+1)$.

Example 2.2. Fully probabilistic processes [BH97] sometimes referred to as fully probabilistic systems [Sok11] are modelled as $\mathcal{D}(\Sigma \times \mathcal{I} d)$-coalgebras [Sok11]. Here, $\mathcal{D}$ denotes the subdistribution functor assigning to any set $X$ the set $\left\{\mu: X \rightarrow[0,1] \mid \sum_{x} \mu(x) \leq 1\right\}$ of subdistributions with countable support and to any map $f: X \rightarrow Y$ the map $\mathcal{D} f: \mathcal{D} X \rightarrow$ $\mathcal{D} Y ; \mu \mapsto \mathcal{D} f(\mu)$ with

$$
\mathcal{D} f(\mu)(y)=\sum\{\mu(x) \mid x \in X \text { such that } f(x)=y\} .
$$

2.4. Monads. For a general introduction to the theory of monads the reader is referred to e.g. [BW02, ML78]. A monad on $\mathrm{C}$ is a triple $(T, \mu, \eta)$, where $T: \mathrm{C} \rightarrow \mathrm{C}$ is an endofunctor and $\mu: T^{2} \Longrightarrow T, \eta: \mathcal{I} d \Longrightarrow T$ are two natural transformations for which the following 
diagrams commute:
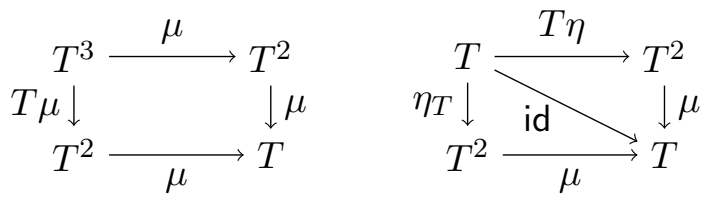

The transformation $\mu$ is called multiplication and the transformation $\eta$ is called unit.

For any monad $(T: \mathrm{C} \rightarrow \mathrm{C}, \mu, \eta)$ we define the Kleisli category $\mathcal{K l}(T)$ for $T$ has whose class of objects is the class of objects of $\mathrm{C}$ and for two objects $X, Y$ in $\mathcal{K} l(T)$ we put $\mathcal{K} l(T)(X, Y)=\mathrm{C}(X, T Y)$ with the composition $\cdot$ in $\mathcal{K} l(T)$ defined between two morphisms $f: X \rightarrow T Y$ and $g: Y \rightarrow T Z$ by $g \cdot f:=\mu_{Z} \circ T(g) \circ f$. Since most of the time we work with two categories at once, namely $\mathrm{C}$ and $\mathcal{K} l(T)$, morphisms in $\mathrm{C}$ will be denoted using standard arrows $\rightarrow$, whereas for morphisms in $\mathcal{K} l(T)$ we will use the symbol $\rightarrow \rightarrow$. Hence, $f: X \multimap \rightarrow=X \rightarrow T Y$ and the composition is given by

$$
X \stackrel{f}{\rightarrow} Y \stackrel{g}{\rightarrow} Y Z=X \stackrel{f}{\rightarrow} T Y \stackrel{T g}{\rightarrow} T T Z \stackrel{\mu_{Z}}{\rightarrow} T Z .
$$

We define a functor $\sharp: C \rightarrow \mathcal{K} l(T)$ which sends each object $X \in \mathrm{C}$ to itself and each morphism $f: X \rightarrow Y$ in $C$ to the morphism $f^{\sharp}: X \rightarrow T Y ; f^{\sharp} \triangleq \eta_{Y} \circ f$. Maps in $\mathcal{K} l(T)$ of the form $f^{\sharp}$ for some $f: X \rightarrow Y \in \mathrm{C}$ are referred to as base morphisms.

Every monad $(T, \mu, \eta)$ on a category $\mathrm{C}$ arises from the composition of a left and a right adjoint: $\mathrm{C} \leftrightarrows \mathcal{K} l(T)$, where the left adjoint is ${ }^{\sharp}: \mathrm{C} \rightarrow \mathcal{K l}(T)$ and the right adjoint $U_{T}: \mathcal{K l}(T) \rightarrow \mathrm{C}$ is defined as follows: for any object $X \in \mathcal{K} l(T)$ (i.e. $X \in \mathrm{C}$ ) the object $U_{T} X$ is given by $U_{T} X:=T X$ and for any morphism $f: X \rightarrow T Y$ in $\mathcal{K} l(T)$ the morphism $U_{T} f: T X \rightarrow T Y$ is given by $U_{T} f=\mu_{Y} \circ T f$.

We say that a functor $F: C \rightarrow C$ lifts to a functor $\bar{F}: \mathcal{K} l(T) \rightarrow \mathcal{K} l(T)$ provided that the following diagram commutes:

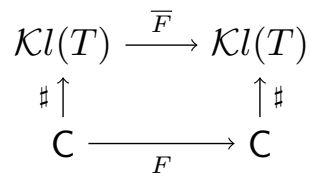

There is a one-to-one correspondence between liftings $\bar{F}$ and distributive laws $\lambda: F T \Longrightarrow T F$ between the functor $F$ and the monad $T^{2}$. Indeed, any lifting $\bar{F}: \mathcal{K l}(T) \rightarrow \mathcal{K l}(T)$ induces the transformation $\lambda$ whose $X$-component $\lambda_{X}: F T X \rightarrow T F X$ is $\lambda_{X}=\bar{F}\left(\mathrm{id}_{T X}: T X \rightarrow T X\right)$ and any distributive law $\lambda: F T \Longrightarrow T F$ gives rise to a lifting $\bar{F}: \mathcal{K} l(T) \rightarrow \mathcal{K} l(T)$ given by:

$$
\bar{F} X=F X \text { and } \bar{F}(X \stackrel{f}{\rightarrow} T Y)=F X \stackrel{F f}{\rightarrow} F T Y \stackrel{\lambda_{Y}}{\rightarrow} T F Y .
$$

A monad $(T, \mu, \eta)$ on a category $\mathrm{C}$ with finite products is called strong if there is a natural transformation $t_{X, Y}: X \times T Y \rightarrow T(X \times Y)$ called tensorial strength satisfying the strength laws listed in e.g. [Koc72]. Existence of strength guarantees that for any object $\Sigma$ the functor $\Sigma \times \mathcal{I} d: C \rightarrow C$ admits a lifting $\bar{\Sigma}: \mathcal{K} l(T) \rightarrow \mathcal{K} l(T)$ defined as follows. For any $X \in \mathcal{K} l(T)$ we put $\bar{\Sigma} X:=\Sigma \times X$, and for any $f: X \multimap \rightarrow Y=X \rightarrow T Y$ we define $\bar{\Sigma} f \triangleq t_{\Sigma, Y} \circ\left(i d_{\Sigma} \times f\right)$. Existence of the transformation $t$ is not a strong requirement. For instance all monads on Set are strong.

\footnotetext{
${ }^{2}$ A distributive law between a functor $F$ and a monad $T$ is a natural transformation $F T \Longrightarrow T F$ which additionally satisfies extra conditions listed in e.g. [JSS12, Mul93].
} 
Example 2.3. The powerset endofunctor $\mathcal{P}:$ Set $\rightarrow$ Set, used in the definition of labelled transition systems, non-deterministic automata and tree automata, carries a monadic structure $(\mathcal{P}, \bigcup,\{-\})$ for which the multiplication and the unit are given by:

$$
\bigcup: \mathcal{P} \mathcal{P} X \rightarrow \mathcal{P} X ; S \mapsto \bigcup S, \quad\{-\}: X \rightarrow \mathcal{P} X ; x \mapsto\{x\}
$$

The Kleisli category $\mathcal{K} l(\mathcal{P})$ consists of sets as objects and morphisms given by the maps $f: X \multimap \neg Y=X \rightarrow \mathcal{P} Y$ and $g: Y \multimap \rightarrow Z=Y \rightarrow \mathcal{P} Z$ with the composition $g \cdot f: X \multimap \neg Z=$ $X \rightarrow \mathcal{P} Z$ given by

$$
(g \cdot f)(x)=\bigcup_{y \in f(x)} g(y)
$$

The identity morphisms id : $X \multimap X=X \rightarrow \mathcal{P} X$ are given for any $x \in X$ by id $(x)=\{x\}$. The Kleisli category for $\mathcal{P}$ is isomorphic to Rel - the category of sets as objects, and relations as morphisms. The $X$-component of the distributive law $\lambda: \Sigma \times \mathcal{P} X \rightarrow \mathcal{P}(\Sigma \times X)$ induced by strength of $\mathcal{P}$ is:

$$
\lambda\left(a, X^{\prime}\right)=\left\{(a, x) \mid x \in X^{\prime}\right\} .
$$

Example 2.4. The subdistribution functor $\mathcal{D}:$ Set $\rightarrow$ Set from Example 2.2 carries a monadic structure $(\mathcal{D}, \mu, \eta)$, where $\mu_{X}: \mathcal{D D} X \rightarrow \mathcal{D} X$ is

$$
\mu(\psi)(x)=\sum_{\phi \in \mathcal{D} X} \psi(\phi) \cdot \phi(x)
$$

and $\eta_{X}: X \rightarrow \mathcal{D} X$ assigns to any $x$ the Dirac delta distribution $\delta_{x}: X \rightarrow[0,1]$.

Example 2.5. For any monoid $(M, \cdot, 1)$ the Set-functor $M \times \mathcal{I} d$ carries a monadic structure $(M \times \mathcal{I} d, m, e)$, where $m_{X}: M \times M \times X \rightarrow M \times X ;(m, n, x) \mapsto(m \cdot n, x)$ and $e_{X}: X \rightarrow$ $M \times X ; x \mapsto(1, x)$.

From the perspective of this paper, the most imporant instance of the family of monads from Example 2.5 is the monad $\left(\Sigma^{*} \times \mathcal{I} d, m, e\right)$, where $\left(\Sigma^{*}, \cdot, \varepsilon\right)$ is the free monoid over $\Sigma$. The reason is that $\Sigma^{*} \times \mathcal{I} d$ is the free monad over the functor $\Sigma \times \mathcal{I} d$ and hence, since $\Sigma \times \mathcal{I} d$ lifts to the Kleisli category for any Set-based monad $T$ (since all Set-based monads are strong), then so does $\Sigma^{*} \times \mathcal{I} d$ whose lifting is the free monad over the lifting of $\Sigma \times \mathcal{I} d$ [Bre15]. In practice, this yields a monadic structure on $T\left(\Sigma^{*} \times \mathcal{I} d\right)$ for any monad $T$ on the category of sets [Bre15].

Example 2.6. If $T=\mathcal{P}$ then the Kleisli category for $\mathcal{P}\left(\Sigma^{*} \times \mathcal{I} d\right)$ has the composition given as follows [Bre15]. For two morphisms $f: X \multimap Y=X \rightarrow \mathcal{P}\left(\Sigma^{*} \times Y\right)$ and $g: Y \multimap Z=$ $Y \rightarrow \mathcal{P}\left(\Sigma^{*} \times Z\right)$ we have

$$
g \cdot f(x)=\left\{\left(\sigma_{1} \sigma_{2}, z\right) \mid x{\stackrel{\sigma_{1}}{\rightarrow}}_{f} y{\stackrel{\sigma_{2}}{\rightarrow}}_{g} z \text { for some } y \in Y\right\} .
$$

The identity morphisms in this category are id : $X \multimap \rightarrow X=X \rightarrow \mathcal{P}\left(\Sigma^{*} \times X\right)$ given by $\operatorname{id}(x)=\{(\varepsilon, x)\}$.

In a similar manner, using the remark above, we show that $\mathcal{D}\left(\Sigma^{*} \times \mathcal{I} d\right)$ carries a monadic structure. 
2.5. Coalgebras with internal moves. Coalgebras with internal moves were first introduced in the context of coalgebraic trace semantics as coalgebras of the type $T F_{\varepsilon}$ for a monad $T$ and an endofunctor $F$ on $C$ with $F_{\varepsilon}$ defined by $F_{\varepsilon} \triangleq F+\mathcal{I} d$ [Has06, SW13]. If we take $F=\Sigma \times \mathcal{I} d$ then we have $T F_{\varepsilon}=T(\Sigma \times \mathcal{I} d+\mathcal{I} d) \cong T\left(\Sigma_{\varepsilon} \times \mathcal{I} d\right)$, where $\Sigma_{\varepsilon} \triangleq \Sigma+\{\varepsilon\}$. In [Bre15] we showed that given certain assumptions on $T$ and $F$ we may embed the functor $T F_{\varepsilon}$ into the monad $T F^{*}$, where $F^{*}$ is the free monad over $F$. In particular, if we apply this construction to $T=\mathcal{P}$ and $F=\Sigma \times \mathcal{I} d$ we obtain the monad $\mathcal{P}\left(\Sigma^{*} \times \mathcal{I} d\right)$ from Example 2.6. The construction of $T F^{*}$ is revisited in this paper in Section 4. The trick of modelling the invisible steps via a monadic structure allows us not to specify the internal moves explicitly. Instead of considering $T F_{\varepsilon}$-coalgebras we consider $T^{\prime}$-coalgebras for a monad $T^{\prime}$ on an arbitrary category.

The strategy of finding a suitable monad (for modelling the behaviour taken into consideration) will also be applied in this paper. Unfortunately, from the point of view of the infinite behaviour of coalgebras, considering systems of the type $T F^{*}$ is not sufficient (see Section 3 for a discussion). Hence, in Section 4 we show how to obtain a monad suitable for modelling infinite behaviour. Intuitively, the new monad extends $T F^{*}$ by adding an ingredient associated with the terminal $F$-coalgebra $\zeta: F^{\omega} \rightarrow F F^{\omega}$. The construction presented in Section 4 yields the monad $T F^{\infty}=T\left(F^{*} \oplus F^{\omega}\right)$ suitable to capture both: finite and infinite behaviour of systems. Below we give two examples of such monad.

Example 2.7. Although the monad $\mathcal{P}\left(\Sigma^{*} \times \mathcal{I} d\right)$ from Example 2.6 proves to be sufficient to model finite behaviours of non-deterministic automata (see [Bre15, BMP15]), it will not be suitable to model their infinite behaviour (see Section 3 for details). Hence, we extend $\mathcal{P}\left(\Sigma^{*} \times \mathcal{I} d\right)$ and consider the following. Let $\Sigma^{\omega}$ be the set of all infinite sequences of elements from $\Sigma$. As it will be shown in sections to come, the functor $\mathcal{P}\left(\Sigma^{*} \times \mathcal{I} d+\Sigma^{\omega}\right)$ carries a monadic structure whose Kleisli composition is as follows. For $f: X \rightarrow \mathcal{P}\left(\Sigma^{*} \times Y+\Sigma^{\omega}\right)$ and $g: Y \rightarrow \mathcal{P}\left(\Sigma^{*} \times Z+\Sigma^{\omega}\right)$ the map $g \cdot f: X \multimap \rightarrow=X \rightarrow \mathcal{P}\left(\Sigma^{*} \times Z+\Sigma^{\omega}\right)$ satisfies:

$$
\begin{aligned}
& x \stackrel{\sigma}{\rightarrow}_{g \cdot f} z \Longleftrightarrow \exists y \text { such that } x{\stackrel{\sigma_{f}}{\rightarrow}}_{f} \text { and } y{\stackrel{\sigma_{2}}{\rightarrow}}_{g} z, \text { where } \sigma=\sigma_{1} \sigma_{2} \in \Sigma^{*}, \\
& x \downarrow_{g \cdot f} v \Longleftrightarrow x \downarrow_{f} v \text { or } x \stackrel{\sigma}{\rightarrow}_{f} y \text { with } y \downarrow_{g} v^{\prime} \text { and } v=\sigma v^{\prime} \in \Sigma^{\omega} .
\end{aligned}
$$

In the above we write $x \stackrel{\sigma}{\rightarrow}_{f} y$ whenever $(\sigma, y) \in f(x)$ and $x \downarrow_{f} v$ if $v \in f(x)$ for $\sigma \in \Sigma^{*}$, $v \in \Sigma^{\omega}$. The identity morphisms in this category are the same as in the Kleisli category for the monad $\mathcal{P}\left(\Sigma^{*} \times \mathcal{I} d\right)$. The monadic structure of $\mathcal{P}\left(\Sigma^{*} \times \mathcal{I} d+\Sigma^{\omega}\right)$ arises as a consequence of a general construction of monads modelling (in)finite behaviour described in detail in Section 4 .

Example 2.8. If we move from non-deterministic automata towards tree automata we have to find a suitable monadic setting to talk about their (in)finite behaviour. It turns out that a good candidate for this monad can be built from the ingredients already presented in this paper. Indeed, if we take the powerset monad and the monad $T_{\Sigma}$ from Subsection 2.2.1, then their composition $\mathcal{P} T_{\Sigma}$ carries a monadic structure ${ }^{3}$. The formula for the composition in the Kleisli category for the monad $\mathcal{P} T_{\Sigma}$ is given for $f: X \rightarrow \mathcal{P} T_{\Sigma} Y$ and $g: Y \rightarrow \mathcal{P} T_{\Sigma} Z$ by $g \cdot f: X \multimap Z=X \rightarrow \mathcal{P} T_{\Sigma} Z$ with $g \cdot f(x)$ being a set of trees obtained from trees in $f(x) \subseteq T_{\Sigma} Y$ by replacing any occurence of the leaf $y \in Y$ with a tree from $g(y) \subseteq T_{\Sigma} Z$. As will be witnessed in Section 4, this monad and the monad $\mathcal{P}\left(\Sigma^{*} \times \mathcal{I} d+\Sigma^{\omega}\right)$ arise from the same categorical construction.

\footnotetext{
${ }^{3}$ The proof of this claim can be found in Section 4. See Example 4.9 for details.
} 
The list of examples of monads used in the paper will be extended in the upcoming sections.

2.6. Categorical order enrichment. Our main ingredients for defining (in)finite behaviours of automata will turn out to be two fixpoint operators: $(-)^{*}$ and $(-)^{\omega}$. In order to establish them on a categorical level we require the category under consideration to be suitably order enriched. A category is said to be order enriched, or simply ordered, if each hom-set is a poset with the order preserved by the composition. It is $V$-ordered if all hom-posets admit arbitrary finite (possibly empty) suprema. Note that, given such suprema exist, the composition in $\mathrm{C}$ does not have to distribute over them in general. We call a category left distributive (or $L D$ in $f-\otimes-g-=-f \vee g$ short) if it is $\vee$-ordered and $g \cdot\left(\bigvee_{i \in I} f_{i}\right)=\bigvee_{i \in I} g \cdot f_{i}$ for any finite set $I$. We define right distributivity analogously. In this paper we come across many left distributive categories that do not necessarily satisfy right distributivity. Still, however, all examples of Kleisli categories taken into consideration satisfy its weaker form. To be more precise, we say that the Kleisli category $\mathcal{K l}(T)$ for a monad $T$ on $\mathrm{C}$ is right distributive w.r.t. base morphisms provided that $\left(\bigvee_{i \in I} f_{i}\right) \cdot j^{\sharp}=\bigvee_{i \in I} f_{i} \cdot j^{\sharp}$ for any $f_{i}: Y \multimap \rightarrow Z=Y \rightarrow T Z \in \mathcal{K} l(T)(Y, Z)$, any $j: X \rightarrow Y \in \mathrm{C}(X, Y)$ and any finite set $I$. We say that an order enriched category is $\omega$ Cpo-enriched if any countable ascending chain of morphisms $f_{1} \leq f_{2} \leq \ldots$ with common domain and codomain admits a supremum which is preserved by the morphism composition. Finally, in an ordered category with finite coproducts we say that cotupling preserves order if $\left[f_{1}, f_{2}\right] \leq\left[g_{1}, g_{2}\right] \Longleftrightarrow f_{1} \leq g_{1}$ and $f_{2} \leq g_{2}$ for any $f_{i}, g_{i}$ with suitable domains and codomains.

Remark 2.9. Right distributivity w.r.t. the base morphisms and cotupling order preservation are properties we often get as a consequence of other general assumptions. Indeed, any Set-based monad $T$ whose order enrichment of the Kleisli category is given by $f \leq g \Longleftrightarrow$ $\forall x . f(x) \leq g(x)$, for $f, g: X \rightarrow T Y$ where $T Y$ is a poset for any $Y^{4}$, satisfies these conditions. Note that, in this case, the Kleisli composition over any suprema or infima that exist is right distributive w.r.t. morphisms of the form $j^{\sharp}=\eta_{Y} \circ j: X \multimap \rightarrow Y=X \rightarrow T Y$ for any set map $j: X \rightarrow Y$. A similar argument applies to cotupling order preservation.

Example 2.10. The next section of this paper focuses on three categories, namely: $\mathcal{K l}\left(\mathcal{P}\left(\Sigma^{*} \times\right.\right.$ $\mathcal{I} d)), \mathcal{K} l\left(\mathcal{P}\left(\Sigma^{*} \times \mathcal{I} d+\Sigma^{\omega}\right)\right)$ and $\mathcal{K} l\left(\mathcal{P} T_{\Sigma}\right)$. These categories are order-enriched with the hom-set ordering given by $f \leq g \Longleftrightarrow f(x) \subseteq g(x)$ for any $x$. The base morphisms of the first two examples are of the form

$$
X \rightarrow \mathcal{P}(\{\varepsilon\} \times Y) ; x \mapsto\{(\varepsilon, j(x))\}
$$

for a set map $j: X \rightarrow Y$. The base morphisms of the third example are given by $X \rightarrow$ $\mathcal{P} T_{\Sigma} Y ; x \mapsto\{j(x)\}$. We leave it as an exercise to the reader to verify that all these examples satisfy the following conditions: the order enrichment is pointwise induced; they are $\omega$ Cpoenriched; their hom-sets are complete lattices; they are left distributive ${ }^{5}$. These conditions play a central role in defining (in)finite behaviours on a coalgebraic level. We will elaborate more on them in Section 5.

\footnotetext{
${ }^{4}$ In this case, we say that the order enrichement of $\mathcal{K} l(T)$ is pointwise induced.

${ }^{5}$ We refer the reader to [Bre15] for a proof that $\mathcal{K} l\left(\mathcal{P}\left(\Sigma^{*} \times \mathcal{I} d\right)\right)$ satisfies these conditions.
} 
2.7. Lawvere theories. The primary interest of the theory of automata and formal languages focuses on automata over a finite state space. Hence, since we are interested in systems with internal moves (i.e. coalgebras $X \rightarrow T X$ for a monad $T$ ), without loss of generality we may focus our attention on coalgebras of the form $n \rightarrow T n$, where $n=\{1, \ldots, n\}$ with $n=0,1, \ldots$. These morphisms are endomorphisms in a full subcategory of the Kleisli category for $T$, we will later refer to as (Lawvere) theory. Restricting the scope to this category instead of considering the whole Kleisli category for a given monad plays an important role in Kleene theorems characterizing regular and $\omega$-regular behaviour (see e.g. [HMRU00, PP04]).

Formally, a Lawvere theory, or simply theory, is a category whose objects are natural numbers $n \geq 0$ such that each $n$ is an $n$-fold coproduct of 1 . The definition used here is dual to the classical notion [Law63] and can be found in e.g. [ÉK11, ÉK13, EH09]. The reason why we use our version of the definition is the following: we want the connection between Lawvere theories and Kleisli categories for Set-based monads to be as direct as possible. Indeed, in our case, any monad $T$ on Set induces a theory $\mathbb{T}$ associated with it by restricting the Kleisli category $\mathcal{K} l(T)$ to objects $n$ for any $n \geq 0$. Conversely, for any theory $\mathbb{T}$ there is a Set based monad the theory is associated with (see e.g. [HP07] for details). This remark also motivates us to use the notation introduced before and denote morphisms from a theory by $\rightarrow \rightarrow$.

For any element $i \in n$ let $i_{n}: 1 \multimap n$ denote the $i$-th coproduct injection and $\left[f_{1}, \ldots, f_{k}\right]: n_{1}+\cdots+n_{k} \rightarrow n$ the cotuple of $\left\{f_{l}: n_{l} \multimap \rightarrow n\right\}_{l}$ depicted in the diagram on the right. The coprojection morphism $n_{i} \rightarrow n_{1}+\cdots+n_{k}$ into the $i$-th component of the coproduct will be denoted by $\operatorname{in}_{n_{1}+\cdots+n_{k}}^{n_{i}}$. Any morphism $k \rightarrow n$ of the form $\left[\left(i^{1}\right)_{n}, \ldots,\left(i^{k}\right)_{n}\right]: k \rightarrow \circ$ for $i^{j} \in n$ is called

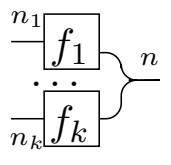
base morphism or base map. If $\mathbb{T}$ is associated with a monad $T$ then the base morphisms in $\mathbb{T}$ are exactly given by $f^{\sharp}: m \rightarrow n=m \rightarrow T n$ for Set-maps $f: m \rightarrow n$. Finally, let ! $: n \rightarrow 1$ be defined by $! \triangleq\left[1_{1}, 1_{1}, \ldots, 1_{1}\right]$. We say that a theory $\mathbb{T}^{\prime}$ is a subtheory of $\mathbb{T}$ if there is a faithful functor $\mathbb{T}^{\prime} \rightarrow \mathbb{T}$ which maps any object $n$ onto itself.

Example 2.11. By LTS, LTS ${ }^{\omega}$ and TTS ${ }^{\omega}$ we denote the theories associated with the monads $\mathcal{P}\left(\Sigma^{*} \times \mathcal{I} d\right), \mathcal{P}\left(\Sigma^{*} \times \mathcal{I} d+\Sigma^{\omega}\right)$ and $\mathcal{P} T_{\Sigma}$ respectively.

\section{Non-DEterministic AUtomata, COALGEBraicAlly}

The purpose of this section is to give motivations for the abstract theory presented in the remainder of the paper. In the first part of this section we focus on finite non-deterministic (Büchi) automata and their (in)finite behaviour from the perspective of the categories $\mathcal{K} l\left(\mathcal{P}\left(\Sigma^{*} \times \mathcal{I} d\right)\right)$ and $\mathcal{K} l\left(\mathcal{P}\left(\Sigma^{*} \times \mathcal{I} d+\Sigma^{\omega}\right)\right)$. Afterwards, we deal with tree automata and their behaviour. Finally, we give a categorical perspective on Kleene theorems for automata taken into consideration.

3.1. Non-deterministic automata. Without any loss of generality we may only consider automata over the state space $n=\{1, \ldots, n\}$ for some natural number $n$. As mentioned in Example 2.1 any non-deterministic automaton $(n, \Sigma, \delta, \mathfrak{F})$ may be modelled as a $\mathcal{P}(\Sigma \times \mathcal{I} d+$ 1 )-coalgebra $n \rightarrow \mathcal{P}(\Sigma \times n+1)$ [Rut00]. However, as it has been already noted in [USH16], from the point of view of infinite behaviour with $\mathrm{BAC}$ it is more useful to extract the information about the final states of the automaton and not to encode it into the transition 
map as above. Instead, given an automaton $(n, \Sigma, \delta, \mathfrak{F})$ we encode it as a pair $(\alpha, \mathfrak{F})$ where $\alpha: n \rightarrow \mathcal{P}(\Sigma \times n)$ is defined by $\alpha(i)=\{(a, j) \mid j \in \delta(a, i)\}$ and consider the map:

$$
\mathfrak{f}_{\mathfrak{F}}: n \rightarrow \mathcal{P}(\{\varepsilon\} \times n) ; i \mapsto\left\{\begin{array}{cc}
\{(\varepsilon, i)\} & \text { if } i \in \mathfrak{F} \\
\varnothing & \text { otherwise }
\end{array}\right.
$$

Note that by extending the codomain of $\alpha$ and $\mathfrak{f}_{\mathfrak{F}}$ both maps can be viewed as endomorphisms in LTS and LTS ${ }^{\omega}$. The purpose of $\mathfrak{f}_{\mathfrak{F}}$ is to encode the set of accepting states with an endomorphism in the same Kleisli category in which the transition $\alpha$ is an endomorphism. Now, we have all the necessary ingredients to revisit finite and infinite behaviour (with BAC) of non-deterministic automata from the perspective of the theories LTS and LTS ${ }^{\omega}$.

3.1.1. Finite behaviour. Consider $\alpha^{*}: n \rightarrow n$ to be an endomorphism in LTS (or LTS ${ }^{\omega}$ ) given by $\alpha^{*}=\mu x$. (id $\left.\vee x \cdot \alpha\right)=\bigvee_{n \in \omega} \alpha^{n}$, where the order is as in Example 2.11. We have [Bre15]:

$$
\alpha^{*}(i)=\{(\sigma, j) \mid i \stackrel{\sigma}{\Longrightarrow} j\},
$$

where $\stackrel{\sigma}{\Longrightarrow} \triangleq(\stackrel{\varepsilon}{\rightarrow})^{*} \circ \stackrel{a_{1}}{\rightarrow} \circ(\stackrel{\varepsilon}{\rightarrow})^{*} \circ \ldots(\stackrel{\varepsilon}{\rightarrow})^{*} \circ \stackrel{a_{n}}{\rightarrow}(\stackrel{\varepsilon}{\rightarrow})^{*}$ for $\sigma=a_{1} \ldots a_{n}, a_{i} \in \Sigma$ and $\stackrel{\varepsilon}{\longrightarrow} \triangleq(\stackrel{\varepsilon}{\rightarrow})^{*}$. Let us observe that the theory morphism ! : $n \rightarrow 1$ is explicitly given in the case of theories LTS and $\operatorname{LTS}^{\omega}$ by $!(i)=\{(\varepsilon, 1)\}$ for any $i \in n$. Finally, consider the morphism $! \cdot \mathfrak{f}_{\mathfrak{F}} \cdot \alpha^{*}: n \rightarrow 1$ in LTS (or LTS ${ }^{\omega}$ ) which is:

$$
! \cdot \mathfrak{f}_{\mathfrak{F}} \cdot \alpha^{*}(i)=\left\{(\sigma, 1) \mid \sigma \in \Sigma^{*} \text { such that } i \stackrel{\sigma}{\Longrightarrow} j \text { and } j \in \mathfrak{F}\right\} .
$$

Since $\mathcal{P}\left(\Sigma^{*} \times 1\right) \cong \mathcal{P}\left(\Sigma^{*}\right)$, the set $! \cdot \mathfrak{f}_{\mathfrak{F}} \cdot \alpha^{*}(i)$ represents the set of all finite words accepted by the state $i$ in the automaton $(n, \Sigma, \delta, \mathfrak{F})$.

3.1.2. Infinite behaviour. Note that the hom-posets of theories LTS and LTS ${ }^{\omega}$ are complete lattices and, hence (by the Tarski-Knaster theorem), come equipped with an operator which assigns to any endomorphism $\beta: n \rightarrow n$ the morphism $\beta^{\omega}: n \rightarrow 0$ defined as the greatest fixpoint of the assignment $x \mapsto x \cdot \beta$. Now, if $\alpha$ is given as in the previous subsections and considered as an endomorphism in the theory LTS then the map $\alpha^{\omega}: n \multimap \rightarrow 0=n \rightarrow \mathcal{P}\left(\Sigma^{*} \times \varnothing\right)$ in LTS satifies $\alpha^{\omega}(i)=\varnothing$. However, if we consider $\alpha$ to be an endomorphism in LTS $^{\omega}$ and compute $\alpha^{\omega}: n \rightarrow 0=n \rightarrow \mathcal{P}\left(\Sigma^{*} \times \varnothing+\Sigma^{\omega}\right)=n \rightarrow \mathcal{P}\left(\Sigma^{\omega}\right)$ in LTS ${ }^{\omega}$ the result will be different. Indeed, we have the following.

Proposition 3.1. Let $\beta: n \rightarrow \mathcal{P}\left(\Sigma^{*} \times n\right)$ and, since $\mathcal{P}\left(\Sigma^{*} \times n\right) \subseteq \mathcal{P}\left(\Sigma^{*} \times n+\Sigma^{\omega}\right)$, it can be considered as an endomorphism in $\mathrm{LTS}^{\omega}$. In this case, the explicit formula for the greatest fixpoint $\beta^{\omega}: n \rightarrow 0=n \rightarrow \mathcal{P}\left(\Sigma^{\omega}\right)$ of the assignment $x \mapsto x \cdot \beta$ calculated in $\mathrm{LTS}^{\omega}$ is given by:

$$
\beta^{\omega}(i)=\bigcup\left\{\left|\sigma_{1}, \sigma_{2}, \ldots\right| \subseteq \Sigma^{\omega} \mid i \stackrel{\sigma}{\rightarrow}_{\beta} i_{1}{\stackrel{\sigma_{2}}{\rightarrow}}_{\beta} i_{2} \ldots \text { for some } i_{k} \in n \text { and } \sigma_{k} \in \Sigma^{*}\right\},
$$

where $|-|:\left(\Sigma^{*}\right)^{\omega} \rightarrow \mathcal{P}\left(\Sigma^{\omega}\right)$ assigns to any sequence $\sigma_{1}, \sigma_{2}, \ldots$ of words over $\Sigma$ the set

$$
\left|\sigma_{1}, \sigma_{2}, \ldots\right| \triangleq\left\{\begin{array}{cc}
\left\{\sigma_{1} \sigma_{2} \ldots\right\} \times \Sigma^{\omega} & \text { if } \sigma_{1} \sigma_{2} \ldots \text { is a finite word } \\
\left\{\sigma_{1} \sigma_{2} \ldots\right\} & \text { otherwise. }
\end{array}\right.
$$


Proof. At first let us note that we may assume $\beta=\beta^{*} \cdot \beta$. This is a consequence of the fact that $\beta^{\omega}=\left(\beta^{*} \cdot \beta\right)^{\omega 6}$. Restated, this condition means that for any $i, j, k \in n$ we have:

$$
i \stackrel{\sigma}{\rightarrow}_{\beta} j{\stackrel{\sigma_{2}}{\rightarrow}}_{\beta} k \Longrightarrow i{\stackrel{\sigma_{1} \sigma_{2}}{\rightarrow}}_{\beta} k .
$$

Let $\beta^{o}: n \rightarrow 0=n \rightarrow \mathcal{P}\left(\Sigma^{\omega}\right)$ be a map whose value $\beta^{o}(i)$ is given in terms of the right hand side of the equality 3.1. Observe that this map satisfies $\beta^{o}=\beta^{o} \cdot \beta$ in $\operatorname{LTS}^{\omega}$. This follows directly from the definition of $\beta^{o}$ and the formula for the composition in $\mathcal{K l}\left(\mathcal{P}\left(\Sigma^{*} \times \mathcal{I} d+\Sigma^{\omega}\right)\right)$. Thus, $\beta^{o} \leq \beta^{\omega}$. Now, by contradiction, if $\beta^{o}<\beta^{\omega}$ then there is $i$ and $v \in \Sigma^{\omega}$ such that $v \in \beta^{\omega}(i)$ and $v \notin \beta^{o}(i)$. Hence, in particular, this means that there is an infinite sequence of transitions $i \stackrel{\sigma_{1}}{\rightarrow} i_{1} \stackrel{\sigma_{2}}{\rightarrow} i_{2} \ldots$ in $\beta$ which starts at $i$. If there was no such sequence, this would mean that $\beta^{o}(i)=\varnothing=\beta^{\omega}(i)$ which cannot hold. Since $\beta^{\omega}=\beta^{\omega} \cdot \beta$, there is a state $i_{1}$ and $\sigma_{1} \in \Sigma^{*}, v_{1} \in \Sigma^{\omega}$ such that $i \stackrel{\sigma}{\rightarrow}_{\beta} i_{1}$ and $v_{1} \in \beta^{\omega}\left(i_{1}\right)$ with $v=\sigma_{1} v_{1}$. Note that we may assume $\sigma_{1} \neq \varepsilon$ as there has to be a prefix $\sigma_{1} \neq \varepsilon$ of $v=\sigma_{1} v_{1}$ with $i \stackrel{\sigma_{子}}{\rightarrow} i_{1}$ for some state $i_{1}$ with $v_{1} \in \beta^{\omega}\left(i_{1}\right)$. If it was otherwise, then by 3.2 we would have an infinite sequence $i \stackrel{\varepsilon}{\rightarrow} i_{1} \stackrel{\varepsilon}{\rightarrow} i_{2} \stackrel{\varepsilon}{\rightarrow} \ldots$ yielding $\beta^{\omega}(i)=\Sigma^{\omega}=\beta^{o}(i)$ which contradicts our assumptions. Hence, if $i \stackrel{\sigma_{f}}{\rightarrow} i_{1}$ for $\sigma_{1} \neq \varepsilon$ and $v_{1} \in \beta^{\omega}\left(i_{1}\right)$ then we also have $v_{1} \notin \beta^{o}\left(i_{1}\right)$. By inductively repeating this argument we get a sequence $i \stackrel{\sigma_{7}}{\rightarrow} i_{1} \stackrel{\sigma_{2}}{\rightarrow} i_{2} \ldots$ in $\beta$ such that $\sigma_{k} \neq \varepsilon$ and $v=\sigma_{1} \sigma_{2} \ldots$ Thus, by the definition of $\beta^{o}$ we also get $v \in \beta^{o}(i)$ which is a contradiction.

3.1.3. Büchi acceptance condition. Before we spell out the recipe of how to extract $\omega$-language of any state in the automaton $(\alpha, \mathfrak{F})$ in terms of $(-)^{*},(-)^{\omega}$ and the composition in LTS $^{\omega}$, we need one last ingredient. Let us define $\alpha^{+} \triangleq \alpha^{*} \cdot \alpha$ and note

$$
\alpha^{+}(i)=\left\{(\sigma, j) \mid i \stackrel{a_{1}}{\rightarrow} i_{1} \ldots \stackrel{a_{k}}{\rightarrow} i_{k} \text { in } \alpha \text { and } \sigma=a_{1} \ldots a_{k} \text { for } k \geq 1\right\} .
$$

Hence, $\mathfrak{f}_{\mathfrak{F}} \cdot \alpha^{+}$viewed as an endomorphism in $\mathrm{LTS}^{\omega}$ is given by $\mathfrak{f}_{\mathfrak{F}} \cdot \alpha^{+}: n-\circ \rightarrow n=n \rightarrow$ $\mathcal{P}\left(\Sigma^{*} \times n+\Sigma^{\omega}\right)$ where:

$$
\mathfrak{f}_{\mathfrak{F}} \cdot \alpha^{+}(i)=\left\{(\sigma, j) \mid i \stackrel{\sigma}{\rightarrow} j \text { in } \alpha^{+} \text {and } j \in \mathfrak{F}\right\} .
$$

Finally, consider the following map in $\mathrm{LTS}^{\omega}$ :

$$
\left(\mathfrak{f}_{\mathfrak{F}} \cdot \alpha^{+}\right)^{\omega}: n \multimap 0=n \rightarrow \mathcal{P}\left(\Sigma^{\omega}\right)
$$

By Proposition 3.1, the map $\left(\mathfrak{f}_{\mathfrak{F}} \cdot \alpha^{+}\right)^{\omega}$ satisfies:

$$
\left(\mathfrak{f}_{\mathfrak{F}} \cdot \alpha^{+}\right)^{\omega}(i)=\text { the } \omega \text {-language of } i \text { in the Büchi automaton represented by }(\alpha, \mathfrak{F}) \text {. }
$$

The above statement suggests a general approach towards modelling ( $\omega$-)behaviours of abstract (coalgebraic) automata which we will develop in the sections to come.

\footnotetext{
${ }^{6}$ This identity is proven in Lemma 5.4 in the general setting of ordered theories equipped with $(-)^{*}=$ $\mu x$. (id $\vee x \cdot(-))$ and $(-)^{\omega}=\nu x . x \cdot(-)$ satisfying additional conditions that, in particular, hold for LTS L $^{\omega}$ Hence, we refer the reader to Section 5 for the general proof of the statement.
} 

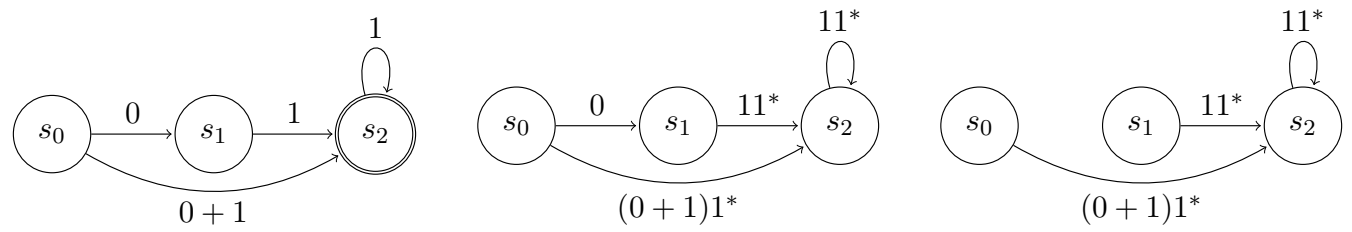

Figure 4: An automaton $(\alpha, \mathfrak{F})$ and the maps $\alpha^{+}$and $\mathfrak{f}_{\mathfrak{F}} \cdot \alpha^{+}$.

3.2. Tree automata. Let us now focus our attention on tree automata and their behaviour. Just like in the previous subsection we may consider automata over the state space $n$. Moreover, as before, we also encode any tree automaton $(n, \Sigma, \delta, \mathfrak{F})$ as a pair $(\alpha: n \rightarrow$ $\mathcal{P}(\Sigma \times n \times n), \mathfrak{F})$. Since $\mathcal{P}(\Sigma \times n \times n) \subseteq \mathcal{P} T_{\Sigma} n$, the transition map $\alpha$ can be viewed as $\alpha: n \rightarrow \mathcal{P} T_{\Sigma} n$ (i.e. as an endomorphism in the Kleisli category for the monad $\mathcal{P} T_{\Sigma}$ or, equivalently, as an endomorphism in the theory TTS ${ }^{\omega}$ ). The hom-sets of the Kleisli category for $\mathcal{P} T_{\Sigma}$ and its full subcategory TTS $^{\omega}$ admit ordering in which we can define $\beta^{*}, \beta^{+}$and $\beta^{\omega}$ for any $\beta: n \multimap \rightarrow n$ as in the previous subsection. Not surpisingly, if we now compute $! \cdot \mathfrak{f}_{\mathfrak{F}} \cdot \alpha^{*}$ and $\left(\mathfrak{f}_{\mathfrak{F}} \cdot \alpha^{+}\right)^{\omega}$ in $\operatorname{TTS}^{\omega}$ we exactly get the following ${ }^{7}$.

Proposition 3.2. For any $i \in n$ we have:

$$
\begin{aligned}
! \cdot \mathfrak{f}_{\mathfrak{F}} \cdot \alpha^{*}(i) & =\text { the set of finite trees recognized by } i \text { in }(n, \Sigma, \delta, \mathfrak{F}), \\
\left(\mathfrak{f}_{\mathfrak{F}} \cdot \alpha^{+}\right)^{\omega}(i) & =\text { the set of inifinite trees recognized by } i \text { in }(n, \Sigma, \delta, \mathfrak{F}) .
\end{aligned}
$$

3.3. Kleene theorems, categorically. The purpose of this subsection is to restate classical Kleene theorems from Subsection 2.1 on the categorical level for LTS $^{\omega}$ and TTS ${ }^{\omega}$. Before we do this let us elaborate more on why we choose our setting to be systems whose type is a monad.

Remark 3.3. As the examples of non-deterministic (tree) automata studied in the previous subsection do not admit silent moves, the reader may get an impression that the need for categorical modelling of infinite behaviour for systems with silent steps is not sufficiently justified. To add to this, although $\varepsilon$-moves are a standard feature of automata whenever it comes to their finitary languages, invisible moves rarely occur in practice in the classical literature on the infinite behaviour (with BAC) (see e.g. [PP04]). However, as already mentioned in the introduction, incorporation of silent moves should be viewed as a byproduct of our paper's framework, not its main purpose. The main aim is to build a simple bridge between syntax and semantics of regular and $\omega$-regular behaviours in the form of generic Kleene theorems. Once we embed our systems into systems whose type is a monad $T$, the syntax arises from the algebraicity of $T$ and the semantics is provided by automata whose transition maps are certain $T$-coalgebras. This also allows us to abstract away from several "unnecessary" details and focus on core properties.

As witnessed in Subsection 2.1, Kleene theorems for tree automata were slightly more involved than their classical counterparts for non-deterministic automata. The reason for this is simple: non-deterministic automata accept sequential data types. Whenever we deal

\footnotetext{
${ }^{7}$ The proof of Proposition 3.2 is intensionally omitted as it goes along the lines of the series of statements made in Subsection 3.1 for non-deterministic automata.
} 
with non-sequential data, e.g. trees, the set of $(\omega$-)regular languages is expected to be closed under a more complex type of composition, i.e. the composition of regular languages with multiple variables [GTW02, PP04]. Hence, if we aim at categorical statements generalizing theory from Subsection 2.1 then we should expect a slightly more involved formulation to be our point of reference. Hence, we start with presenting a categorical perspective of Kleene theorems for tree automata first.

3.3.1. Tree automata. The Kleene theorems for regular and $\omega$-regular input from Subsection 2.2.4 are equivalent to the following proposition.

Proposition 3.4. Let $\mathfrak{R e g}$ and $\omega \mathfrak{R e g}$ be defined as in Subsection 2.2.4. Let $\mathfrak{R a t}$ be the smallest subtheory of TTS $^{\omega}$ such that:

(a) it contains all maps of the form $n \rightarrow \mathcal{P}\left(\Sigma_{\varepsilon} \times n \times n\right) \hookrightarrow \mathcal{P} T_{\Sigma} n$,

(b) is closed under finite suprema,

(c) its endomorphisms are closed under $(-)^{*}$.

Then the hom-set $\mathfrak{R a t}(1,1)$ of the theory $\mathfrak{R a t}$ equals to $\mathfrak{R e g}$. Moreover, the set $\omega \mathfrak{R e g}$ of $\omega$-regular languages for tree automata satisfies $\left\{r^{\omega} \cdot s \mid r \in \mathfrak{R a t}(n, n), s \in \mathfrak{R a t}(1, n)\right\}=\omega \mathfrak{R e g}$.

3.3.2. Non-deterministic automata. The formulation of Proposition 3.4 allows us to instantiate it for non-deterministic automata. A simple verification proves that the following holds.

Proposition 3.5. Let $\mathfrak{R a t}$ be the smallest subtheory of $\mathrm{LTS}^{\omega}$ such that:

(a) it contains all maps of the form $n \rightarrow \mathcal{P}\left(\Sigma_{\varepsilon} \times n\right) \hookrightarrow \mathcal{P}\left(\Sigma^{*} \times n+\Sigma^{\omega}\right)$,

(b) it is closed under finite suprema,

(c) its endomorphisms are closed under $(-)^{*}$.

Then the hom-set $\mathfrak{R a t}(1,1)$ of the theory $\mathfrak{R a t}$ equals to:

$$
\left\{r: 1 \rightarrow \mathcal{P}\left(\Sigma^{*} \times 1\right) \subseteq \mathcal{P}\left(\Sigma^{*} \times 1+\Sigma^{\omega}\right) \mid r(1)=R \times\{1\} \text { where } R \subseteq \Sigma^{*} \text { is regular }\right\} .
$$

Additionally, the set $\omega \mathfrak{R e g} \triangleq\left\{r: 1 \multimap 0=1 \rightarrow \mathcal{P}\left(\Sigma^{\omega}\right) \mid r(1)\right.$ is $\omega$-regular $\}$ satisfies:

$$
\left\{r^{\omega} \cdot s: 1 \multimap 0=1 \rightarrow \mathcal{P}\left(\Sigma^{\omega}\right) \mid r \in \mathfrak{R a t}(n, n), s \in \mathfrak{R a t}(1, n)\right\}=\omega \mathfrak{R e g} .
$$

3.4. Beyond tree automata. There are variants of non-deterministic (Büchi) automata that accept other types of input (e.g. arbitrary finitely-branching trees, see e.g. [USH16]). In general, given a functor $F$ : Set $\rightarrow$ Set we define a non-deterministic (Büchi) $F$-automaton as a pair $(\alpha, \mathfrak{F})$, where $\alpha: n \rightarrow \mathcal{P} F n$ and $\mathfrak{F} \subseteq n$. A natural question that arises is the following: are we able to build a general categorical setting in which we can reason about the (in)finite behaviour of systems for arbitrary non-deterministic Büchi $F$-automata (or even more generally, for systems of the type $T F$ for a monad $T)$ ? If so, then is it possible to generalize the Kleene theorem for $(\omega-)$ regular languages to a coalgebraic level? We will answer these questions positively in the next sections. 


\section{Monads for (in)Finite Behaviour}

Given a monad $T$ and an endofunctor $F$ on a common category, the purpose of this section is to provide a construction of a monad $T F^{\infty}$ which extends the functor $T F$. The monad $T F^{\infty}$ will prove itself sufficient to model the combination of finite and infinite behaviour (akin to the monad $\mathcal{P}\left(\Sigma^{*} \times \mathcal{I} d+\Sigma^{\omega}\right)$ for the functor $\mathcal{P}(\Sigma \times \mathcal{I} d)$, or $\mathcal{P} T_{\Sigma}$ for $\left.\mathcal{P}\left(\Sigma \times \mathcal{I} d^{2}\right)\right)$.

At first we list all assumptions required in the remainder of this section. Later, in Subsection 4.2, we revisit the construction of the $\operatorname{monad} T F^{*}$ from [Bre15]. Finally, we give a description of $T F^{\infty}$.

Assumption 4.1. Let $\mathrm{C}$ be a category which admits binary coproducts. We denote the coproduct operator by + and the coprojection into the first and the second component of a coproduct by inl and inr respectively. Moreover, let $\mathrm{F}: \mathrm{C} \rightarrow \mathrm{C}$ be a functor. In what follows, in this section we additionally assume:

(i) $(T, \mu, \eta)$ is a monad on $\mathrm{C}$ and $F: \mathrm{C} \rightarrow \mathrm{C}$ lifts to $\mathcal{K} l(T)$ via a distributive law $\lambda: F T \Longrightarrow T F$,

(ii) there is an initial $F(-)+X$-algebra for any object $X$ and a terminal $F$-coalgebra $\zeta: F^{\omega} \rightarrow F F^{\omega}$

(iii) the category $\operatorname{Alg}(F)$ of $F$-algebras admits binary coproducts (with the coproduct operator denoted by $\oplus)$.

4.1. Preliminaries. The initial $F(-)+X$-algebra $i_{X}: F F^{*} X+X \rightarrow F^{*} X$ yields the free $F$-algebra over $X$ given by $i_{X} \circ \mathrm{inl}: F F^{*} X \rightarrow F^{*} X$. Hence, by our assumptions we have an adjoint situation $C \rightleftarrows \operatorname{Alg}(F)$, where the left adjoint is the free algebra functor which assigns to any object $X$ the free algebra $i_{X} \circ$ inl over it. The right adjoint is the forgetful functor which assigns to any $F$-algebra its carrier and is the identity on morphisms. The adjunction yields the monad $F^{*}: \mathrm{C} \rightarrow \mathrm{C}$ which assigns to any object $X$ the carrier of the free $F$-algebra over $X$.

Example 4.2. For any set $\Sigma$ and $X$ the initial $\Sigma \times \mathcal{I} d+X$-algebra is given by the morphism $i_{X}: \Sigma \times \Sigma^{*} \times X+X \rightarrow \Sigma^{*} \times X$, where

$$
i_{X}(a,(\sigma, x))=(a \sigma, x) \text { and } i_{X}(x)=(\varepsilon, x) .
$$

4.1.1. Bloom algebras. The purpose of this subsection is to recall basic definitions and properties of Bloom $F$-algebras [AHM14] whose free algebras yield a monad $F^{\infty}$ on $C$ which extends the functor $F$. This will allow us to embed systems of the type $X \rightarrow T F X$ to systems of the type $X \rightarrow T F^{\infty} X$ and discuss their (in)finite behaviour in the latter context.

A pair $\left(a: F A \rightarrow A,(-)^{\dagger}\right)$ is called a Bloom $F$-algebra provided that any $F$-coalgebra $e: X \rightarrow F X$ yields the map $e^{\dagger}: X \rightarrow A$ which satisfies:

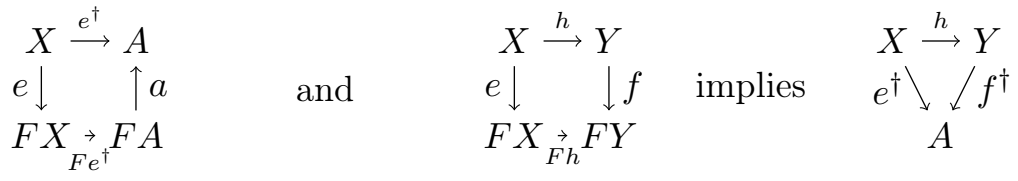


A homomorphism from a Bloom algebra $\left(a: F A \rightarrow A,(-)^{\dagger}\right)$ to a Bloom algebra $(b: F B \rightarrow$ $\left.B,(-)^{\ddagger}\right)$ is a map $h: A \rightarrow B$ which is an $F$-algebra homomorphism from $a$ to $b$, which additionally preserves the dagger, i.e. $e^{\dagger} \circ h=e^{\ddagger}$. The category of Bloom algebras and homomorphisms between them is denoted by $\operatorname{Alg}_{B}(F)$. We have the following theorem.

Theorem 4.3 [AHM14]. The pair $\left(\zeta^{-1}: F F^{\omega} \rightarrow F^{\omega}\right.$, [[-]]), where [[-]] assigns to $e: X \rightarrow$ $F X$ the unique coalgebra homomorphism $[[e]]: X \rightarrow F^{\omega}$ between e and $\zeta$, is an initial object in $\operatorname{Alg}_{B}(F)$. Moreover, the F-algebra coproduct

$$
\left(i_{X} \circ \text { inl }: F F^{*} X \rightarrow F^{*} X\right) \oplus\left(\zeta^{-1}: F F^{\omega} \rightarrow F^{\omega}\right)
$$

is the free Bloom algebra over $X$.

Remark 4.4. Let $F^{\infty}: \mathrm{C} \rightarrow \mathrm{C}$ be defined as the composition of the left and right adjoints $\mathrm{C} \rightleftarrows \operatorname{Alg}_{B}(F)$, where the left adjoint is the free Bloom algebra functor and the right adjoint is the forgetful functor. The functor $F^{\infty}$ carries a monadic structure which extends $F^{*}$. Indeed, by Theorem 4.3, the monad $F^{*}$ is a submonad of $F^{\infty}$ (via the transformation induced by the coprojection into the first component of $i_{X} \circ \operatorname{inl} \oplus \zeta^{-1}$ in $\left.\operatorname{Alg}(F)\right)$. The formula for the free Bloom algebra from the above theorem indicates that $F^{\infty}$ is a natural extension of $F^{*}$ encompassing infinite behaviours of the final $F$-coalgebra. By abusing the notation slightly, we can write

$$
F^{\infty}=F^{*} \oplus F^{\omega} .
$$

The functor $F_{\varepsilon}$ is a subfunctor of $F^{*}$ [Bre15, Lemma 4.12] and hence, by the above, also of $F^{\infty}$. In the following sections this will let us turn any coalgebra $X \rightarrow T F X$ or $X \rightarrow T F_{\varepsilon} X$ into a system $X \rightarrow T F^{\infty} X$ and, by doing so, allow us to model their (in)finite behaviour.

Example 4.5. The terminal $\Sigma \times \mathcal{I} d$-coalgebra is

$$
\zeta: \Sigma^{\omega} \rightarrow \Sigma \times \Sigma^{\omega} ; a_{1} a_{2} \ldots \mapsto\left(a_{1}, a_{2} a_{3} \ldots\right) .
$$

The coproduct of $a: \Sigma \times A \rightarrow A$ and $b: \Sigma \times B \rightarrow B$ in $\operatorname{Alg}(F)$ is

$$
a \oplus b: \Sigma \times(A+B) \rightarrow A+B ;(\sigma, x) \mapsto \begin{cases}a(\sigma, x) & \text { if } x \in A \\ b(\sigma, x) & \text { otherwise. }\end{cases}
$$

Hence, the free Bloom algebra over $X$ is a map $\Sigma \times\left(\Sigma^{*} \times X+\Sigma^{\omega}\right) \rightarrow \Sigma^{*} \times X+\Sigma^{\omega}$ explicitly given by: $(a,(\sigma, x)) \mapsto(a \sigma, x)$ and $\left(a, a_{1} a_{2} \ldots\right) \mapsto a a_{1} a_{2} \ldots$

Let $\left(a: F A \rightarrow A,(-)^{\dagger}\right)$ be a Bloom algebra, $b: F B \rightarrow B$ an $F$ algebra and $h: A \rightarrow B$ a homomorphism between $F$-algebras $a$ and $b$. Then there is a unique assignment $(-)^{\ddagger}$ which turns $\left(b: F B \rightarrow B,(-)^{\ddagger}\right)$ into a Bloom algebra and $h$ into a Bloom algebra homomorphism and it is defined as follows [AHM14]: for $e: X \rightarrow F X$ the map $e^{\ddagger}: X \rightarrow B$ is $e^{\ddagger} \triangleq h \circ e^{\dagger}$.

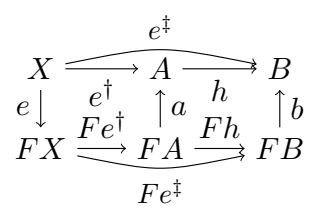

4.2. Lifting monads to algebras. Take an $F$-algebra $a: F A \rightarrow A$ and define $\bar{T}(a) \triangleq$ $F T A \stackrel{\lambda_{A}}{\rightarrow} T F A \stackrel{T a}{\rightarrow} T A$. If $h: A \rightarrow B$ is a homomorphism of algebras $a$ and $b: F B \rightarrow B$ we put $\bar{T}(h)=T(h)$. In this case $\bar{T}: \operatorname{Alg}(F) \rightarrow \operatorname{Alg}(F)$ is a functor for which the morphism $\eta_{A}: A \rightarrow T A$ is an $F$-algebra homomorphism from $a: F A \rightarrow A$ to $\bar{T}(a): F T A \rightarrow T A$. Moreover, $\mu_{A}: T^{2} A \rightarrow T A$ is a homomorphism from $\bar{T}^{2}(a)$ to $\bar{T}(a)$ (see [Bec69] for details). A direct consequence of this construction is the following. 
Theorem 4.6 [Bec69]. The triple $(\bar{T}, \bar{\mu}, \bar{\eta})$, where for $a: F A \rightarrow A$ we put

$$
\bar{\mu}_{a}: \bar{T}^{2}(a) \rightarrow \bar{T}(a) ; \bar{\mu}_{a}=\mu_{A} \text { and } \bar{\eta}_{a}: a \rightarrow \bar{T}(a) ; \bar{\eta}_{a}=\eta_{A}
$$

is a monad on $\operatorname{Alg}(F)$.

The above theorem together with the assumption of existence of an arbitrary free $F$-algebra in $\operatorname{Alg}(F)$ leads to a pair of adjoint situations captured by the diagram on the right. Since the composition of adjunctions is an adjunction this yields a monadic structure

$$
C_{\ltimes} \rightleftharpoons \lg (F) \underset{\kappa}{\perp} \mathcal{K} l(\bar{T})
$$
on the functor $T F^{*}: \mathrm{C} \rightarrow \mathrm{C}$.

Example 4.7. An example of this phenomenon is given by the $\operatorname{monad} \mathcal{P}\left(\Sigma^{*} \times \mathcal{I} d\right)$ from Example 2.6 where in the above we set $T=\mathcal{P}$ and $F=\Sigma \times \mathcal{I} d$. This monad has already been described e.g. in [Bre15], but it arose as a consequence of the composition of a different pair of adjunctions.

4.2.1. Monads on Bloom algebras. Above we gave a recipe for a general construction of a monadic structure on the functor $T F^{*}$. As witnessed in [Bre15, BMSZ15], this monad is suitable to model coalgebras and their weak bisimulations and weak finite trace semantics (i.e. their finite behaviour). Our primary interest is in modelling infinite behaviour and this monad proves itself insufficient. The purpose of this subsection is to show how to tweak the middle category from the pair of adjunctions in pictured in the diagram above so that the monad obtained from the composition of two adjunctions is suitable for our needs.

Let $\left(a: F A \rightarrow A,(-)^{\dagger}\right)$ be a Bloom algebra and define

$$
\bar{T}_{B}\left(\left(a: F A \rightarrow A,(-)^{\dagger}\right)\right) \triangleq\left(\bar{T}(a): F T A \rightarrow T A,(-)^{\ddagger}\right),
$$

where for any $e: X \rightarrow F X$ the map $e^{\ddagger}$ is given by $\eta_{A} \circ e^{\dagger}$. Since $\eta_{A}: A \rightarrow T A$ is a homomorphism between $a: F A \rightarrow A$ and $\bar{T}(a): F T A \rightarrow T A$ the pair $\left(\bar{T}(a),(-)^{\ddagger}\right)$ is a Bloom algebra. For a pair of Bloom algebras $\left(a: F A \rightarrow A,(-)^{\dagger}\right)$ and $\left(b: F B \rightarrow B,(-)^{\ddagger}\right)$ and a Bloom algebra homomorphism $h: A \rightarrow B$ between them put $\bar{T}_{B}(h)=T(h)$. This defines a functor $\bar{T}_{B}: \operatorname{Alg}_{B}(F) \rightarrow \operatorname{Alg}_{B}(F)$. Analogously to the previous subsection we have the following direct consequence of the construction.

Theorem 4.8. The triple $\left(\bar{T}_{B}, \bar{\mu}^{B}, \bar{\eta}^{B}\right)$ is a monad on $\operatorname{Alg}_{B}(F)$, where for any Bloom algebra $\left(a: F A \rightarrow A,(-)^{\dagger}\right)$ the $\left(a,(-)^{\dagger}\right)$-components of the transformations $\bar{\mu}^{B}$ and $\bar{\eta}^{B}$ are

$$
\begin{aligned}
& \bar{\mu}_{\left(a,(-)^{\dagger}\right)}^{B}: \bar{T}_{B}^{2}\left(a,(-)^{\dagger}\right) \rightarrow \bar{T}_{B}\left(a,(-)^{\dagger}\right) ; \quad \bar{\mu}_{\left(a,(-)^{\dagger}\right)}^{B}=\mu_{A} \text { and } \\
& \bar{\eta}_{\left(a,(-)^{\dagger}\right)}^{B}:\left(a,(-)^{\dagger}\right) \rightarrow \bar{T}_{B}\left(a,(-)^{\dagger}\right) \text { with } \bar{\eta}_{\left(a,(-)^{\dagger}\right)}^{B}=\eta_{A} .
\end{aligned}
$$

Hence, we obtain two adjoint situations captured in the diagram below. These adjunctions impose a monadic structure on the functor $T F^{\infty}: \mathrm{C} \rightarrow \mathrm{C}$. The monad $\mathcal{P}\left(\Sigma^{*} \times \mathcal{I} d+\Sigma^{\omega}\right)$ from Example 2.7 arises from the composition of the above adjoint

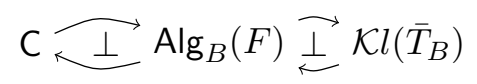
situations (see also Example 4.5).

Example 4.9. Let $F=\Sigma \times \mathcal{I} d^{2}$. This functor lifts to $\mathcal{K} l(\mathcal{P})$ [HJS07] and, up to isomorphism, $F^{\infty}=T_{\Sigma}$ is a functor which assigns to any set $X$ the set of complete binary trees (i.e. every node has either two children or no children) with inner nodes taking values in $\Sigma$ and finitely many leaves, all taken from $X$ [AHM14] (see Subsection 2.1). This yields a monadic structure on $\mathcal{P} F^{\infty}=\mathcal{P} T_{\Sigma}$ defined in Example 2.8. 
The above example can be easily generalized. Indeed, if $T$ is a commutative Set-based monad then any polynomial functor ${ }^{8} F:$ Set $\rightarrow$ Set lifts to $\mathcal{K} l(T)$ [HJS07]. If it admits all free $F$-algebras and the final $F$-coalgebra then Assumption 4.1 holds for $T$ and $F$ yielding the monad $T F^{\infty}$.

\section{Abstract automata and their behaviour}

The purpose of this section is to generalize the concepts from Section 3 to an arbitrary Kleisli category with a suitable ordering. In other words, given a Set-monad $T$, we define a $T$-automaton, its finite behaviour, its infinite behaviour with $\mathrm{BAC}$ and provide generic Kleene theorems for $T$.

Let $(T, \mu, \eta)$ be a Set-based monad. Since we will often consider the Lawvere theory $\mathbb{T}$ associated with it, recall that its objects are sets given by $n=\{1, \ldots, n\}$ for $n=0,1, \ldots$. We start with the definition of a $T$-automaton.

Definition 5.1. A T-automaton or simply automaton is a pair $(\alpha, \mathfrak{F})$, where $\alpha: X \rightarrow X=$ $X \rightarrow T X$ is a $T$-coalgebra called transition morphism and $\mathfrak{F} \subseteq X$.

Assumption 5.2. In order to define finite and infinite behaviour of $(\alpha, \mathfrak{F})$ and reason about it we require the Kleisli category for $T$ to satisfy more assumptions. In this section we assume that:

(A) $\mathcal{K} l(T)$ is order enriched with a pointwise induced order,

(B) it is $\omega$ Cpo-enriched,

(C) it is left distributive,

(D) its hom-sets are complete lattices.

At first let us note that, in the light of Remark 2.9, by (A) we get:

- right distributivity w.r.t. the base morphisms and

- cotupling order preservation.

Additionally, since the Kleisli category for $T$ is left distributive we have:

- the bottoms $\perp_{X, Y} \in \mathcal{K} l(T)(X, Y)$ satisfy $g \cdot \perp_{X, Y}=\perp_{X, Z}$ for any $g: Y \multimap Z$.

The axioms (A)-(C) guarantee that the saturation $\alpha \mapsto \alpha^{*}$ is computable in $\omega$-steps and is expressive enough. For a given $\alpha: X \rightarrow X=X \rightarrow T X$ we can define maps $\alpha^{*}, \alpha^{+}$: $X \multimap X=X \rightarrow T X$ :

$$
\alpha^{*} \triangleq \mu x .(\mathrm{id} \vee x \cdot \alpha) \text { and } \alpha^{+} \triangleq \alpha^{*} \cdot \alpha .
$$

The operator $\alpha \mapsto \alpha^{*}$ was thoroughly studied in [Bre14, Bre15, BMP15, BP16] in the context of coalgebraic weak bisimulation. Its definition does not require a complete lattice order. See loc. cit. for a discussion.

Assumption (D) allows us to define the greatest fixpoint of the map $x \mapsto x \cdot \alpha$. Indeed for any $\alpha: X \multimap X=X \rightarrow T X$ put $\alpha^{\omega}: X \multimap 0=X \rightarrow T 0$ to be:

$$
\alpha^{\omega} \triangleq \bigwedge_{\kappa \in \text { Ord }}(x \mapsto x \cdot \alpha)^{\kappa}(\top)
$$

where $\top: X \multimap 0=X \rightarrow T 0$ is the greatest element of $\mathcal{K} l(T)(X, 0)$ and

$$
(x \mapsto x \cdot \alpha)^{\kappa}:(X \multimap \rightarrow 0) \rightarrow(X \multimap \rightarrow 0)
$$

\footnotetext{
${ }^{8} \mathrm{~A}$ polynomial functor is a functor defined by the grammar $F \triangleq \Sigma \in$ Set $|\mathcal{I} d| F \times F \mid \sum F$ [HJS07].
} 
is defined in terms of the transfinite induction by

$$
(x \mapsto x \cdot \alpha)^{\kappa+1} \triangleq(x \mapsto x \cdot \alpha) \circ(x \mapsto x \cdot \alpha)^{\kappa}
$$

for a successor ordinal $\kappa+1$ and the ordinary map composition operator $\circ$, and $(x \mapsto$ $x \cdot \alpha)^{\kappa} \triangleq \bigwedge_{\lambda<\kappa}(x \mapsto x \cdot \alpha)^{\lambda}$ for a limit ordinal $\kappa$. By the Tarski-Knaster theorem, the map $\alpha^{\omega}: X \multimap \rightarrow 0=X \rightarrow T 0$ is the greatest fixpoint of the assignment $x \mapsto x \cdot \alpha$.

Example 5.3. The Kleisli categories for the monads from Example 2.10 satisfy (A)-(D). Section 6 presents one more example of a Kleisli category that fits the setting in the context of probabilistic automata.

Before we present the definition of finite and infinite behaviour of automata we need one more technical result.

Lemma 5.4. For any $\alpha, \beta: X \multimap \rightarrow=X \rightarrow T X$ we have:

(1) $\alpha^{*}=\bigvee_{n}(\mathrm{id} \vee \alpha)^{n}$,

(2) $\mathrm{id}^{*}=\mathrm{id}$, id $\leq \alpha^{*}$ and $\alpha^{*} \cdot \alpha^{*}=\alpha^{*}$,

(3) $(\alpha \cdot \beta)^{\omega}=(\beta \cdot \alpha)^{\omega} \cdot \beta$,

(4) $\left(\alpha^{n}\right)^{\omega}=\alpha^{\omega}$ for any $n>0$,

(5) $\alpha^{\omega}=\left(\alpha^{+}\right)^{\omega}$.

Proof. The proof of 1 and 2 can be found in [Bre15, Lemma 5.1 and Theorem 5.7]. To see 3 holds, i.e. $(\alpha \cdot \beta)^{\omega}=(\beta \cdot \alpha)^{\omega} \cdot \beta$ note that $(\beta \cdot \alpha)^{\omega} \cdot \beta$ is a fixpoint of $x \mapsto x \cdot \alpha \cdot \beta$ and hence $(\beta \cdot \alpha)^{\omega} \cdot \beta \leq(\alpha \cdot \beta)^{\omega}$. By a similar argument we show $(\alpha \cdot \beta)^{\omega} \cdot \alpha \leq(\beta \cdot \alpha)^{\omega}$. Thus,

$$
(\beta \cdot \alpha)^{\omega}=(\beta \cdot \alpha)^{\omega} \cdot \beta \cdot \alpha \leq(\alpha \cdot \beta)^{\omega} \cdot \alpha \leq(\beta \cdot \alpha)^{\omega} .
$$

To prove $\left(\alpha^{n}\right)^{\omega}=\alpha^{\omega}$ note that by 3 we have $\left(\alpha^{n}\right)^{\omega}=\left(\alpha^{n-1} \cdot \alpha\right)^{\omega}=\left(\alpha \cdot \alpha^{n-1}\right)^{\omega} \cdot \alpha$. Hence, $\left(\alpha^{n}\right)^{\omega} \leq \alpha^{\omega}$. Moreover, since $\alpha^{\omega} \cdot \alpha^{n}=\alpha^{\omega} \cdot \alpha^{n-1}=\cdots=\alpha^{\omega}$ we get the converse inequality, i.e. $\alpha^{\omega} \leq\left(\alpha^{n}\right)^{\omega}$. This proves the assertion.

Finally, note that by monotonicity of $(-)^{\omega}$ since $\alpha \leq \alpha^{*} \cdot \alpha=\alpha^{+}$we have $\alpha^{\omega} \leq\left(\alpha^{+}\right)^{\omega}$. Moreover,

$$
\begin{aligned}
& \left(\alpha^{+}\right)^{\omega}=\left(\alpha^{*} \cdot \alpha\right)^{\omega} \leq\left(\alpha^{*} \cdot \alpha\right)^{\omega} \cdot(\operatorname{id} \vee \alpha)=\left(\alpha^{*} \cdot \alpha\right)^{\omega} \vee\left(\alpha^{*} \cdot \alpha\right)^{\omega} \cdot \alpha \leq \\
& \left(\alpha^{*} \cdot \alpha\right)^{\omega} \vee\left(\alpha^{*} \cdot \alpha\right)^{\omega} \cdot \alpha^{*} \cdot \alpha=\left(\alpha^{*} \cdot \alpha\right)^{\omega} \vee\left(\alpha^{*} \cdot \alpha\right)^{\omega}=\left(\alpha^{+}\right)^{\omega}
\end{aligned}
$$

Hence, by induction we prove that $\left(\alpha^{+}\right)^{\omega} \cdot(\text { id } \vee \alpha)^{i}=\left(\alpha^{+}\right)^{\omega}$. By the fact that our theory is $\omega$ Cpo-enriched we get:

$$
\left(\alpha^{+}\right)^{\omega} \cdot \alpha^{*}=\left(\alpha^{+}\right)^{\omega} \cdot \bigvee_{i}(\operatorname{id} \vee \alpha)^{i}=\bigvee_{i}\left(\alpha^{+}\right)^{\omega} \cdot(\operatorname{id} \vee \alpha)^{i}=\left(\alpha^{+}\right)^{\omega}
$$

This proves that $\left(\alpha^{+}\right)^{\omega}$ satisfies $\left(\alpha^{+}\right)^{\omega} \cdot \alpha^{*} \cdot \alpha=\left(\alpha^{+}\right)^{\omega} \cdot \alpha$. Thus, $\left(\alpha^{+}\right)^{\omega} \leq \alpha^{\omega}$ which completes the proof.

5.1. Finite and infinite behaviour. The purpose of this subsection is to present the definitions of the finite and infinite behaviour with BAC for T-automata. Let $(\alpha: X \rightarrow$ $T X, \mathfrak{F} \subseteq X$ ) be a $T$-automaton. Before we start, let us first encode the set $\mathfrak{F}$ of accepting states in terms of an endomorphism $\mathfrak{f}_{\mathfrak{F}}: X \multimap X=X \rightarrow T X$ by:

$$
\mathfrak{f}_{\mathfrak{F}}(x)=\left\{\begin{array}{cc}
\eta_{X}(x) & \text { if } x \in \mathfrak{F}, \\
\perp & \text { otherwise }
\end{array} \text { for any } x \in X\right.
$$

where $\perp$ denotes the bottom element of the poset $T X$. 
Definition 5.5. Finite and $\omega$-behaviour of the automaton $(\alpha, \mathfrak{F})$ are given respectively in terms of morphisms in $\mathcal{K} l(T)$ by: $\|\alpha, \mathfrak{F}\|: X \multimap 1$ and $\|\alpha, \mathfrak{F}\|_{\omega}: X \multimap 0$, where

$$
\|\alpha, \mathfrak{F}\| \triangleq ! \cdot \mathfrak{f}_{\mathfrak{F}} \cdot \alpha^{*} \text { and }\|\alpha, \mathfrak{F}\|_{\omega} \triangleq\left(\mathfrak{f}_{\mathfrak{F}} \cdot \alpha^{+}\right)^{\omega} \text {. }
$$

Finite behaviour of a state $x \in X$ of $(\alpha, \mathfrak{F})$ is the map $\|\alpha, \mathfrak{F}\| \cdot x_{X}: 1 \multimap \rightarrow 1$, and its $\omega$-behaviour is given by $\|\alpha, \mathfrak{F}\|_{\omega} \cdot x_{X}: 1 \multimap 0$. Here,

$$
x_{X}: 1 \multimap X=1 \rightarrow T X ; 1 \mapsto \eta_{X}(x) .
$$

Example 5.6. As we have already seen in Section 3, the finite and $\omega$-behaviour of $\mathcal{P}\left(\Sigma^{*} \times\right.$ $\mathcal{I} d+\Sigma^{\omega}$ )-automata coincides with the classical notions whenever the tuple is given by $(\alpha: n \rightarrow \mathcal{P}(\Sigma \times n), \mathfrak{F} \subseteq n)$. The same applies to tree automata (see Proposition 3.2).

5.2. Additional remarks. Our approach to defining semantics for coalgebras seems to diverge slightly from the established coalgebraic takes known from e.g. [HJS07, SW13, BMSZ15, USH16]. The purpose of this subsection is to compare our setting with the frameworks presented in the literature and try to justify the (slight) differences.

Our approach builds on top of two fixpoint operators, namely $(-)^{*}$ and $(-)^{\omega}$. The choice of these two operators, and not other (e.g. the dagger operator from [ÉK11, BMSZ15]) follows from the premise that we wanted to make the connection with the classical results in regular and $\omega$-regular languages as clear and as direct as possible. As we witness here, the classical Kleene star operation and $(-)^{\omega}$ [PP04] prove to have their general categorical counterparts.

It may not be clear to the reader why the finite and $\omega$-behaviour maps have different codomains, i.e. the former is a map $X \multimap \rightarrow 1$ and the latter $X \multimap \rightarrow 0$. Let us focus on the finite behaviour first. So far in the coalgebraic literature, finite behaviour of systems was introduced in terms of finite trace [SW13, BMSZ15, JSS12]. In the setting of systems $X \rightarrow T F X$ it is obtained in terms of the initial algebra-final coalgebra coincidence [HJS07, BMSZ15]. When translated to the setting of systems with internal moves, the finite trace is given by $\mu x . x \cdot \alpha: X \multimap \rightarrow 0$ and is calculated in the Kleisli category for the monad $T F^{*}$ [Bre14, Bre15]. However, this holds for coalgebras whose type monad encodes accepting states. From the point of our setting, the accepting states are not part of the transition and are encoded in terms of $\mathfrak{F} \subseteq X$ instead. The direct use of initial algebra-final coalgebra coincidence makes no sense here, as the initial algebra would simply be degenerate. Luckily, there is a simple formal argument showing that our approach from this paper and the aforementioned approach established in the coalgebraic literature coincide. For the monad $T$ and a $T$-automaton $(\alpha, \mathfrak{F})$ consider the monad $T(\mathcal{I} d+1)^{9}$ and the map $X \rightarrow T(X+1)$ defined for any $x \in X$ by $\alpha(x) \vee \chi_{\mathfrak{F}}(x)$, where $\chi_{\mathfrak{F}}: X \rightarrow T(X+1) ; x \mapsto\left\{\begin{array}{cc}\eta_{X+1}(1) & \text { if } x \in \mathfrak{F}, \\ \perp & \text { otherwise. }\end{array}\right.$ It is a simple exercise to prove that the least fixpoint $\mu x \cdot x \cdot\left(\alpha \vee \chi_{\mathfrak{F}}\right): X \multimap \rightarrow 0=X \rightarrow T(0+1)=X \rightarrow T 1$ calculated in $\mathcal{K} l(T(\mathcal{I} d+1))$ is the same as the finite behaviour map $\|\alpha, \mathfrak{F}\|: X \rightarrow 1=X \rightarrow T 1$ calculated in $\mathcal{K l}(T)$. Therefore, our definition of finite behaviour via $(-)^{*}$ coincides with the coalgebraic finite trace semantics via $\mu x . x \cdot \alpha$.

The finite behaviour of a state of an automaton from Definition 5.5 is of type $1 \multimap \rightarrow 1$. We argue that this map should be viewed as a generalization of a finitary language. Classically,

\footnotetext{
${ }^{9}$ It can be easily verified that for any monad $T$ the functor $T(\mathcal{I} d+1)$ carries a monadic structure. It follows from the fact that the exception monad $\mathcal{I} d+E$ induces an exception monad transfomer $T \mapsto T(\mathcal{I} d+E)$.
} 
these languages have been considered in some algebraic context, e.g. with the familiar algebraic operations of concatenation, Kleene start closure and finite union. These operations considered on our abstract categorical level directly translate into morphism composition, saturation and finite joins of endomorphisms $1 \multimap \rightarrow 1$ respectively.

As far as the infinite behaviour is concerned, it should be noted here that our prototypical example of a monad is $T F^{\infty}=T\left(F^{*} \oplus F^{\omega}\right)$ from Section 4. By Theorem 4.3 the object $\left(F^{*} \oplus F^{\omega}\right)(0)=F^{\omega}$ is the carrier of the terminal $F$-coalgebra $\zeta: F^{\omega} \rightarrow F F^{\omega}$ making $T F^{\infty} 0=T F^{\omega}$. This is exactly what we expect to have as a codomain of an infinite trace map (see also for comparison [USH16, Cîr10]).

Additionally, the type of the infinite behaviour of a state of an automaton is $1 \multimap \rightarrow 0$ and it reflects the partial algebraic nature of (in)finitary languages. In particular, it makes sense to compose (concatenate) a finitary langugage $1 \rightarrow 1$ with an infinitary language $1 \rightarrow 0$ and get an infinitary one $(1 \multimap 1 \rightarrow 0=1 \multimap \rightarrow 0)$, but not vice versa. Moreover, it does not necessarily make sense to compose two infinitary languages $(1 \multimap 0$ and $1 \multimap \rightarrow 0)$ with each other.

5.3. Kleene theorems. The purpose of this part of the paper is to state and prove Kleene theorems akin to Proposition 3.4 and 3.5. These theorems require us to work with finite automata and their behaviour, so we will restrict the setting of this subsection to the Lawvere theory $\mathbb{T}$ associated with the monad $T$.

In this subsection we consider a set $\mathcal{A}$ of endomorphisms from $\mathbb{T}$ such that:

- $\mathcal{A}$ contains all base map endomorphisms,

- $\perp_{n, n}: n \rightarrow n \in \mathcal{A}$ for any $n<\omega^{10}$,

- if $\left\{\alpha_{k}: n_{i} \multimap \rightarrow n_{i}\right\}_{k=1, \ldots, k} \subseteq \mathcal{A}$ then $\alpha_{1}+\ldots+\alpha_{k} \in \mathcal{A}$,

- $\mathcal{A}$ is closed under taking finite suprema.

The set $\mathcal{A}$ plays a role of a set of admissible transition functions for automata taken into consideration. A $T$-automaton $(\alpha, \mathfrak{F})$ whose transition $\alpha: n \rightarrow \rightarrow n$ is an arrow in $\mathbb{T}$ is called $\mathcal{A}$-automaton if $\alpha \in \mathcal{A}$.

Example 5.7. In the case of our leading examples of theories, namely LTS ${ }^{\omega}$ and TTS $^{\omega}$, the prototypical choice for $\mathcal{A}$ was given in condition (a) in Proposition 3.5 and 3.4 respectively.

Definition 5.8. The set of regular morphisms $m \rightarrow p \in \mathbb{T}$ is defined by:

$$
\begin{aligned}
\mathfrak{R e g}(m, p) \triangleq & \left\{j^{\prime} \cdot \mathfrak{f}_{\mathfrak{F}} \cdot \alpha^{*} \cdot j \mid(\alpha: n \multimap \rightarrow n, \mathfrak{F}) \text { is a } \mathcal{A}\right. \text {-automaton and } \\
& \left.j: m \multimap n, j^{\prime}: n \multimap p \text { are base maps }\right\} .
\end{aligned}
$$

The set of regular morphisms $\mathfrak{R e g}(1, p)$ will be often referred to as the set of regular trees with variables in $p$. Note that $\mathfrak{R e g}(1,1)$ is exactly the set of finite behaviours of states in $\mathcal{A}$-automata.

We list the statements without proofs which we later provide in Subsection 5.3.1.

Lemma 5.9. The identity maps in $\mathbb{T}$ are regular morphisms. Moreover, regular morphisms are closed under composition from $\mathbb{T}$.

\footnotetext{
${ }^{10}$ In order to simplify the notation we will often omit the subscript and write $\perp$ to denote $\perp_{n, m}$ if the domain and codomain of $\perp$ can be deduced from the context.
} 
The above lemma precisely says that the collection of objects $n=0,1, \ldots$ with morphisms $\mathfrak{R e g}(m, n)$ forms a category with the composition taken from $\mathbb{T}$. We denote this category by $\mathfrak{R e g}(\mathcal{A})$. We have the following.

Theorem 5.10 (Kleene theorem for regular behaviour). The category $\mathfrak{R e g}(\mathcal{A})$ is a subtheory of $\mathbb{T}$ such that:

(a) it contains all maps from $\mathcal{A}$,

(b) it admits finite suprema,

(c) its endomorphisms are closed under $(-)^{*}$.

Moreover, if $\mathfrak{R a t}(\mathcal{A})$ denotes the smallest subtheory of $\mathbb{T}$ which satsfies (a)-(c) then

$$
\mathfrak{R a t}(\mathcal{A})=\mathfrak{R e g}(\mathcal{A}) .
$$

Finally, we define

$$
\begin{aligned}
& \omega \mathfrak{R a t}(\mathcal{A}) \triangleq\left\{\left[r_{1}, \ldots, r_{m}\right]^{\omega} \cdot r \mid r, r_{i} \in \mathfrak{R a t}(1, m) \text { for } m<\omega\right\} \\
& \omega \mathfrak{R e g}(\mathcal{A}) \triangleq\left\{\|\alpha, \mathfrak{F}\|_{\omega} \cdot i_{n}: 1 \multimap \rightarrow 0 \mid(\alpha, \mathfrak{F}) \text { is an } \mathcal{A} \text {-automaton }\right\} .
\end{aligned}
$$

Theorem 5.11 (Kleene theorem for $\omega$-regular behaviour). We have

$$
\omega \mathfrak{R a t}(\mathcal{A})=\omega \mathfrak{R e g}(\mathcal{A}) .
$$

5.3.1. Proofs. The purpose of this subsection is to present the proofs of the statements above. Before we proceed we would like to make a remark concerning the material presented here and its originality. Several Kleene theorems (akin to Theorem 5.10) have been presented and proven in the literature on the level of iteration theories (see e.g. [Ési97, BE93, EH09, ÉK11, ÉK13]). According to our knowledge, due to minor differences in the formulation, the theorems presented in our paper do not fit directly into any existing setting. However, the classical proof techniques used in loc. cit. are still applicable here. These methods are based on using well know properties satisfied by a fixpoint operator. We recall them here and present detailed proofs of our statements. We additionally use string diagrams as a visual aid to help the reader understand the techniques better.

In order to proceed with the proofs we need to introduce some new notions and define a notation used below. We start off by defining $[\mathcal{A}]$ to be the set of morphisms from $\mathbb{T}$ obtained by (pre- and post-)composing maps from $\mathcal{A}$ with base morphisms with suitable domains and codomains:

$$
[\mathcal{A}] \triangleq\{i \cdot \alpha \cdot j \mid \alpha: n \multimap n \in \mathcal{A} \text { and } i: n \multimap p, j: m \multimap n \text { are base maps }\} .
$$

Moreover, since the proofs presented below use the identity GSPI which requires an extended definition of the saturation operator, for any morphism $\alpha: n \rightarrow n+p$ we define:

$$
\alpha^{\otimes} \triangleq\left[\alpha, \mathrm{in}_{n+p}^{p}\right]^{*} \cdot \mathrm{in}_{n+p}^{n} .
$$

Note that $\left[\alpha^{\otimes}, \operatorname{in}_{n+p}^{p}\right]=\left[\alpha, \operatorname{in}_{n+p}^{p}\right]^{*}$. Hence, if $p=0$ then $\alpha^{\otimes}=\alpha^{*}$ for $\alpha: n \multimap \rightarrow n+p$. 
String diagram notation. Let us now develop a string diagram notation which will clarify the proofs considerably.

Remark 5.12. It is important to emphasize that the purpose of the new notation is to build a visual aid to the technical statements made below. The reader should note that all proofs presented here are written so that the knowledge of the string diagram calculus is not required. However, given the complexity of some of the (in)equalities used, we strongly believe that the diagrammatic notation improves their readability (conf. e.g. GSPI and its diagrammatic representation). Hence, we decide to proceed with its introduction.

We adopt the standard string diagram calculus for monoidal categories [Sel11, FS18] which will be tailored to our purposes. A morphism $f: m \rightarrow n$ is depicted by $\sqrt[m]{f}$. We will often drop the (co)domain types from the notation and depict $f$ simply by $-f$. If $f: m \rightarrow n+p$ and the coproduct codomain needs to be emphasized by the diagram notation then we depict $f$ by $-f=$. This generalizes to $m_{1}+\cdots+m_{k} \rightarrow \rightarrow n_{1}+\cdots+n_{l}$ in an obvious manner. Whenever $f: m \rightarrow n$ and $g: n \rightarrow \rightarrow p$ then the composition $g \cdot f: m \rightarrow \rightarrow p$ is $f f$. Given two maps $f: m_{1} \multimap n_{1}$ and $g: m_{2} \multimap \rightarrow n_{2}$ the coproduct $f+g$ : $m_{1}+m_{2} \multimap \rightarrow n_{1}+n_{2}$ is depicted by

$$
\begin{aligned}
& -f \\
& -g-
\end{aligned}
$$

Given any endomorphism $\alpha: n \multimap \rightarrow n$ we depict the saturated map $\alpha^{*}$ by

$$
\alpha-\text {. }
$$

By slightly abusing the notation we extend it to the generalized saturation $(-)^{\otimes}$ operator and for any $\alpha: n \multimap n+p$ denote $\alpha^{\otimes}: n \multimap \rightarrow n+p$ diagrammatically by:

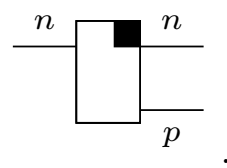

We will use a separate notation to denote special morphisms. The identity map id $: n \rightarrow \rightarrow n$ is depicted by - , the maps $\perp_{0, m}: 0 \multimap \rightarrow m$ and $\perp_{m, 0}: m \multimap \rightarrow 0$ by $\circ$ and $\longrightarrow$ respectively.

Since by Assumption (C) we have $\perp_{m, n}=\perp_{0, n} \cdot \perp_{m, 0}$ we depict $\perp_{m, n}$ by

$$
\longrightarrow \circ \text {. }
$$

Additionally, since the map in $\mathrm{in}_{m+n}^{m}: m \rightarrow \rightarrow m+n$ satisfies $\mathrm{in}_{m+n}^{m}=\mathrm{id}_{m}+\perp_{0, n}$, its diagrammatic representation is

$$
\overline{\mathrm{O}} \text {. }
$$

The cotuple string diagram notation has already been presented in Subsection 2.7. However, since the the cotuple

$$
\left[\perp_{m, n}: m \multimap \rightarrow n, f: n \multimap \rightarrow n\right]: m+n \multimap \rightarrow n
$$

satisfies

$$
\left[\perp_{m, n}: m \multimap \rightarrow n, f: n \multimap \rightarrow n\right]=\perp_{m, 0}+f
$$

the following diagram depicts it: 


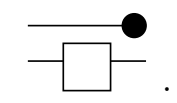

Moreover, the morphism $\left[\alpha, \mathrm{in}_{n+p}^{p}\right]: n+p \multimap \rightarrow n+p$ satisfies the identity

$$
\left[\alpha, \mathrm{in}_{n+p}^{p}\right]=\left(\mathrm{id}_{n}+\left[\mathrm{id}_{p}, \mathrm{id}_{p}\right]\right) \cdot\left(\alpha+\mathrm{id}_{p}\right)
$$

and its diagrammatic representation is

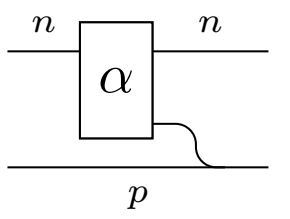

We are now ready to list some basic observations and remarks about the diagram calculus introduced above. First of all note that by the properties of saturation the following diagram (in)equalities hold:

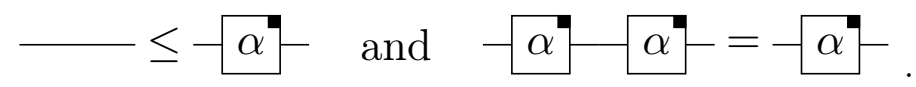

Moreover, since $\perp$ satisfies $f \cdot \perp=\perp$ (Assumption (C)) for any $f$, we have:

$$
f \cdot[\perp, \mathrm{id}]=[f \cdot \perp, f \cdot \mathrm{id}]=[\perp, f],
$$

which diagrammatically is represented in terms of the following identity:

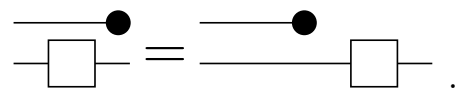

In the above, the right hand side of the equality, namely $\stackrel{\bullet}{\square-}$, is the composition of $\longrightarrow$ and $-\square$.

Generalized star pairing identity. Here, we present the so-called generalized star pairing identity described in any Lawvere theory equipped with an operator $(-)^{\otimes}$ assigning to each morphism $f: n \multimap n+p$ a morphism $f^{\otimes}: n \multimap \rightarrow n+p$ [EH09]. This identity will hold in our setting and will be used in the proof of Theorem 5.10 and lemmas that precede it.

For $f: n \multimap \rightarrow n+m+p$ and $g: m \multimap \rightarrow n+m+p$ the generalized star pairing identity is [EH09]:

$$
[f, g]^{\otimes}=\left[\left[\mathrm{in}_{n+m+p}^{n},\left(\pi^{-1}+\mathrm{id}_{p}\right) \cdot k^{\otimes}, \mathrm{in}_{n+m+p}^{p}\right] \cdot f^{\otimes},\left(\pi^{-1}+\mathrm{id}_{p}\right) \cdot k^{\otimes}\right],
$$

where $\pi: m+n \multimap n+m$ is given by $\pi \triangleq\left[\mathrm{in}_{n+m}^{m}, \mathrm{in}_{n+m}^{n}\right]$ and

$$
k \triangleq\left[\left(\pi+\mathrm{id}_{p}\right) \cdot f^{\otimes},\left[\mathrm{in}_{m+n+p}^{m}, \mathrm{in}_{m+n+p}^{p}\right]\right] \cdot g .
$$

The generalized star pairing identity is depicted by the string diagram: 

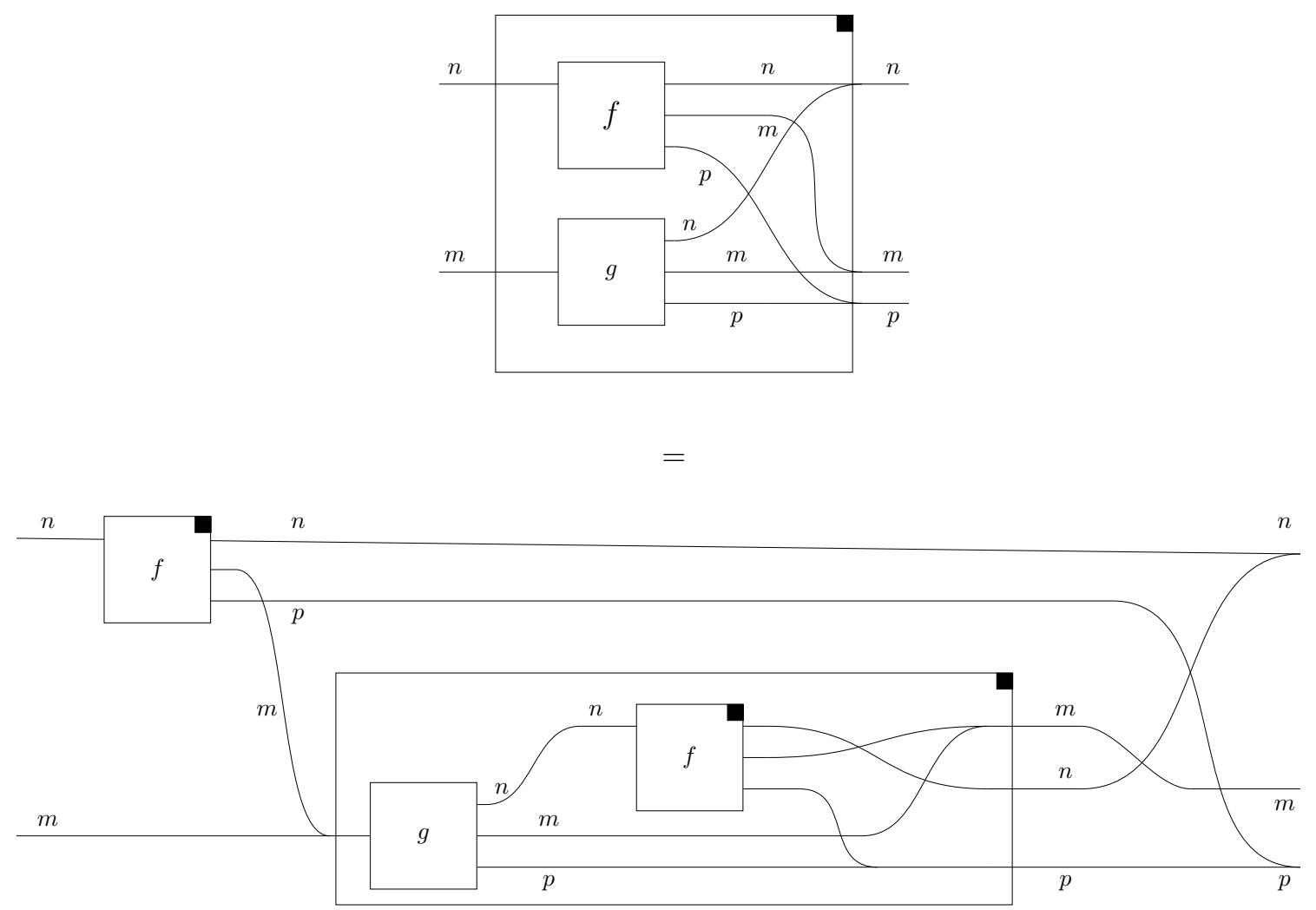

Rational morphisms. Let $\mathfrak{R a t}(A)$ be the theory defined in Theorem 5.10. Since $(-)^{*}$ (with its extension $(-)^{\otimes}$ given in 5.1) is defined in $\mathfrak{R a t}(\mathcal{A})$ in terms of a least fixpoint operator in a more general setting of the Kleisli category for $T$ which satisfies Assumption 5.2, we have the following [Ési97, EH09] ${ }^{11}$ (see also [BE93, ÉK11, ÉK13]):

Lemma 5.13. The theory $\mathfrak{R a t}(A)$ satisfies the generalized star pairing identity for the operator $(-)^{\otimes}$.

Regular morphisms and normal form. Note that any regular map is a morphism in $\mathfrak{R a t}(A)$. In particular, this means that regular morphisms satisfy the generalized star pairing identity.

Let us first introduce a new notion.

Definition 5.14. A morphism $r: m \rightarrow \rightarrow p$ is said to be in normal form if

$$
r=\left[\perp_{n, p}, \mathrm{id}_{p}\right] \cdot \alpha^{\otimes} \cdot \mathrm{in}_{n}^{m}
$$

for some $\alpha: n \multimap \rightarrow n+p \in[\mathcal{A}]$ and $m \leq n$.

\footnotetext{
${ }^{11}$ Here, we sketch a proof of Lemma 5.13. The generalized star pairing identity is equivalent to the so-called pairing identity given in dagger theories which are also grove theories, where the dagger operator is compatible with the star operator [EH09]. The pairing identity for dagger theories holds in any $\omega$-continuous theory [Ési97]. Our theory $\mathfrak{R a t}(\mathcal{A})$ satisfies the assumptions of an $\omega$-continuous grove theory where the dagger operator is given by $(\alpha: n \rightarrow n+p) \mapsto(\mu x \cdot x \cdot \alpha: n \rightarrow-p)$ and the extended saturation operator 5.1 is compatible with it. This completes the proof. We skip the definitions of the theories and new notions introduced in the footnote and refer the reader to loc. cit. for details.
} 
Let the family of all maps in normal form be denoted by $N F(\mathcal{A})$. The map $\alpha$ and $r$ in $N F(\mathcal{A})$ from Definition 5.14 are depicted by the string diagrams below:
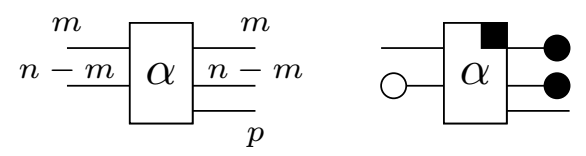

The right-hand-side diagram is a correct representation of $r$ as it is the result of the composition of three maps:

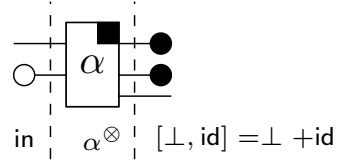

It follows straight by Definition 5.14 that every map in normal form is a regular morphism. Note that the family of maps in normal form contains all base maps and all morphisms from $[\mathcal{A}]$. Additionally, it is closed under cotupling $[-,-]$. Moreover, the following statement holds.

Lemma 5.15. The family $N F(\mathcal{A})$ is closed under the composition $\cdot$, finite suprema $\vee$ and saturation $(-)^{*}$.

Proof. The proof is divided into three parts.

Part 1. Here, we show that the family $N F(\mathcal{A})$ is closed under the composition. Take $r_{1}=\left[\perp_{n_{1}, m_{2}}, \mathrm{id}_{m_{2}}\right] \cdot \alpha^{\otimes} \cdot \mathrm{in}_{n_{1}}^{m_{1}}$ for $m_{1} \leq n_{1}$ and $r_{2}=\left[\perp_{n_{2}, m_{3}}, \mathrm{id}_{m_{3}}\right] \cdot \beta^{\otimes} \cdot \mathrm{in}_{n_{2}}^{m_{2}}$ for $m_{2} \leq n_{2}$, where $\alpha: n_{1} \multimap n_{1}+m_{2}$ and $\beta: n_{2} \multimap n_{2}+m_{3}$. Consider morphisms $f: n_{1} \multimap n_{1}+n_{2}+m_{3}$ and $g: n_{2} \multimap \rightarrow n_{1}+n_{2}+m_{3}$ defined by

$$
f \triangleq \alpha+\perp_{0, n_{2}-m_{2}}+\perp_{0, m_{3}} \text { and } g \triangleq \perp_{0, n_{1}}+\beta
$$

and represented in terms of their string diagrams respectively as follows:
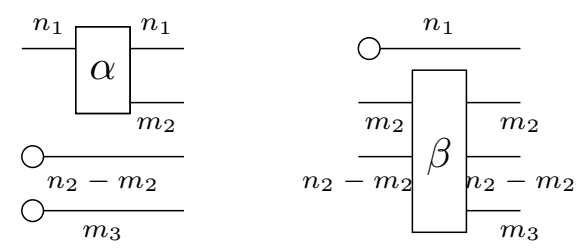

Let $\gamma=[f, g]$. Then $\gamma: n_{1}+n_{2} \multimap \rightarrow n_{1}+n_{2}+m_{3}$ is in $[\mathcal{A}]$ and by a careful analysis of the generalized star pairing identity it follows that the morphism $\left(\pi^{-1}+\mathrm{id}\right) \cdot k^{\otimes}$ in GSPI is, in our case, given by $\perp_{0, n_{1}}+\beta^{\otimes}$ which is diagrammatically captured by:

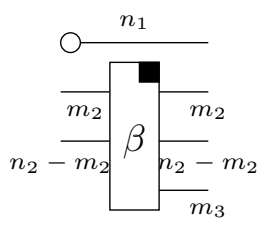

Hence, by GSPI and 5.2 we get:

$$
r_{2} \cdot r_{1}=\left[\perp, \mathrm{id}_{m_{3}}\right] \cdot \gamma^{\otimes} \cdot \mathrm{in}^{m_{1}} .
$$


Part 2. Here, we show that given two maps $r_{1}=\left[\perp_{n_{1}, m_{2}}, \mathrm{id}_{m_{2}}\right] \cdot \alpha^{\otimes} \cdot \mathrm{in}_{n_{1}+m_{2}}^{m_{1}}$ and $r_{2}=$ $\left[\perp_{n_{2}, m_{2}}, \mathrm{id}_{m_{2}}\right] \cdot \beta^{\otimes} \cdot \mathrm{in}_{n_{2}+m_{2}}^{m_{1}}$ for $\alpha: n_{1} \multimap \rightarrow n_{1}+m_{2}$ and $\beta: n_{2} \multimap \rightarrow n_{2}+m_{2}$ their join $r_{1} \vee r_{2}$ is in normal form. Let $f: m_{1} \rightarrow m_{1}+\left(n_{1}+n_{2}\right)+m_{3}$ be defined in terms of supremum of the following two morphisms:

$$
\begin{aligned}
& \left(\perp_{0, m_{1}}+\mathrm{id}_{m_{1}+\left(n_{1}-m_{1}\right)+n_{2}+m_{2}}\right) \cdot\left(\mathrm{id}_{m_{1}}+\perp_{0,\left(n_{1}-m_{1}\right)+n_{2}+m_{2}}\right)= \\
& \left(\perp_{0, m_{1}}+\mathrm{id}_{m_{1}}+\mathrm{id}_{n_{1}-m_{1}}+\mathrm{id}_{n_{2}}+\mathrm{id}_{m_{2}}\right) \cdot\left(\mathrm{id}_{m_{1}}+\perp_{0, n_{1}-m_{1}}+\perp_{0, n_{2}}+\perp_{0, m_{2}}\right)
\end{aligned}
$$

and

$$
\begin{aligned}
& \left(\perp_{0, m_{1}}+\mathrm{id}_{m_{1}+\left(n_{1}-m_{1}\right)+n_{2}+m_{2}}\right) \cdot \sigma \cdot\left(\mathrm{id}_{m_{1}}+\perp_{0,\left(n_{1}-m_{1}\right)+n_{2}+m_{2}}\right)= \\
& \left(\perp_{0, m_{1}}+\mathrm{id}_{m_{1}}+\mathrm{id}_{n_{1}-m_{1}}+\mathrm{id}_{n_{2}}+\mathrm{id}_{m_{2}}\right) \cdot \sigma \cdot\left(\mathrm{id}_{m_{1}}+\perp_{0, n_{1}-m_{1}}+\perp_{0, n_{2}}+\perp_{0, m_{2}}\right),
\end{aligned}
$$

where the endomorphism

$$
\sigma: m_{1}+\left(n_{1}-m_{1}\right)+m_{1}+\left(n_{2}-m_{1}\right)+m_{2} \rightarrow m_{1}+\left(n_{1}-m_{1}\right)+m_{1}+\left(n_{2}-m_{1}\right)+m_{2}
$$

permutes the first and third component of the coproduct and is the identity everywhere else. Now, let $g: n_{1}+n_{2} \rightarrow m_{1}+\left(n_{1}+n_{2}\right)+m_{2}$ be given by:

$$
g \triangleq \sigma^{\prime} \cdot\left(\perp_{0, m_{1}}+\alpha+\beta\right)
$$

where the endomorphism $\sigma^{\prime}: m_{1}+n_{1}+m_{2}+n_{2}+m_{2} \rightarrow \rightarrow m_{1}+n_{1}+m_{2}+n_{2}+m_{2}$ injects the third component of the coproduct into the last component and is the identity everywhere else. The morphisms $f$ and $g$ are depicted in terms of their string diagrams respectively as follows:
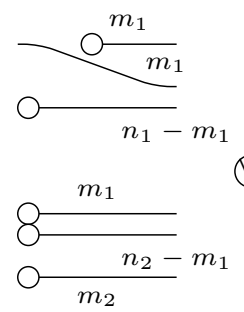

(1)

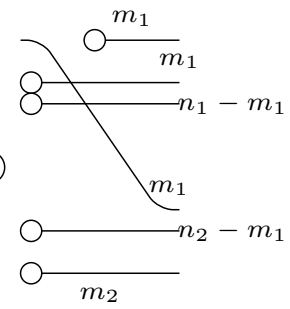

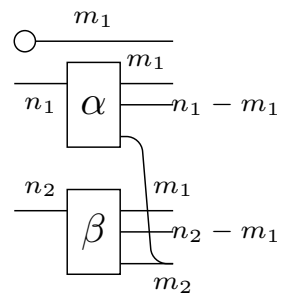

Let $\gamma=[f, g]$. Then $\gamma: m_{1}+\left(n_{1}+n_{2}\right) \multimap m_{1}+\left(n_{1}+n_{2}\right)+m_{2}$ is in $[\mathcal{A}]$. The morphism $f^{\otimes}$ is equal to $f \vee\left(\mathrm{id}_{m_{1}}+\perp_{0, n_{1}+n_{2}+m_{2}}\right)$ and is depicted by:

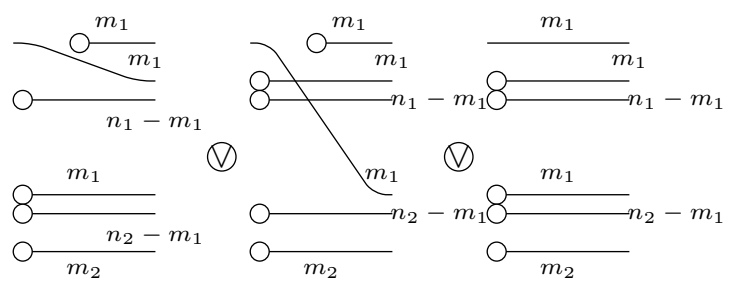

Moreover, $\left(\pi^{-1}+\right.$ id $) \cdot k^{\otimes}$ from GSPI is, in our case, given by $\sigma^{\prime} \cdot\left(\perp_{0, m_{1}}+\alpha^{\otimes}+\beta^{\otimes}\right)$, where $\sigma^{\prime}$ is as above and is depiced as follows: 


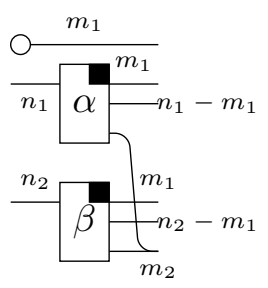

As before, by a careful analysis of GSPI and 5.2 we get:

$$
r_{1} \vee r_{2}=\left[\perp, \mathrm{id}_{m_{2}}\right] \cdot \gamma^{\otimes} \cdot \mathrm{in}^{m_{1}} .
$$

Part 3. Finally, we show that for a map $r: m \rightarrow \rightarrow$ given by its normal form $r=[\perp$ , $\left.\mathrm{id}_{m}\right] \cdot \alpha^{\otimes} \cdot \mathrm{in}_{n}^{m}$ for $\alpha: n \rightarrow n+m$ its saturation $r^{*}$ is in $N F(\mathcal{A})$. The proof presented here uses a construction of a morphism that will later be used in the proof of Theorem 5.11 and the lemmas that precede it.

Let $\alpha^{\prime}: n+m \rightarrow n+m$ be given by $\alpha^{\prime}=\left[\alpha, \mathrm{in}_{n+m}^{m}\right]$ and consider $\gamma: n+m \rightarrow n+m$ defined by

$$
\gamma \triangleq \alpha^{\prime} \vee \sigma
$$

where $\sigma: m+(n-m)+m \rightarrow \rightarrow m+(n-m)+m$ injects the third component of the coproduct into the first one and is the identity everywhere else. The map $\gamma$ is depicted by the following diagram:

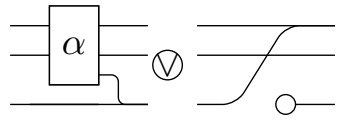

Note that $\gamma$ is a $[\mathcal{A}]$-map which satisfies $\gamma^{*}=\gamma^{*}$ for $\gamma^{\prime}=\alpha^{\prime *} \vee \sigma$ represented by the diagram:

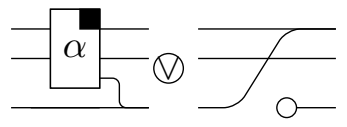

We have $\left[\perp, \mathrm{id}_{m}\right] \cdot \gamma^{\prime}=\left[\perp, \mathrm{id}_{m}\right] \cdot \alpha^{\prime *}$ which is depicted by:

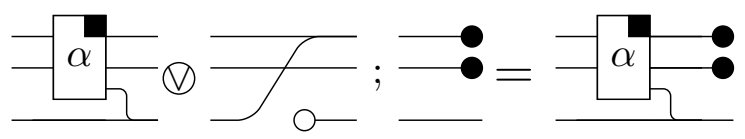

Moreover, $\left[\perp, \mathrm{id}_{m}\right] \cdot \gamma^{\prime 2}=$

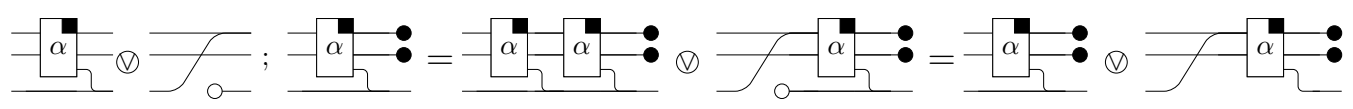

Hence, by right distributivity w.r.t. the base morphisms we have $\left[\perp, \mathrm{id}_{m}\right] \cdot \gamma^{\prime 2} \cdot \mathrm{in}_{n+m}^{m}=\mathrm{id}_{m} \vee r$ and $\left[\perp, \mathrm{id}_{m}\right] \cdot \gamma^{\prime 2} \cdot \mathrm{in}^{n}=\left[\perp, \mathrm{id}_{m}\right] \cdot \gamma^{\prime} \cdot \mathrm{in}^{n}$. Since $\left[\perp, \mathrm{id}_{m}\right] \cdot \gamma^{\prime} \cdot \mathrm{in}_{n+m}^{m}=\mathrm{id}_{m}$ we conclude that:

$$
\left[\perp, \mathrm{id}_{m}\right] \cdot \gamma^{\prime 2} \leq(\mathrm{id} \vee r) \cdot\left[\perp, \mathrm{id}_{m}\right] \cdot \gamma^{\prime} .
$$

Moreover,

$$
\left[\perp, \mathrm{id}_{m}\right] \cdot \gamma^{\prime 2}=\left[r^{\prime}, \mathrm{id} \vee r\right] \geq\left[\perp, \mathrm{id}_{m} \vee r\right]=(\mathrm{id} \vee r) \cdot\left[\perp, \mathrm{id}_{m}\right],
$$

for $r^{\prime} \triangleq[\perp, \mathrm{id}] \cdot \alpha^{*}$. Note that $r^{\prime} \cdot \mathrm{in}_{n}^{m}=r$. Hence, to summarize:

$$
(\text { id } \vee r) \cdot[\perp, \text { id }]=[\perp, \text { id } \vee r] \leq\left[r^{\prime}, \text { id } \vee r\right] \leq[\perp, \text { id }] \cdot \gamma^{\prime 2} \leq(\text { id } \vee r) \cdot[\perp, \text { id }] \cdot \gamma^{\prime} .
$$


If we let $f=[\perp$, id $]$ and $g=f \cdot \gamma^{\prime}$ then the above inequalities are rephrased as follows:

$$
\text { (id } \vee r) \cdot f \leq f \cdot\left(\gamma^{\prime}\right)^{2} \text { and } g \cdot \gamma^{\prime} \leq(\text { id } \vee r) \cdot g \text {. }
$$

By 1 in Lemma 5.4, (B) in Assumption 5.2 and id $\leq \gamma^{\prime}$ we get:

$$
r^{*} \cdot f \leq f \cdot \gamma^{* *}=g \cdot \gamma^{*} \leq r^{*} \cdot g .
$$

Since $f \cdot \operatorname{in}_{n+m}^{m}=g \cdot \mathrm{in}_{n+m}^{m}=\mathrm{id}_{m}$ and $\gamma^{*}=\gamma^{*}$ we get: $r^{*}=[\perp$, id $] \cdot \gamma^{*} \cdot \mathrm{in}_{n+m}^{m}$. Similarily, we show $r^{+}=[\perp, \mathrm{id}] \cdot \gamma^{*} \cdot \mathrm{in}_{n+m}^{n} \cdot \mathrm{in}_{n}^{m}$. This proves that $r^{+}, r^{*} \in N F(\mathcal{A})$.

We are now ready to present the following proof.

Proof. (Theorem 5.10) Note that all maps from $N F(\mathcal{A})$ are regular and all regular maps are in $\mathfrak{R a t}(\mathcal{A})$. By Lemma 5.15 morphisms from $N F(\mathcal{A})$ form a theory closed under finite suprema and saturation. Hence, $N F(\mathcal{A})=\mathfrak{R e g}(\mathcal{A})=\mathfrak{R a t}(\mathcal{A})$. This completes the proof.

Before we proceed with the proof of Theorem 5.11 we require one extra statement. Let us define:

$$
\begin{aligned}
& \omega \mathfrak{R a t}(\mathcal{A})(n) \triangleq\left\{\left[r_{1}, \ldots, r_{m}\right]^{\omega} \cdot r \mid r \in \mathfrak{R e g}(n, m), r_{i} \in \mathfrak{R e g}(1, m) \text { for } m<\omega\right\}, \\
& \omega \mathfrak{R e g}(\mathcal{A})(n) \triangleq\left\{\|\alpha, \mathfrak{F}\|_{\omega} \cdot \operatorname{in}_{m}^{n}: n \multimap 0 \mid(\alpha, \mathfrak{F}) \text { is } \mathcal{A} \text {-aut. with } \alpha: m \rightarrow \rightarrow m\right\} .
\end{aligned}
$$

and note that $\omega \mathfrak{R a t}(\mathcal{A})=\omega \mathfrak{R a t}(\mathcal{A})(1)$ and $\omega \mathfrak{R e g}(\mathcal{A})=\omega \mathfrak{R e g}(\mathcal{A})(1)$. Additionally, the following holds.

Lemma 5.16. For any $r \in \mathfrak{R e g}(m, m)$ we have:

$$
r^{\omega} \in \omega \mathfrak{R e g}(\mathcal{A})(m) .
$$

Proof. All regular maps can be given in their normal form. Hence, we have

$$
r=\left[\perp, \mathrm{id}_{m}\right] \cdot \alpha^{\otimes} \cdot \mathrm{in}_{n}^{m}
$$

for $\alpha: n \rightarrow n+m$, where $m \leq n$. We depict $r$ by $\gamma \triangleq[\alpha$, in $] \vee \sigma=\alpha^{\prime} \vee \sigma$ defined as in 5.3 depicted by the following diagram:

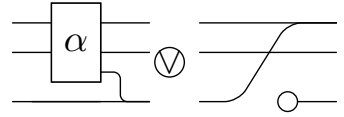

By the properties listed in Part 3. of the proof of Lemma 5.15 we have:

$$
r^{+}=[\perp, \mathrm{id}] \cdot \gamma^{*} \cdot \mathrm{in}_{n+m}^{n} \cdot \mathrm{in}_{n}^{m} .
$$

Moreover, let $\xi$ be defined by $\xi \triangleq=\sigma \cdot \alpha^{\prime}=\sigma \cdot[\alpha$, in $]$ and depicted in the following diagram:

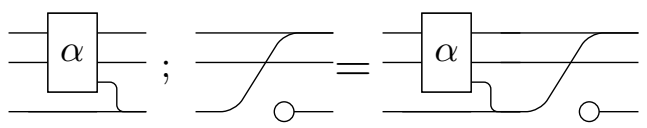

The map $\xi$ is a $[\mathcal{A}]$-map which satisfies $\xi^{*}=\gamma^{*}$. Additionally,

$$
\begin{aligned}
& r^{+}=[\perp, \mathrm{id}] \cdot \xi^{*} \cdot \mathrm{in}_{n+m}^{n} \cdot \operatorname{in}_{n}^{m}= \\
& {\left[\perp, \mathrm{id}_{m}\right] \cdot \xi^{*} \cdot \xi \cdot \mathrm{in}_{n+m}^{m}=} \\
& {\left[\perp, \mathrm{id}_{m}\right] \cdot \xi^{+} \cdot \operatorname{in}_{n+m}^{m} .}
\end{aligned}
$$


Since $(\perp+\mathrm{id})=\mathrm{in}_{n+m}^{m} \cdot[\perp$, id $]$ we get that

$$
\mathrm{in}_{n+m}^{m} \cdot r^{+}=\mathrm{in}^{m} \cdot\left[\perp, \mathrm{id}_{m}\right] \cdot \xi^{+} \cdot \mathrm{in}_{n+m}^{m}=\left(\perp+\mathrm{id}_{m}\right) \cdot \xi^{+} \cdot \mathrm{in}_{n+m}^{m} .
$$

From the above we get:

$$
r^{\omega} \triangleq\left(r^{+}\right)^{\omega} \stackrel{\dagger}{=}\left(\left(\perp+\mathrm{id}_{m}\right) \cdot \xi^{+}\right)^{\omega} \cdot \mathrm{in}_{n+m}^{m} .
$$

The identity $(\diamond)$ follows by Lemma 5.4. The identity $(\dagger)$ follows from a more general property: given two coalgebras $\alpha: X \multimap X=X \rightarrow T X$ and $\beta: Y \multimap \rightarrow=Y \rightarrow T Y$ and a Set-map $j: X \rightarrow Y$ which is a coalgebra homomomorphism (or, equivalently, $j^{\sharp} \cdot \alpha=\beta \cdot j^{\sharp}$ in $\mathcal{K} l(T)$ ) we have: $\alpha^{\omega}=\beta^{\omega} \cdot j^{\sharp}=\beta^{\omega} \circ j$. This property known as uniformity of $(-)^{\omega}$ w.r.t. the base maps (see e.g. [SP00]). In our setting, the fixpoint operator $(-)^{\omega}$ is uniform w.r.t. the base maps since the order of $\mathcal{K} l(T)$ is pointwise induced and since $\alpha^{\omega}=\bigwedge_{\kappa \in \text { Ord }}(x \mapsto x \cdot \alpha)^{\kappa}(\top)$ (see Remark 2.9).

This completes the proof of the lemma.

We are now ready to proceed with the proof of Theorem 5.11.

Proof. (Theorem 5.11) We have

$$
\omega \mathfrak{R a t}(\mathcal{A}) \supseteq \omega \mathfrak{R e g}(\mathcal{A})
$$

as it is enough to note that

$$
\|\alpha, \mathfrak{F}\|_{\omega} \cdot i_{n}=\left(\mathfrak{f}_{\mathfrak{F}} \cdot \alpha^{+}\right)^{\omega} \cdot i_{n}=\left(\mathfrak{f}_{\mathfrak{F}} \cdot \alpha^{+}\right)^{\omega} \cdot \mathfrak{f}_{\mathfrak{F}} \cdot \alpha^{+} \cdot i_{n}
$$

and take $r=\mathfrak{f}_{\mathfrak{F}} \cdot \alpha^{+} \cdot i_{n}, r_{k}=\mathfrak{f}_{\mathfrak{F}} \cdot \alpha^{+} \cdot k_{n}$.

Conversely, let $r, r_{i} \in \mathfrak{R e g}(1, m)$. Put $s=\left[r_{1}, \ldots, r_{m}\right]$ and consider any regular morphism $s^{\prime} \in \mathfrak{R e g}(m, m)$ such that $r=s^{\prime} \cdot 1_{m}$. For sake of clarity of notation let $m_{1} \triangleq m$ and $m_{2} \triangleq m$. Consider the map $\gamma \triangleq \sigma \cdot\left(s^{\prime}+s\right)$, where $\sigma: m+m \rightarrow m+m$ injects the first component of the coproduct into the second one and is the identity everywhere else. The morphism $\gamma$ is depicted below:

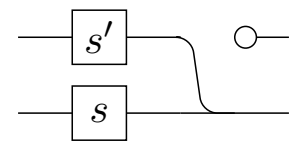

Note that this map is a regular morphism, so the map $\gamma^{\omega} \cdot 1_{m_{1}+m_{2}}$ is $\omega$-regular. Moreover, we get:

$$
\gamma^{\omega} \cdot 1_{m_{1}+m_{2}}=\gamma^{\omega} \cdot \gamma \cdot 1_{m_{1}+m_{2}}=\gamma^{\omega} \cdot \mathrm{in}^{m_{2}} \cdot s^{\prime} \cdot 1_{m_{1}}=\left[r_{1}, \ldots, r_{m}\right]^{\omega} \cdot r .
$$

This completes the proof.

\section{Probabilistic automata}

The main purpose of this section is to put probabilistic systems [BH97, SdVW09, Sok11, USH16, BG05, BGB12] into the framework of Section 5. Here, we focus our attention on probabilistic automata which are akin to fully probabilistic systems from Example 2.2[BH97, BMP15, BEMC00, Sok11]. 
6.1. Preliminaries. A probabilistic automaton is a tuple

$$
(X, \Sigma, P: X \times \Sigma \times X \rightarrow[0,1], \mathfrak{F}),
$$

where $X$ is a set of states, $\Sigma$ an alphabet, $P$ is a probability transition function, i.e. a function such that for any $x \in X$ we have $\sum_{(a, y) \in \Sigma \times X} P(x, a, y)=1$, and $\mathfrak{F} \subseteq X$ the set of accepting states.

For $C \subseteq X$ we define $P(x, a, C) \triangleq \sum_{y \in C} P(x, a, y)$. An execution fragment is a finite sequence $\mathfrak{s}=x_{0} \stackrel{a_{0}}{\rightarrow} x_{1} \stackrel{a_{1}}{\rightarrow} x_{2} \ldots x_{n-1} \stackrel{a_{n-1}}{\rightarrow} x_{n}$ such that $P\left(x_{i}, a_{i}, x_{i+1}\right)>0$. We define $\operatorname{first}(\mathfrak{s})=x_{0}, \operatorname{last}(\mathfrak{s})=x_{n}, \operatorname{length}(\mathfrak{s})=n, \operatorname{trace}(\mathfrak{s})=a_{0} \ldots a_{n-1}$ and $P(\mathfrak{s})=$ $\prod_{i=0, \ldots, n-1} P\left(x_{i}, a_{i}, x_{i+1}\right)$. An execution is an infinite sequence $\mathfrak{p}=x_{0} \stackrel{a_{0}}{\rightarrow} x_{1} \stackrel{a_{f}}{\rightarrow} x_{2} \stackrel{a_{2}}{\rightarrow} \ldots$ with $P\left(x_{i}, a_{i}, x_{i+1}\right)>0$.

Let $\operatorname{first}(\mathfrak{p}) \triangleq x_{0}, \operatorname{trace}(\mathfrak{p}) \triangleq a_{0} a_{1} \ldots, \mathfrak{p}^{(n)} \triangleq x_{0} \stackrel{a_{0}}{\rightarrow} \ldots \stackrel{a_{n}-1}{\rightarrow} x_{n}$ and $\mathfrak{p}_{n} \triangleq x_{n}$. For an execution fragment $\mathfrak{s}$ of length $n$ let $\mathfrak{s} \uparrow$ denote the set of all executions $\mathfrak{p}$ such that $\mathfrak{p}^{(n)}=\mathfrak{s}$.

Let $\operatorname{Exec}(x)$ denote the set of all executions $\mathfrak{p}$ such that $\operatorname{first}(\mathfrak{p})=x$. Let $\Sigma(x)$ be the smallest sigma field on $\operatorname{Exec}(x)$ which contains all sets $\mathfrak{s} \uparrow$ for any execution fragment $\mathfrak{s}$ with $\operatorname{first}(\mathfrak{s})=x$. Finally, let $\mathcal{Q}_{x}$ denote the unique probability measure on $\Sigma(x)$ such that $\mathcal{Q}_{x}(\mathfrak{s} \uparrow)=P(\mathfrak{s})$ for any execution fragment $\mathfrak{s}$ with $\operatorname{first}(\mathfrak{s})=x$. We will often drop the subscript and write $\mathcal{Q}$ instead of $\mathcal{Q}_{x}$ if the measure can be deduced from the context.

For $\Lambda \subseteq \Sigma^{*}$ and $C \subseteq X$ define $\operatorname{Exec}(\Lambda, C)$ to be the set of all executions $\mathfrak{p}=x_{0} \stackrel{a_{0}}{\rightarrow} x_{1} \ldots$ for which there is $n$ with $\operatorname{trace}\left(\mathfrak{p}^{(n)}\right) \in \Lambda$ and $x_{n} \in C$ and consider $\operatorname{Exec}(x, \Lambda, C) \triangleq$ $\operatorname{Exec}(\Lambda, C) \cap \operatorname{Exec}(x)$. As stated in [BH97] the set $\operatorname{Exec}(x, \Lambda, C)$ is $\Sigma(x)$-measurable. Additionally, put $\operatorname{Exec}(\Lambda) \triangleq \operatorname{Exec}(\Lambda, X)$ and $\operatorname{Exec}(x, \Lambda) \triangleq \operatorname{Exec}(x, \Lambda, X)$.

An execution $\mathfrak{p}=x=x_{0} \stackrel{a_{0}}{\rightarrow} x_{1} \stackrel{a_{1}}{\rightarrow} \ldots$ starting at $x$ is called $C$-accepting provided that it visists $C$ infinitely often, i.e. the set $\left\{i<\omega \mid x_{i} \in C\right\}$ is infinite. Let $\operatorname{AccExec}(C)$ denote all $C$-accepting executions and let $\operatorname{Acc} \operatorname{Exec}(x, C)=\operatorname{AccExec}(C) \cap \operatorname{Exec}(x)$. The set $\operatorname{AccExec}(x, C)$ is $\Sigma(x)$-measurable as

$$
\begin{aligned}
& \operatorname{AccExec}(x, C)=\bigcap_{n \geq 0} \bigcup_{k \geq n}\left\{\mathfrak{p} \in \operatorname{Exec}(x) \mid \mathfrak{p}_{k} \in C\right\}= \\
& \bigcap_{n \geq 0} \bigcup_{k \geq n} \operatorname{Exec}\left(x,\left\{\sigma \in \Sigma^{*} \mid \text { length of } \sigma=k\right\}, C\right)= \\
& \bigcap_{n \geq 0} \operatorname{Exec}\left(x,\left\{\sigma \in \Sigma^{*} \mid \text { length of } \sigma \geq n\right\}, C\right) .
\end{aligned}
$$

A curious reader is referred to e.g. [BEMC00, BH97] for more details on fully probabilistic systems and probability measures they induce.

Example 6.1. Consider the automaton $\left(\left\{s_{0}, s_{1}\right\}, \Sigma=\{0,1\}, P, \mathfrak{F}=\left\{s_{1}\right\}\right)$, where $P$ is the probability transition function given by the diagram below:

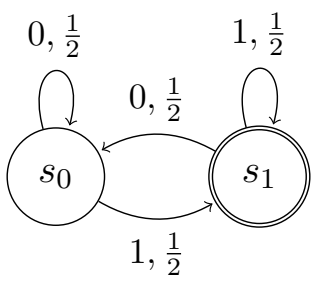


Let $\Lambda \subseteq \Sigma^{*}$. Then $\operatorname{Exec}\left(s_{0}, \Lambda, \mathfrak{F}\right)$ consists of all executions $\mathfrak{p}$ such that $\mathfrak{p}=x_{0} \stackrel{a_{0}}{\rightarrow} x_{1} \stackrel{a_{7}}{\rightarrow} x_{2} \stackrel{a_{2}}{\rightarrow}$ $\ldots$ for which $x_{0}=s_{0}$,

$$
\left(x_{i}, a_{i}, x_{i+1}\right) \text { is from }\left\{\left(s_{0}, 0, s_{0}\right),\left(s_{0}, 1, s_{1}\right),\left(s_{1}, 0, s_{0}\right),\left(s_{1}, 1, s_{1}\right)\right\},
$$

and such that there is $n$ with $a_{0} \ldots a_{n} \in \Lambda$ and $a_{n}=1$. Similarily, $\operatorname{Exec}\left(s_{1}, \Lambda, \mathfrak{F}\right)$ contains all executions $\mathfrak{p}$ such that $\operatorname{first}(\mathfrak{p})=s_{1}$, satisfying 6.1 and whose $\operatorname{trace}\left(\mathfrak{p}^{(n)}\right) \in \Lambda$ with its last letter equal to 1 for some $n \in \mathbb{N}$. In particular this means that $\mathcal{Q}\left(\operatorname{Exec}\left(s_{i},\{w 0\}, \mathfrak{F}\right)\right)=0$ and $\mathcal{Q}\left(\operatorname{Exec}\left(s_{i},\{w 1\}, \mathfrak{F}\right)\right)=\left(\frac{1}{2}\right)^{n+1}$ for any $w \in \Sigma^{*}$ whose length equals to $n$.

Now, $\operatorname{AccExec}\left(s_{0}, \mathfrak{F}\right)$ is the set of all executions $\mathfrak{p}=s_{0}=x_{0} \stackrel{a_{0}}{\rightarrow} x_{1} \stackrel{a_{1}}{\rightarrow} x_{2} \stackrel{a_{2}}{\rightarrow} \ldots$ such that it satisfies 6.1 and for any $n \in \mathbb{N}$ there is $i_{n} \geq n$ such that $\left(x_{i_{n}}, a_{i_{n}}, x_{i_{n}+1}\right)$ equals to $\left(s_{i}, 1, s_{1}\right)$. Similarily, $\operatorname{AccExec}\left(s_{1}, \mathfrak{F}\right)$ is the set of all executions whose first state is $s_{1}$ which satisfy the same argument as above. It is easy to see that $\mathcal{Q}\left(\operatorname{AccExec}\left(s_{0}, \mathfrak{F}\right)\right)=\mathcal{Q}\left(\operatorname{AccExec}\left(s_{1}, \mathfrak{F}\right)\right)=$ $1^{12}$.

The remaining part of this section will focus on finding a suitable setting in which we can model probabilistic automata and their (in)finite behaviour using the framework presented in the previous section.

Remark 6.2. Any probabilistic automaton can be modelled coalgebraically as a pair $(\alpha: X \rightarrow \mathcal{D}(\Sigma \times X), \mathfrak{F} \subseteq X)$, where $\mathcal{D}$ is the subdistribution monad from Example 2.2 and $\alpha$ is given by (see e.g. [SdVW09, BMSZ15]):

$$
\alpha(x)(a, y) \triangleq P(x, a, y) .
$$

The Kleisli category associated with the monad $\mathcal{D}\left(\Sigma^{*} \times \mathcal{I} d+\Sigma^{\omega}\right)^{13}$ is order enriched with the hom-set ordering given for $f, g: X \rightarrow \mathcal{D}\left(\Sigma^{*} \times Y+\Sigma^{\omega}\right)$ by:

$$
f \leq g \Longleftrightarrow f(x)(y) \leq g(x)(y) .
$$

Although the Kleisli category for this monad is $\omega$ Cpo-enriched, its hom-posets do not admit arbitrary finite suprema [HJS07, BMP15]. In other words, the setting is incompatible with the setting from the previous section. Hence, the remaining part of this section is focused on solving this issue based on ideas given in [GP14, BMP15].

6.2. Choosing the right monad. Here, we introduce a monad which is a suitable replacement for $\mathcal{D}$, i.e. it satisfies the desired properties to make it suitable for modeling (in)finite behaviours of probabilistic automata. Inspired by [GP14] we consider the continuous continuation monad parametrized by the set $[0,1]$ whose functorial part is defined for any set $X$ by:

$$
\mathfrak{D} X \triangleq(X \rightarrow[0,1]) \rightarrow_{\omega}[0,1]
$$

where $P \rightarrow \omega Q$ denotes the set of functions between two $\omega$ Cpo's $P$ and $Q$ which preserve suprema of $\omega$-chains. The identity maps and the composition in the Kliesli category for $\mathfrak{D}$

\footnotetext{
${ }^{12}$ This follows by the fact that any execution $\mathfrak{p}=x_{0} \stackrel{a_{0}}{\rightarrow} x_{1} \stackrel{a_{1}}{\rightarrow} \ldots$ satisfying 6.1 that is not a member of $\operatorname{AccExec}\left(s_{i}, \mathfrak{F}\right)$ satisfies the following condition: there is a natural number $n$ such that for all $i \geq n$ we have $x_{i}=s_{0}$ and $a_{i}=0$. Hence, the probability of the set of all such executions equals 0 .

${ }^{13}$ By applying the construction from Section 4 to $T=\mathcal{D}$ and $F=\Sigma \times \mathcal{I} d$ we obtain a monadic structure on the aforementioned functor.
} 
are as follows:

$$
\begin{aligned}
& \text { id }: X \rightarrow(X \rightarrow[0,1]) \rightarrow_{\omega}[0,1] ; x \mapsto \Delta_{x}, \text { where } \Delta_{x}(d) \triangleq d(x) . \\
& g \cdot f: X \rightarrow(Z \rightarrow[0,1]) \rightarrow_{\omega}[0,1], \text { where }(g \cdot f)(x)(d) \triangleq f(x)(y \mapsto g(y)(d))
\end{aligned}
$$

with $f: X \rightarrow(Y \rightarrow[0,1]) \rightarrow_{\omega}[0,1]$ and $g: Y \rightarrow(Z \rightarrow[0,1]) \rightarrow_{\omega}[0,1]$. Note that any $d: X \rightarrow[0,1] \in \mathcal{D} X$ can be assigned a function $\nu_{X}(d):(X \rightarrow[0,1]) \rightarrow_{\omega}[0,1]$ which maps any $d^{\prime}: X \rightarrow[0,1]$ onto

$$
\nu_{X}(d)\left(d^{\prime}\right)=\sum_{x \in X} d^{\prime}(x) \cdot d(x)
$$

It is not hard to see that this turns the family $\left\{\nu_{X}: \mathcal{D} X \rightarrow \mathfrak{D} X\right\}$ into a natural transformation $\nu: \mathcal{D} \Longrightarrow \mathfrak{D}$ which is a monad morphism between the monads $\mathcal{D}$ and $\mathfrak{D}$. Additionally, it is easy to see that there is a natural ordering of arrows in $\mathcal{K} l(\mathfrak{D})$ which share the same domain and codomain. Indeed, for $f, g: X \rightarrow \mathfrak{D} Y$ we have:

$$
f \leq g \Longleftrightarrow f(x)(d) \leq g(x)(d) \text { for any } x \in X \text { and } d: Y \rightarrow[0,1] .
$$

This turns the Kleisli category for the monad $\mathfrak{D}$ into an order enriched category. Moreover, the following theorem is true.

Lemma 6.3. The order enrichment of $\mathcal{K} l(\mathfrak{D})$ is pointwise induced, every hom-set of $\mathcal{K} l(\mathfrak{D})$ is a complete lattice and the category is $\omega \mathrm{Cpo-enriched} \mathrm{and} \mathrm{left} \mathrm{distributive.}$

Proof. The fact that the partial order is a complete lattice order is a direct corollary from the definition of $\mathfrak{D}$. We will now prove that Kleisli category for $\mathfrak{D}$ is $\omega C$ po-enriched. Take any ascending chain $\left\{f_{i}: X \rightarrow \mathfrak{D} Y\right\}_{i<\omega}$ of morphisms and note that

$$
\begin{aligned}
& {\left[g \cdot\left(\bigvee_{i} f_{i}\right)\right](x)(d)=\left(\bigvee_{i} f_{i}\right)(x)(y \mapsto g(y)(d))=} \\
& \bigvee_{i} f_{i}(x)(y \mapsto g(y)(d))=\bigvee_{i} g \cdot f_{i}(x)(d) \text { and } \\
& {\left[\left(\bigvee_{i} f_{i}\right) \cdot h\right](z)(d)=h(z)\left(x \mapsto \bigvee_{i} f_{i}(x)(d)\right) \stackrel{\dagger}{=}} \\
& \bigvee_{i} h(z)\left(x \mapsto f_{i}(x)(d)\right)=\bigvee_{i}\left(h \cdot f_{i}\right)(z)(d),
\end{aligned}
$$

where the equality marked with $(\dagger)$ follows from the fact that $\mathfrak{D} X$ consists of functions $(X \rightarrow[0,1]) \rightarrow \omega[0,1]$ that preserve $\omega$-chains. Note that the identities 6.3 and 6.4 hold more generally for an arbitrary family of morphisms $\left\{f_{i}\right\}_{i \in I}: X \rightarrow \mathfrak{D} Y$ and $g: Y \rightarrow \mathfrak{D} Z$. This shows left distributivity of the Kliesli category and ends the proof.

From the above it follows that $\mathcal{K} l(\mathfrak{D})$ satisfies $(\mathrm{A})$ - (D) from Section 5.

6.2.1. The monad for probabilistic automata and their behaviours. By instantiating the construction from Section 4 for $T=\mathfrak{D}$ and $F=\Sigma \times \mathcal{I} d$ we obtain the monad $\mathfrak{D}\left(\Sigma^{*} \times\right.$ $\left.\mathcal{I} d+\Sigma^{\omega}\right)$. We explicitly spell out the formula for the identity maps and the composition in 
$\mathcal{K} l\left(\mathfrak{D}\left(\Sigma^{*} \times \mathcal{I} d+\Sigma^{\omega}\right)\right)$ as it will be used throughout the remaining part of this section. For any set $X$ the identity map id is given by:

$$
\begin{aligned}
& \text { id }: X \rightarrow\left(\Sigma^{*} \times X+\Sigma^{\omega} \rightarrow[0,1]\right) \rightarrow \omega[0,1], \\
& \operatorname{id}(x)(d)=d(\varepsilon, x) .
\end{aligned}
$$

Moreover, for $f: X \rightarrow \mathfrak{D}\left(\Sigma^{*} \times Y+\Sigma^{\omega}\right)$ and $g: Y \rightarrow \mathfrak{D}\left(\Sigma^{*} \times Z+\Sigma^{\omega}\right)$ the map $g \cdot f: X \rightarrow$ $\mathfrak{D}\left(\Sigma^{*} \times Z+\Sigma^{\omega}\right)$ is:

$$
\begin{aligned}
& g \cdot f: X \rightarrow\left(\Sigma^{*} \times Z+\Sigma^{\omega} \rightarrow[0,1]\right) \rightarrow_{\omega}[0,1], \\
& (g \cdot f)(x)(d)=f(x)\left((\sigma, y) \mapsto g(y)\left(d_{\mid \sigma}\right) \text { and } v \mapsto d(v)\right),
\end{aligned}
$$

where for $d: \Sigma^{*} \times Z+\Sigma^{\omega} \rightarrow[0,1]$ and $\sigma \in \Sigma^{*}$ the map $d_{\mid \sigma}: \Sigma^{*} \times Z+\Sigma^{\omega} \rightarrow[0,1]$ is given by $d_{\mid \sigma}(\tau, z)=d(\sigma \tau, z)$ for $\tau \in \Sigma^{*}, z \in Z$ and $d_{\mid \sigma}(v)=d(\sigma v)$ for $v \in \Sigma^{\omega}$.

The following statement is a direct consequence of the definition of the monad $\mathfrak{D}\left(\Sigma^{*} \times\right.$ $\left.\mathcal{I} d+\Sigma^{\omega}\right)$ and the properties of $\mathfrak{D}$ from the previous subsection.

Theorem 6.4. $\mathcal{K} l\left(\mathfrak{D}\left(\Sigma^{*} \times \mathcal{I} d+\Sigma^{\omega}\right)\right)$ satisfies $(A)$ - (D) from Section 5 .

6.3. (In)finite behaviour. Let $(\alpha, \mathfrak{F})$ be a pair as in Remark 6.2 and consider

$$
\left(\widehat{\alpha}: X \stackrel{\alpha}{\rightarrow} \mathcal{D}(\Sigma \times X) \stackrel{\nu_{\Sigma \times X}}{\rightarrow} \mathfrak{D}(\Sigma \times X) \hookrightarrow \mathfrak{D}\left(\Sigma^{*} \times X+\Sigma^{\omega}\right), \mathfrak{F}\right),
$$

where $\nu$ is given by 6.2 . We see that the map $\widehat{\alpha}$ can be viewed as an endomorphism in the Kleisli category for the monad $\mathfrak{D}\left(\Sigma^{*} \times \mathcal{I} d+\Sigma^{\omega}\right)$.

The rest of this section is devoted to presenting some properties of $\|\widehat{\alpha}, \mathfrak{F}\|$ and $\|\widehat{\alpha}, \mathfrak{F}\|_{\omega}$ for the pair $(\widehat{\alpha}, \mathfrak{F})$. We show that the values $\|\widehat{\alpha}, \mathfrak{F}\|(x)$ and $\|\widehat{\alpha}, \mathfrak{F}\|_{\omega}(x)$ of behaviour functions encode probabilities of certain events from $\Sigma(x)$. The main results are summarized in Theorem 6.6 and 6.7 .

6.3.1. Finite behaviour. At first let us focus on describing the finite behaviour map $\|\widehat{\alpha}, \mathfrak{F}\|$. Before we do that (see Theorem 6.6 for details) we need to present some intermediate results first. The following lemma is a direct consequence of the definition of $\widehat{\alpha}^{*}, \widehat{\alpha}^{+}$and the composition in $\mathcal{K} l\left(\mathfrak{D}\left(\Sigma^{*} \times \mathcal{I} d+\Sigma^{\omega}\right)\right)$.

Lemma 6.5. We have:

$$
\begin{aligned}
& \widehat{\alpha}^{+}, \widehat{\alpha}^{*}: X \rightarrow\left(\Sigma^{*} \times X+\Sigma^{\omega} \rightarrow[0,1]\right) \rightarrow_{\omega}[0,1] \\
& \widehat{\alpha}^{+}(x)(d)=\sum_{(a, y) \in \Sigma \times X} \widehat{\alpha}^{*}(y)\left(d_{\mid a}\right) \cdot \alpha(x)(a, y) \\
& \widehat{\alpha}^{*}(x)(d)=\max \left\{d(\varepsilon, x), \sum_{(a, y) \in \Sigma \times X} \widehat{\alpha}^{*}(y)\left(d_{\mid a}\right) \cdot \alpha(x)(a, y)\right\} .
\end{aligned}
$$

Now, consider a subset $\mathfrak{F} \subseteq X$ and note that, in the setting of this subsection, the map $\mathfrak{f}_{\mathfrak{F}}$ is explicitly given by:

$$
\begin{aligned}
& \mathfrak{f}_{\mathfrak{F}}: X \rightarrow\left(\Sigma^{*} \times X+\Sigma^{\omega} \rightarrow[0,1]\right) \rightarrow \omega[0,1], \\
& \mathfrak{f}_{\mathfrak{F}}(x)(d)=\left\{\begin{array}{cl}
d(\varepsilon, x) & \text { for } x \in \mathfrak{F}, \\
0 & \text { otherwise. }
\end{array}\right.
\end{aligned}
$$


Hence, the map $! \cdot \mathfrak{f}_{\mathfrak{F}}: X \rightarrow\left(\Sigma^{*} \times 1+\Sigma^{\omega} \rightarrow[0,1]\right) \rightarrow_{\omega}[0,1]$ is given by:

$$
\left(! \cdot \mathfrak{f}_{\mathfrak{F}}\right)(x)(d)=\left\{\begin{array}{cl}
d(\varepsilon, 1) & \text { for } x \in \mathfrak{F} \\
0 & \text { otherwise }
\end{array}\right.
$$

Therefore, the finite behaviour of $(\widehat{\alpha}, \mathfrak{F})$ is:

$$
\begin{aligned}
& \|\widehat{\alpha}, \mathfrak{F}\|: X \rightarrow\left(\Sigma^{*} \times 1+\Sigma^{\omega} \rightarrow[0,1]\right) \rightarrow \omega[0,1] \\
& \|\widehat{\alpha}, \mathfrak{F}\|(x)(d)=\left(! \cdot \mathfrak{f}_{\mathfrak{F}} \cdot \widehat{\alpha}^{*}\right)(x)(d)= \\
& \widehat{\alpha}^{*}(x)\left((\sigma, y) \mapsto\left\{\begin{array}{cc}
d(\sigma, 1) & y \in \mathfrak{F}, \\
0 & \text { otherwise }
\end{array} \text { and } v \mapsto 0\right) .\right.
\end{aligned}
$$

For $\Lambda \subseteq \Sigma^{*}$ and $\sigma \in \Sigma$ put $\Lambda / \sigma \triangleq\{\tau \mid \sigma \tau \in \Lambda\}$ and consider a mapping $\chi_{\Lambda}: \Sigma^{*} \times 1+\Sigma^{\omega} \rightarrow$ $[0,1]$ given by:

$$
\chi_{\Lambda}(x)=\left\{\begin{array}{cc}
1 & x=(\sigma, 1) \text { and } \sigma \in \Lambda \\
0 & \text { otherwise. }
\end{array}\right.
$$

If we define a function $\chi_{\mathfrak{F}, \Lambda}: \Sigma^{*} \times X+\Sigma^{\omega} \rightarrow[0,1]$ by:

$$
\chi_{\mathfrak{F}, \Lambda}(\tau, y)=\left\{\begin{array}{cc}
1 & y \in \mathfrak{F} \text { and } \tau \in \Lambda, \\
0 & \text { otherwise }
\end{array} \text { and } \chi_{\mathfrak{F}, \Lambda}(v)=0 .\right.
$$

then $\|\widehat{\alpha}, \mathfrak{F}\|(x)\left(\chi_{\Lambda}\right)=\widehat{\alpha}^{*}(x)\left(\chi_{\mathfrak{F}, \Lambda}\right)$ and:

$$
\|\widehat{\alpha}, \mathfrak{F}\|(x)\left(\chi_{\Lambda}\right)=\left\{\begin{array}{cc}
1 & \varepsilon \in \Lambda \text { and } x \in \mathfrak{F} \\
\sum_{(a, y) \in \Sigma \times X} \widehat{\alpha}^{*}(y)\left(\chi_{\mathfrak{F}, \Lambda / a}\right) \cdot \alpha(x)(a, y) & \text { otherwise }
\end{array}\right.
$$

A careful analysis of the formulae from [BH97, BMP15] describing the value $\mathcal{Q}(\operatorname{Exec}(x, \Lambda, C))$ and the above observations lead us to the statement below. It turns out that for any state $x$ the value $\|\widehat{\alpha}, \mathfrak{F}\|(x)\left(\chi_{\Lambda}\right)$ is the probability of reaching a state in $\mathfrak{F}$ from $x$ via an execution fragment whose trace is a member of $\Lambda$ :

Theorem 6.6. We have:

$$
\|\widehat{\alpha}, \mathfrak{F}\|(x)\left(\chi_{\Lambda}\right)=\mathcal{Q}(\operatorname{Exec}(x, \Lambda, \mathfrak{F}))
$$

6.3.2. Infinite behaviour. Let us focus on the infinite behaviour of $(\widehat{\alpha}, \mathfrak{F})$ introduced in the previous section given by

$$
\|\widehat{\alpha}, \mathfrak{F}\|_{\omega}=\left(\mathfrak{f}_{\mathfrak{F}} \cdot \widehat{\alpha}^{+}\right)^{\omega}: X \rightarrow \mathfrak{D}\left(\Sigma^{\omega}\right) .
$$

The following theorem gives us insight into what (some of) the values of the infinite behaviour function are. To be more precise, we show that for a state $x$ and a subset $\Lambda \subseteq \Sigma^{*}$, the value of the infinite behaviour of $x$ calculated for the characteristic function of the set of all infinite sequences from $\Sigma^{\omega}$ with prefixes in $\Lambda$ equals to the probability of $\mathfrak{F}$-accepting executions starting at $x$ whose trace prefix belongs to $\Lambda$.

Theorem 6.7. For any $\Lambda \subseteq \Sigma^{*}$ we have:

$$
\begin{aligned}
& \|\widehat{\alpha}, \mathfrak{F}\|_{\omega}: X \rightarrow\left(\Sigma^{\omega} \rightarrow[0,1]\right) \rightarrow_{\omega}[0,1], \\
& \|\widehat{\alpha}, \mathfrak{F}\|_{\omega}(x)\left(\chi_{\Lambda \cdot \Sigma^{\omega}}\right)=\mathcal{Q}(\operatorname{Exec}(x, \Lambda) \cap \operatorname{AccExec}(x, \mathfrak{F})),
\end{aligned}
$$

where $\Lambda \cdot \Sigma^{\omega} \triangleq\left\{w v \in \Sigma^{\omega} \mid w \in \Lambda\right.$ and $\left.v \in \Sigma^{\omega}\right\}$. 
Proof. The proof is divided into three parts.

Part 1. We will first show the statement holds for $\Lambda=\{\varepsilon\}$, i.e. we prove that

$$
\|\widehat{\alpha}, \mathfrak{F}\|_{\omega}(x)\left(\chi_{\Sigma^{\omega}}\right)=\mathcal{Q}(\operatorname{AccExec}(x, \mathfrak{F}))
$$

Indeed, since

$$
\operatorname{AccExec}(x, \mathfrak{F})=\bigcap_{n \geq 0} \bigcup_{k \geq n}\left\{\mathfrak{p} \in \operatorname{Exec}(x) \mid \mathfrak{p}_{k} \in \mathfrak{F}\right\}
$$

we have:

$$
\mathcal{Q}(\operatorname{AccExec}(x, \mathfrak{F}))=\lim _{n \rightarrow \infty} \mathcal{Q}\left(A_{n}^{x}\right),
$$

where $A_{n}^{x} \triangleq \bigcup_{k \geq n}\left\{\mathfrak{p} \in \operatorname{Exec}(x) \mid \mathfrak{p}_{k} \in \mathfrak{F}\right\}=\operatorname{Exec}\left(x,\left\{\sigma \in \Sigma^{*} \mid\right.\right.$ length of $\left.\left.\sigma \geq n\right\}, \mathfrak{F}\right)$ is a descending chain of $\Sigma(x)$-measurable sets. Let us now consider a family of maps $\left\{G_{n}: X \rightarrow\left(\Sigma^{\omega} \rightarrow[0,1]\right) \rightarrow_{\omega}[0,1]\right\}_{n}$ defined inductively as follows:

$$
G_{0}(x)(d)=1 \text { and } G_{n+1}=G_{n} \cdot \mathfrak{f}_{\mathfrak{F}} \cdot \widehat{\alpha}^{+} .
$$

Note that the sequence $\left\{G_{n}(x)(d)\right\}_{n}$ is descending for any fixed $x \in X$ and $d$ and that

$$
\|\widehat{\alpha}, \mathfrak{F}\|_{\omega}(x)(d)=\lim _{n \rightarrow \infty} G_{n}(x)(d) .
$$

We will now show that $G_{n}(x)\left(\chi_{\Sigma^{\omega}}\right)=\mathcal{Q}\left(A_{n}^{x}\right)$ for $n \geq 1$. For $G_{1}$ we have:

$$
\begin{aligned}
& G_{1}(x)\left(\chi_{\Sigma^{\omega}}\right)=\left(G_{0} \cdot \mathfrak{f}_{\mathfrak{F}} \cdot \widehat{\alpha}^{+}\right)(x)\left(\chi_{\Sigma^{\omega}}\right)= \\
& \widehat{\alpha}^{+}(x)\left((\sigma, y) \mapsto G_{0} \cdot \mathfrak{f}_{\mathfrak{F}}(y)\left(\chi_{\Sigma^{\omega}} \mid \sigma\right) \text { and } v \mapsto \chi_{\Sigma^{\omega}}(v)\right)= \\
& \widehat{\alpha}^{+}(x)\left((\sigma, y) \mapsto G_{0} \cdot \mathfrak{f}_{\mathfrak{F}}(y)\left(\chi_{\Sigma^{\omega}}\right) \text { and } v \mapsto \chi_{\Sigma^{\omega}}(v)\right) .
\end{aligned}
$$

In the above

$$
\begin{aligned}
& G_{0} \cdot \mathfrak{f}_{\mathfrak{F}}(y)\left(\chi_{\Sigma^{\omega}}\right)=\mathfrak{f}_{\mathfrak{F}}(y)\left((\tau, z) \mapsto G_{0}(z)\left(\chi_{\Sigma^{\omega} \mid \tau}\right) \text { and } v \mapsto \chi_{\Sigma^{\omega}}(v)\right)= \\
& \mathfrak{f}_{\mathfrak{F}}(y)((\tau, z) \mapsto 1 \text { and } v \mapsto 1)=\left\{\begin{array}{cc}
1 & y \in \mathfrak{F} \\
0 & \text { otherwise. }
\end{array}\right.
\end{aligned}
$$

Hence, if we continue with 6.13 we get:

$$
\widehat{\alpha}^{+}(x)\left((\sigma, y) \mapsto\left\{\begin{array}{cc}
1 & y \in \mathfrak{F} \\
0 & \text { otherwise }
\end{array} \text { and } v \mapsto 1\right)=\mathcal{Q}\left(A_{1}^{x}\right)\right.
$$

If we now assume by induction that the statement holds for some $n>1$ then

$$
G_{n} \cdot \mathfrak{f}_{\mathfrak{F}}(y)\left(\chi_{\Sigma^{\omega} \omega}\right)=\mathfrak{f}_{\mathfrak{F}}(y)\left((\tau, z) \mapsto \mathcal{Q}\left(A_{n}^{z}\right) \text { and } v \mapsto 1\right)=\left\{\begin{array}{cc}
\mathcal{Q}\left(A_{n}^{y}\right) & y \in \mathfrak{F} \\
0 & \text { otherwise. }
\end{array}\right.
$$

Hence, by following a similar reasoning to the one applied to $G_{1}$ we get:

$$
G_{n+1}(x)\left(\chi_{\Sigma^{\omega}}\right)=\widehat{\alpha}^{+}\left((\sigma, y) \mapsto\left\{\begin{array}{cc}
\mathcal{Q}\left(A_{n}^{y}\right) & y \in \mathfrak{F} \\
0 & \text { otherwise }
\end{array} \quad \text { and } v \mapsto 1\right)=\mathcal{Q}\left(A_{n+1}^{x}\right)\right.
$$

Part 2. We will now show that the following holds:

$$
\|\widehat{\alpha}, \mathfrak{F}\|_{\omega}(x)\left(\chi_{\left\{a_{0} a_{1} \ldots a_{n}\right\} \cdot \Sigma^{\omega}}\right)=\mathcal{Q}\left(\operatorname{Exec}\left(x,\left\{a_{0} \ldots a_{n}\right\}\right) \cap \operatorname{Acc} \operatorname{Exec}(x, \mathfrak{F})\right) .
$$

Assume $\mathfrak{s}=x=x_{0} \stackrel{a_{0}}{\rightarrow} \ldots \stackrel{a_{n-1}}{\rightarrow} x_{n} \stackrel{a_{n}}{\rightarrow} x_{n+1}=x^{\prime}$. Then for $\sigma \in \Sigma^{*}$ of length less than or equal to $n$ we have $\chi_{\left\{a_{0} a_{1} \ldots a_{n}\right\} \cdot \Sigma^{\omega} \mid \sigma}$ is equal $\chi_{\left\{a_{k} \ldots a_{n}\right\} \cdot \Sigma^{\omega}}$ if $\sigma=a_{0} \ldots a_{k-1}$ and it is the constantly 
equal to zero function otherwise. This observation together with the fact that

$$
\begin{aligned}
& \mathcal{Q}(\mathfrak{s} \uparrow \cap \operatorname{AccExec}(x, \mathfrak{F}))=\mathcal{Q}(\operatorname{AccExec}(x, \mathfrak{F}) \mid \mathfrak{s} \uparrow) \cdot Q(\mathfrak{s} \uparrow)= \\
& \mathcal{Q}\left(\operatorname{AccExec}\left(x^{\prime}, \mathfrak{F}\right)\right) \cdot \mathcal{Q}(\mathfrak{s} \uparrow)
\end{aligned}
$$

and induction allows us to prove the assertion.

Part 3. The statement from Part 2. can be easily generalized to

$$
\|\widehat{\alpha}, \mathfrak{F}\|_{\omega}(x)\left(\chi_{\left\{w_{1}\right\} \cdot \Sigma^{\omega} \cup\left\{w_{2}\right\} \cdot \Sigma^{\omega}}\right)=\mathcal{Q}\left(\operatorname{Exec}\left(x,\left\{w_{1}, w_{2}\right\}\right) \cap \operatorname{AccExec}(x, \mathfrak{F})\right),
$$

for any pair of incomparable words $w_{1}, w_{2} \in \Sigma^{*}$ with respect to lexicographic ordering on $\Sigma^{*}$. Indeed, in this case the sets $\left\{w_{1}\right\} \cdot \Sigma^{\omega}$ and $\left\{w_{2}\right\} \cdot \Sigma^{\omega}$ are disjoint. If $\sigma$ is not comparable with $w_{1}$ and $w_{2}$ then $\chi_{\left\{w_{1}\right\} \cdot \Sigma^{\omega} \cup\left\{w_{2}\right\} \cdot \Sigma^{\omega} \mid \sigma}$ is constantly equal to zero function. However, if $\sigma$ is comparable wit $w_{i}$ then for all such $\sigma$ which are sufficiently long we have: $\chi_{\left\{w_{1}\right\} \cdot \Sigma^{\omega} \cup\left\{w_{2}\right\} \cdot \Sigma^{\omega} \mid \sigma}=\chi_{\left\{w_{i}\right\} \cdot \Sigma^{\omega} \mid \sigma}$. Hence, by the same argument as before we prove the desired assertion. Note that the equation generalizes to any finite set of incomparable words $\left\{w_{1}, \ldots, w_{n}\right\}$.

Finally, the general statement holds since the function

$$
\|\widehat{\alpha}, \mathfrak{F}\|_{\omega}(x):\left(\Sigma^{\omega} \rightarrow[0,1]\right) \rightarrow_{\omega}[0,1]
$$

preserves suprema of $\omega$-chains. Indeed, let $\Lambda \subseteq \Sigma^{*}$ and note that $\Lambda \cdot \Sigma^{\omega}=\Lambda^{\prime} \cdot \Sigma^{\omega}$ for a countable subset $\Lambda^{\prime} \subseteq \Lambda$ of incomparable words $\Lambda^{\prime}=\left\{w_{1}, w_{2}, \ldots\right\}$. Then

$$
\chi_{\Lambda \cdot \Sigma^{*}}=\chi_{\Lambda^{\prime} \cdot \Sigma^{\omega}}=\bigvee_{n} \chi_{\left\{w_{1}, \ldots, w_{n}\right\} \cdot \Sigma^{\omega}}
$$

Hence,

$$
\begin{aligned}
& \|\widehat{\alpha}, \mathfrak{F}\|_{\omega}(x)\left(\chi_{\Lambda \cdot \Sigma^{\omega}}\right)=\|\widehat{\alpha}, \mathfrak{F}\|_{\omega}(x)\left(\chi_{\Lambda^{\prime} \cdot \Sigma^{\omega}}\right)=\|\widehat{\alpha}, \mathfrak{F}\|_{\omega}(x)\left(\bigvee_{n} \chi_{\left\{w_{1}, \ldots, w_{n}\right\} \cdot \Sigma^{\omega}}\right)= \\
& \bigvee_{n}\|\widehat{\alpha}, \mathfrak{F}\|_{\omega}(x)\left(\chi_{\left\{w_{1}, \ldots, w_{n}\right\} \cdot \Sigma^{\omega}}\right)=\bigvee_{n} \mathcal{Q}\left(\operatorname{Exec}\left(x,\left\{w_{1}, \ldots, w_{n}\right\}\right) \cap \operatorname{AccExec}(x, \mathfrak{F})\right)= \\
& \mathcal{Q}\left(\operatorname{Exec}\left(x, \Lambda^{\prime}\right) \cap \operatorname{AccExec}(x, \mathfrak{F})\right)=\mathcal{Q}(\operatorname{Exec}(x, \Lambda) \cap \operatorname{AccExec}(x, \mathfrak{F})) .
\end{aligned}
$$

Example 6.8. Let us consider the probabilistic automaton from Example 6.1 and put it into the framework of the Lawvere theory for the monad $\mathfrak{D}\left(\Sigma^{*} \times \mathcal{I} d+\Sigma^{\omega}\right)$. Below, we calculate (some values of) finite and infinite behaviours of the automaton derived from Example 6.1 in a direct manner (i.e. without applying Theorem 6.6 or Theorem 6.7). By following the guidelines of Remark 6.2 we obtain $(\alpha, \mathfrak{F})$, where $\alpha: X \rightarrow \mathcal{D}(\Sigma \times X)$ given by $\alpha\left(s_{0}\right)=P\left(s_{0},-,-\right)$ and $\alpha\left(s_{1}\right)=P\left(s_{1},-,-\right)$. Moreover, $\widehat{\alpha}: X \rightarrow \mathfrak{D}\left(\Sigma^{*} \times X+\Sigma^{\omega}\right)$ is defined by

$$
\widehat{\alpha}\left(s_{0}\right):\left(\Sigma^{*} \times\left\{s_{0}, s_{1}\right\}+\Sigma^{\omega} \rightarrow[0,1]\right) \rightarrow_{\omega}[0,1] ; d \mapsto \frac{1}{2} \cdot d\left(0, s_{0}\right)+\frac{1}{2} \cdot d\left(1, s_{1}\right)
$$


and $\widehat{\alpha}\left(s_{1}\right)=\widehat{\alpha}\left(s_{0}\right)$. Next, observe that by Lemma 6.5 the morphisms $\widehat{\alpha}^{+}$and $\widehat{\alpha}^{*}$ are the least solutions to:

$$
\begin{aligned}
& \widehat{\alpha}^{+}\left(s_{0}\right)(d)=\widehat{\alpha}^{+}\left(s_{1}\right)(d)=\frac{1}{2} \cdot \widehat{\alpha}^{*}\left(s_{0}\right)\left(d_{\mid 0}\right)+\frac{1}{2} \cdot \widehat{\alpha}^{*}\left(s_{1}\right)\left(d_{\mid 1}\right) \\
& \widehat{\alpha}^{*}\left(s_{0}\right)(d)=\max \left\{d\left(\varepsilon, s_{0}\right), \frac{1}{2} \cdot \widehat{\alpha}^{*}\left(s_{0}\right)\left(d_{\mid 0}\right)+\frac{1}{2} \cdot \widehat{\alpha}^{*}\left(s_{1}\right)\left(d_{\mid 1}\right)\right\} \\
& \widehat{\alpha}^{*}\left(s_{1}\right)(d)=\max \left\{d\left(\varepsilon, s_{1}\right), \frac{1}{2} \cdot \widehat{\alpha}^{*}\left(s_{0}\right)\left(d_{\mid 0}\right)+\frac{1}{2} \cdot \widehat{\alpha}^{*}\left(s_{1}\right)\left(d_{\mid 1}\right)\right\} .
\end{aligned}
$$

Consider $w=a_{0} a_{1} \ldots a_{n} \in \Sigma^{*}, \chi_{\{w 0\}}, \chi_{\{w 1\}}$ as in 6.7 and $\chi_{\mathfrak{F},\{w 0\}}, \chi_{\mathfrak{F},\{w 1\}}$ as in 6.8. Then by 6.9

$$
\|(\widehat{\alpha}, \mathfrak{F})\|\left(s_{0}\right)\left(\chi_{\{w 0\}}\right)=\frac{1}{2} \cdot \widehat{\alpha}^{*}\left(s_{0}\right)\left(\chi_{\mathfrak{F},\{w 0\}_{/ 0}}\right)+\frac{1}{2} \cdot \widehat{\alpha}^{*}\left(s_{1}\right)\left(\chi_{\mathfrak{F},\{w 0\} / 1}\right)
$$

By carefully analysing the formula for $\widehat{\alpha}^{*}$ we conclude that, in our case,

$$
\|\widehat{\alpha}, \mathfrak{F}\|\left(s_{0}\right)\left(\chi_{\{w 0\}}\right)=0 .
$$

Similarily, by 6.9 we have

$$
\|\widehat{\alpha}, \mathfrak{F}\|\left(s_{0}\right)\left(\chi_{\{w 1\}}\right)=\frac{1}{2} \cdot \widehat{\alpha}^{*}\left(s_{0}\right)\left(\chi_{\mathfrak{F},\{w 1\}_{/ 0}}\right)+\frac{1}{2} \cdot \widehat{\alpha}^{*}\left(s_{1}\right)\left(\chi_{\mathfrak{F},\{w 1\}_{/ 1}}\right) .
$$

In this case, however, a thorough analysis of the formula for $\widehat{\alpha}^{*}$ leads us to the following conclusion:

$$
\|\widehat{\alpha}, \mathfrak{F}\|\left(s_{0}\right)\left(\chi_{\{w 1\}}\right)=\left(\frac{1}{2}\right)^{n+1}
$$

Now, in order to compute $\|\widehat{\alpha}, \mathfrak{F}\|_{\omega}$ first consider $\mathfrak{f}_{\mathfrak{F}} \cdot \widehat{\alpha}^{+}$which is given by:

$$
\mathfrak{f}_{\mathfrak{F}} \cdot \widehat{\alpha}^{+}\left(s_{i}\right)(d)=\widehat{\alpha}^{+}\left(s_{i}\right)\left(\left(\sigma, s_{0}\right) \mapsto 0 \text { and }\left(\sigma, s_{1}\right) \mapsto d\left(\sigma, s_{1}\right) \text { and } v \mapsto d(v)\right) \text {. }
$$

The morphism $\|\widehat{\alpha}, \mathfrak{F}\|_{\omega}=\left(\mathfrak{f}_{\mathfrak{F}} \cdot \widehat{\alpha}^{+}\right)^{\omega}: X \rightarrow\left(\Sigma^{\omega} \rightarrow[0,1]\right) \rightarrow_{\omega}[0,1]$ is the greatest map satisfying $\left(\mathfrak{f}_{\mathfrak{F}} \cdot \widehat{\alpha}^{+}\right)^{\omega}=\left(\mathfrak{f}_{\mathfrak{F}} \cdot \widehat{\alpha}^{+}\right)^{\omega} \cdot \mathfrak{f}_{\mathfrak{F}} \cdot \widehat{\alpha}^{+}$. In particular, this means that

$$
\begin{aligned}
& \left(\mathfrak{f}_{\mathfrak{F}} \cdot \widehat{\alpha}^{+}\right)^{\omega}\left(s_{i}\right)(d)=\left(\mathfrak{f}_{\mathfrak{F}} \cdot \widehat{\alpha}^{+}\right)^{\omega} \cdot \mathfrak{f}_{\mathfrak{F}} \cdot \widehat{\alpha}^{+}\left(s_{i}\right)(d)= \\
& \left(\mathfrak{f}_{\mathfrak{F}} \cdot \widehat{\alpha}^{+}\right)\left(s_{i}\right)\left(\left(\sigma, s_{j}\right) \mapsto\left(\mathfrak{f}_{\mathfrak{F}} \cdot \widehat{\alpha}^{+}\right)^{\omega}\left(s_{j}\right)\left(d_{\mid \sigma}\right) \text { and } v \mapsto d(v)\right)
\end{aligned}
$$

For $d=\chi_{\Sigma} \omega$ we have $d_{\mid \sigma}=\chi_{\Sigma^{\omega}}$ and hence $\left(\mathfrak{f}_{\mathfrak{F}} \cdot \widehat{\alpha}^{+}\right)^{\omega}$ solves to the following equation for $x$ :

$$
x\left(s_{i}\right)\left(\chi_{\Sigma^{\omega}}\right)=\left(\mathfrak{f}_{\mathfrak{F}} \cdot \widehat{\alpha}^{+}\right)\left(s_{i}\right)\left(\left(\sigma, s_{j}\right) \mapsto x\left(s_{j}\right)\left(\chi_{\Sigma^{\omega}}\right) \text { and } v \mapsto 1\right) .
$$

In order to compute $\left(\mathfrak{f}_{\mathfrak{F}} \cdot \widehat{\alpha}^{+}\right)^{\omega}\left(s_{i}\right)\left(\chi_{\Sigma^{\omega}}\right)$ take $d^{\prime}: \Sigma^{*} \times X+\Sigma^{\omega} \rightarrow[0,1]$ given by $\left(\sigma, s_{0}\right) \mapsto$ 0 and $\left(\sigma, s_{1}\right) \mapsto 1$ and $v \mapsto 1$ and note that it satisfies $d_{\mid \sigma}^{\prime}=d^{\prime}$. Moreover, it is not hard to see that $\widehat{\alpha}^{*}\left(s_{i}\right)\left(d^{\prime}\right)=1^{14}$. Therefore, the following equation holds:

$$
\begin{aligned}
& 1=\frac{1}{2} \cdot 1+\frac{1}{2} \cdot 1= \\
& \frac{1}{2} \cdot \widehat{\alpha}^{*}\left(s_{0}\right)\left(d^{\prime}\right)+\frac{1}{2} \cdot \widehat{\alpha}^{*}\left(s_{1}\right)\left(d^{\prime}\right)=\widehat{\alpha}^{+}\left(s_{i}\right)\left(d^{\prime}\right)= \\
& \left(\mathfrak{f}_{\mathfrak{F}} \cdot \widehat{\alpha}^{+}\right)\left(s_{i}\right)\left(\left(\sigma, s_{j}\right) \mapsto 1 \text { and } v \mapsto 1\right) .
\end{aligned}
$$

This proves that if we put

$$
\left(\mathfrak{f}_{\mathfrak{F}} \cdot \widehat{\alpha}^{+}\right)^{\omega}\left(s_{i}\right)\left(\chi_{\Sigma} \omega\right)=1
$$

\footnotetext{
${ }^{14}$ Indeed, $\widehat{\alpha}^{*}\left(s_{1}\right)\left(d^{\prime}\right)=1$ follows trivially from the fact that $d^{\prime}\left(\varepsilon, s_{1}\right)=1$. By the recursive formula describing $\widehat{\alpha}^{*}$ and by induction we show that $\widehat{\alpha}^{*}\left(s_{0}\right)\left(d^{\prime}\right) \geq \frac{1}{2}+\frac{1}{4}+\cdots+\frac{1}{2^{n}}$ for any $n \in \mathbb{N}$. This proves the assertion.
} 
then it satisfies 6.14. Thus,

$$
\|\widehat{\alpha}, \mathfrak{F}\|_{\omega}\left(s_{i}\right)\left(\chi_{\Sigma^{\omega}}\right)=1 .
$$

Remark 6.9. By Theorem 6.4 probabilistic automata can be put into the framework of Section 5. Hence, Kleene theorems hold for any suitable choice of $\mathcal{A}$. In particular, we may take $\mathcal{A}$ to be the least set of maps containing all morphisms of the form

$$
n \stackrel{\alpha}{\rightarrow} \mathcal{D}(\Sigma \times n) \stackrel{\nu_{\Sigma \times n}}{\rightarrow} \mathfrak{D}(\Sigma \times n) \hookrightarrow \mathfrak{D}\left(\Sigma^{*} \times n+\Sigma^{\omega}\right)
$$

and satisfying the properties listed in the beginning of Subsection 5.3.

6.4. Summary. The purpose of this section was to put probabilistic automata into a monadic framework from Section 5 and reason about their (in)finite behaviours. We achieved this by introducing the continuous continuation monad $\mathfrak{D}$ and viewing probabilistic automata transition maps as coalgebras

$$
X \rightarrow \mathfrak{D}\left(\Sigma^{*} \times X+\Sigma^{\omega}\right) .
$$

The monad $\mathfrak{D}\left(\Sigma^{*} \times \mathcal{I} d+\Sigma^{\omega}\right)$ gives rise to a Kleisli category which satisfies (A) - (D) from Section 5 making it possible to consider finite and infinite behaviours of automata taken into consideration. We proved that the behaviour maps encode probabilities of certain events from the execution space. These probabilities were attained without changing the underlying category: the type monad and the automata taken into consideration are Set-based. Additionally, Theorem 6.7 suggests that our infinite behaviour with BAC for probabilistic automata is similar to the one presented in [USH16]. However, in loc. cit. the base category for probabilistic systems was the category of measurable spaces and measurable functions. Hence, by Remark 6.9, Kleene theorems can be instantiated in our setting directly, but it is not possible to do so in the setting from [USH16].

\section{Summary}

The purpose of this paper was to develop a coalgebraic (categorical) framework to reason about abstract automata and their finite and infinite behaviours satisfying BAC. We achieved this goal by constructing a monad suitable for handling the types of behaviours we were interested in and defining them in the right setting. A natural and direct consequence of this treatment was Theorem 5.10 and Theorem 5.11, i.e. a (co)algebraic characterization of regular and $\omega$-regular behaviour for systems whose type is a Set-based monad satisfying some additional properties. Our theory of finite and infinite behaviour for abstract automata has been successfully instantiated on: non-deterministic automata, tree automata and probabilistic automata.

Future work. Given our natural characterization of coalgebraic $(\omega$-)regular languages we ask if it is possible to characterize it in terms of a preimage of a subset of a finite algebraic structure. Especially, considering the fact that by Theorem 5.4 the pair of hom-sets $(\mathbb{T}(n, n), \mathbb{T}(n, 0))$ equipped with suitable operations resembles a Wilke algebra used in the algebraic characterization of these languages (see e.g. [PP04] for details).

Our definitions of the operators $(-)^{*}$ and $(-)^{\omega}$ via the least and greatest fixpoints suggest a connection between our line of work and $\mu$-calculus [Koz83, BdRV01, Ven20]. In particular, it would be interesting to clarify how our coalgebraic framework fits into the framework of 
coalgebraic modal $\mu$-calculus and its semantics (e.g. [CKP11, FLV10]) with an emphasis laid on non-classical systems, e.g. probabilistic systems from Section 6.

Related work. The first coalgebraic take on $\omega$-languages was presented in [CV12], where authors put deterministic Muller automata with Muller acceptance condition into a coalgebraic framework. Our work is related to a more recent paper [USH16], where Urabe et al. give a coalgebraic framework for modelling behaviour with Büchi acceptance condition for $(T, F)$ systems. The main ingredient of their work is a solution to a system of equations which uses least and greatest fixpoints. This is done akin to Park's [Par81] classical characterization of $\omega$-languages via a system of equations. In our paper we also use least and greatest fixpoints, however, the operators we consider are the two natural types of operators $(-)^{*}=\mu x$.id $\vee x \cdot(-)$ and $(-)^{\omega}=\nu x . x \cdot(-)$ which generalize the language operators $(-)^{*}$ and $(-)^{\omega}$ known from the classical theory of regular and $\omega$-regular languages. The definitions of behaviours of an automaton are presented in terms of simple expressions involving Kleisli composition and the above operators. This allows us to state and prove generic Kleene theorems for $(\omega$-)regular input which was not achieved in [USH16] and (in our opinion) would be difficult to obtain in that setting. To summarize, the major differences between our work and [USH16] are the following:

- we use the setting of systems with internal moves (i.e. coalgebras over a monad) to discuss infinite behaviour with BAC, which is given in terms of a simple expression using $(-)^{*}$ and $(-)^{\omega}$ in the Kleisli category,

- we provide the definition of (in)finite behaviours of a system and build a bridge between regular and $\omega$-regular behaviours by characterizing them on a categorical level in terms of the Kleene theorems.

Abstract finite automata have already been considered in the computer science literature in the context of Lawvere iteration theories with analogues of Kleene theorems stated and proven (see e.g. [Ési97, ÉK11, ÉK13, EH09, BE93]). Some of these results seem to be presented using a slightly different language than ours (see Theorem 5.10 and e.g. [BE93, Theorem 1.4]). We decided to state Theorem 5.10 the way we did, in order to make a direct generalization of the classical Kleene theorem for regular input and to give a coalgebraic interpretation which is missing in [Ési97, ÉK11, ÉK13, EH09, BE93]. We should also mention that the infinite behaviour with BAC was defined in loc. cit. only for a very specific type of theories (i.e. the matricial theories over an algebra with an infinite iteration operator), which do not encompass e.g. non-deterministic Büchi tree automata and their infinite tree languages or probabilistic automata and their infinite behaviour.

\section{REFERENCES}

[AHM14] Jirí Adámek, Mahdieh Haddadi, and Stefan Milius. Corecursive algebras, corecursive monads and bloom monads. Logical Methods in Computer Science, 10(3), 2014.

[BdRV01] Patrick Blackburn, Maarten de Rijke, and Yde Venema. Modal Logic, volume 53 of Cambridge Tracts in Theoretical Computer Science. Cambridge University Press, 2001.

[BE93] Stephen Bloom and Zoltán Ésik. Iteration Theories. The Equational Logic of Iterative Processes. Monographs in Theoretical Computer Science. Springer, 1993.

[Bec69] Jon Beck. Distributive laws. In B. Eckmann, editor, Seminar on Triples and Categorical Homology Theory, pages 119-140, Berlin, Heidelberg, 1969. Springer Berlin Heidelberg.

[BEMC00] Christel Baier, Bettina Engelen, and Mila E. Majster-Cederbaum. Deciding bisimilarity and similarity for probabilistic processes. J. Comput. Syst. Sci., 60(1):187-231, 2000. 
[BG05] Christel Baier and Marcus Grosser. Recognizing omega-regular languages with probabilistic automata. In Proceedings of the 20th Annual IEEE Symposium on Logic in Computer Science, LICS '05, pages 137-146, Washington, DC, USA, 2005. IEEE Computer Society.

[BGB12] Christel Baier, Marcus Grösser, and Nathalie Bertrand. Probabilistic omega-automata. J. ACM, 59(1):1:1-1:52, March 2012.

[BH97] Christel Baier and Holger Hermanns. Weak bisimulation for fully probabilistic processes. In $C A V$, pages 119-130, 1997.

[BMP15] Tomasz Brengos, Marino Miculan, and Marco Peressotti. Behavioural equivalences for coalgebras with unobservable moves. Journal of Logical and Algebraic Methods in Programming, 84(6):826$852,2015$.

[BMSZ15] Filippo Bonchi, Stefan Milius, Alexandra Silva, and Fabio Zanasi. Killing epsilons with a dagger: A coalgebraic study of systems with algebraic label structure. Theoretical Computer Science, 604:102-126, 2015.

[BP16] Tomasz Brengos and Marco Peressotti. A Uniform Framework for Timed Automata. In Josée Desharnais and Radha Jagadeesan, editors, 27th International Conference on Concurrency Theory (CONCUR 2016), volume 59 of Leibniz International Proceedings in Informatics (LIPIcs), pages 26:1-26:15, Dagstuhl, Germany, 2016. Schloss Dagstuhl-Leibniz-Zentrum fuer Informatik.

[BP19] Tomasz Brengos and Marco Peressotti. Behavioural equivalences for timed systems. Logical Methods in Computer Science, 15(1), 2019.

[Bre14] Tomasz Brengos. On coalgebras with internal moves. In Marcello M. Bonsangue, editor, Proc. CMCS, Lecture Notes in Computer Science, pages 75-97. Springer, 2014.

[Bre15] Tomasz Brengos. Weak bisimulation for coalgebras over order enriched monads. Logical Methods in Computer Science, 11(2):1-44, 2015.

[Bre18] Tomasz Brengos. A Coalgebraic Take on Regular and omega-Regular Behaviour for Systems with Internal Moves. In Sven Schewe and Lijun Zhang, editors, 29th International Conference on Concurrency Theory (CONCUR 2018), volume 118 of Leibniz International Proceedings in Informatics (LIPICs), pages 25:1-25:18, Dagstuhl, Germany, 2018. Schloss Dagstuhl-LeibnizZentrum fuer Informatik.

[Büc90] J. Richard Büchi. On a Decision Method in Restricted Second Order Arithmetic, pages 425-435. Springer New York, New York, NY, 1990.

[BW02] Michael Barr and Charles Wells. Toposes, Triples and Theories. Springer, 2002.

[Cîr10] Corina Cîrstea. Generic infinite traces and path-based coalgebraic temporal logics. Electr. Notes Theor. Comput. Sci., 264(2):83-103, 2010.

[CKP11] Corina Cîrstea, Clemens Kupke, and Dirk Pattinson. EXPTIME tableaux for the coalgebraic mu-calculus. Log. Methods Comput. Sci., 7(3), 2011.

[CV12] Vincenzo Ciancia and Yde Venema. Stream automata are coalgebras. In Proc. CMCS, volume 7399 of Lecture Notes in Computer Science, pages 90-108, 2012.

[EH09] Zoltán Ésik and Tamás Hajgató. Iteration grove theories with applications. In Proc. Algebraic Informatics, volume 5725 of Lecture Notes in Computer Science, pages 227-249. Springer, 2009.

[ÉK11] Zoltán Ésik and Werner Kuich. A Unifying Kleene Theorem for Weighted Finite Automata, pages 76-89. Springer Berlin Heidelberg, Berlin, Heidelberg, 2011.

[ÉK13] Zoltán Ésik and Werner Kuich. Modern Automata Theory, page 222. 2013.

[Ési97] Zoltán Ésik. Completeness of park induction. Theor. Comput. Sci., 177(1):217-283, 1997.

[FLV10] Gaëlle Fontaine, Raul Andres Leal, and Yde Venema. Automata for coalgebras: An approach using predicate liftings. In Samson Abramsky, Cyril Gavoille, Claude Kirchner, Friedhelm Meyer auf der Heide, and Paul G. Spirakis, editors, Automata, Languages and Programming, 37th International Colloquium, ICALP 2010, Bordeaux, France, July 6-10, 2010, Proceedings, Part II, volume 6199 of Lecture Notes in Computer Science, pages 381-392. Springer, 2010.

[FS18] Brendan Fong and David I Spivak. Seven Sketches in Compositionality: An Invitation to Applied Category Theory. arxiv:1803.05316, 2018.

[GP14] Sergey Goncharov and Dirk Pattinson. Coalgebraic weak bisimulation from recursive equations over monads. In Javier Esparza, Pierre Fraigniaud, Thore Husfeldt, and Elias Koutsoupias, editors, Proc. ICALP, volume 8573 of Lecture Notes in Computer Science, pages 196-207. Springer, 2014. 
[GTW02] Erich Grädel, Wolfgang Thomas, and Thomas Wilke, editors. Automata Logics, and Infinite Games: A Guide to Current Research, page 392. Springer-Verlag New York, Inc., New York, NY, USA, 2002.

[Gum99] H. Peter Gumm. Elements of the general theory of coalgebras. LUATCS 99, Rand Afrikaans University, 1999.

[Has06] Ichiro Hasuo. Generic forward and backward simulations. In Prof. CONCUR, volume 4137 of Lecture Notes in Computer Science, pages 406-420, 2006.

[HJS07] Ichiro Hasuo, Bart Jacobs, and Ana Sokolova. Generic trace semantics via coinduction. Logical Methods in Computer Science, 3(4), 2007.

[HMRU00] John E. Hopcroft, Rajeev Motwani, Rotwani, and Jeffrey D. Ullman. Introduction to Automata Theory, Languages and Computability. Addison-Wesley Longman Publishing Co., Inc., Boston, MA, USA, 2nd edition, 2000.

[HP07] Martin Hyland and John Power. The category theoretic understanding of universal algebra: Lawvere theories and monads. Electronic Notes in Theoretical Computer Science, 172:437-458, 2007.

[Jac04] Bart Jacobs. Trace semantics for coalgebras. Electr. Notes Theor. Comput. Sci., 106:167-184, 2004.

[JSS12] Bart Jacobs, Alexandra Silva, and Ana Sokolova. Trace semantics via determinization. In Proc. CMCS, volume 7399 of Lecture Notes in Computer Science, pages 109-129, 2012.

[Kle56] S. C. Kleene. Representation of events in nerve nets and finite automata. In Claude Shannon and John McCarthy, editors, Automata Studies, pages 3-41. Princeton University Press, Princeton, NJ, 1956.

[KN01] Bakhadyr Khoussainov and Anil Nerode. Automata Theory and Its Applications. Birkhauser Boston, Inc., USA, 2001.

[Koc72] Anders Kock. Strong functors and monoidal monads. Archiv der Mathematik, 23(1):113-120, 1972.

[Koz83] Dexter Kozen. Results on the propositional $\mu$-calculus. Theoretical Computer Science, 27:333-354, 1983.

[Law63] F. W. Lawvere. Functorial semantics of algebraic theories. Proc. Nat. Acad. Sci. U.S.A., 50:869$872,1963$.

[Mil89] Robin Milner. Communication and Concurrency. Prentice-Hall, 1989.

[ML78] Saunders Mac Lane. Categories for the Working Mathematician, volume 5 of Graduate Texts in Mathematics. Springer-Verlang New York, 1978.

[Mul93] Philip S. Mulry. Lifting theorems for kleisli categories. In Stephen D. Brookes, Michael G. Main, Austin Melton, Michael W. Mislove, and David A. Schmidt, editors, Mathematical Foundations of Programming Semantics, $9^{\text {th }}$ International Conference, New Orleans, LA, USA, April 7-10, 1993, Proceedings, volume 802 of Lecture Notes in Computer Science, pages 304-319. Springer, 1993.

[Par81] David Park. Concurrency and automata on infinite sequences. In Peter Deussen, editor, Theoretical Computer Science, pages 167-183, Berlin, Heidelberg, 1981. Springer Berlin Heidelberg.

[PP04] Jean-Eric Pin and Dominique Perrin. Infinite Words: Automata, Semigroups, Logic and Games, page 538. Elsevier, 2004.

[Rut00] Jan J. M. M. Rutten. Universal coalgebra: a theory of systems. Theoretical Computer Science, 249(1):3-80, 2000.

[San11] Davide Sangiorgi. Introduction to Bisimulation and Coinduction. Cambridge University Press, 2011.

[SdVW09] A. Sokolova, E. P. de Vink, and H. Woracek. Coalgebraic weak bisimulation for action-type systems. Scientific Annals of Computer Science, 19:93-144, 2009.

[Sel11] Peter Selinger. A survey of graphical languages for monoidal categories. In New Structures for Physics, volume 813, pages 289-355, 2011.

[Sok11] Ana Sokolova. Probabilistic systems coalgebraically: A survey. Theoretical Computer Science, 412(38):5095-5110, 2011.

[SP00] Alex Simpson and Gordon Plotkin. Complete axioms for categorical fixed-point operators. In Proceedings of the 15th Annual IEEE Symposium on Logic in Computer Science, LICS '00, pages 30-, Washington, DC, USA, 2000. IEEE Computer Society. 
[SW13] Alexandra Silva and Bram Westerbaan. A coalgebraic view of $\epsilon$-transitions. In Reiko Heckel and Stefan Milius, editors, Proc. CALCO, volume 8089 of Lecture Notes in Computer Science, pages 267-281. Springer, 2013.

[UH15] Natsuki Urabe and Ichiro Hasuo. Coalgebraic infinite traces and kleisli simulations. CoRR, abs/1505.06819, 2015 .

[USH16] Natsuki Urabe, Shunsuke Shimizu, and Ichiro Hasuo. Coalgebraic Trace Semantics for Buechi and Parity Automata. In Josée Desharnais and Radha Jagadeesan, editors, 27th International Conference on Concurrency Theory (CONCUR 2016), volume 59 of Leibniz International Proceedings in Informatics (LIPICs), pages 24:1-24:15, Dagstuhl, Germany, 2016. Schloss Dagstuhl-LeibnizZentrum fuer Informatik.

[Ven20] Yde Venema. Lecutres on the modal $\mu$-calculus. 2020. 